\title{
Review of the Effect of Temperature on Oil-Water Relative Permeability in Porous Rocks of Oil Reservoirs
}

By

\section{Abstract:}

7 Thermal methods of heavy oil recovery involve multiphase flow at high temperatures. Numerical 8 simulation studies of such processes require accounting for changes in the multi-phase flow

9 behavior of the rock-fluid system with increasing temperature. Although the effect of temperature 10 on two-phase relative permeability has been studied for more than five decades, it remains an 11 unresolved issue. Experimental results that frequently contradict each other are still being reported 12 and the issue remains a matter of debate. The purpose of this review is to critically examine the 13 reported results and explore the possible reasons for contradictory results. We have examined the 14 reported results of more frequently cited papers from past five decades and attempted to rationalize 15 the disagreements in findings.

16 There appear to be three main reasons for the lack of consensus in experimentally observed results.

17 The measurements of relative permeability at high temperature are complex and the reported 18 results often include experimental artifacts. Secondly, meaningful relative permeability 19 measurements require that capillary forces control the fluid distribution within the pore space, but 20 this condition is difficult to ensure in viscous oil systems. The third reason is that the impact of 21 temperature is not same in all rock-fluid systems, it depends on how the wettability, interfacial 22 tension and the pore geometry changes with temperature.

23 It becomes apparent that it is not advisable to generalize the effect of temperature on relative 24 permeability from previous studies without having a good understanding of how the underlying 25 parameters that can influence the relative permeability are changing with temperature. The relative 26 permeability of a specific petroleum reservoir may (or may not) vary with temperature. 


\section{1. INTRODUCTION}

Thermal recovery of heavy oil and bitumen involves two-phase and three-phase flow of

3 oil, water and gas at high temperatures in oil bearing porous formations. Modeling of such

4 processes requires accounting for changes in the multiphase flow properties of reservoir rocks

5 resulting from the increase in temperature. Heating the rock from original reservoir temperature to

6 the high temperatures, which can exceed $300{ }^{\circ} \mathrm{C}$ in steam injection and much higher in in-situ

7 combustion processes [1], brings about changes in rock-fluid properties that can have a large

8 impact on the flow behavior. The viscosity of heavy oil decreases by several orders of magnitude

9 [2-7] and this by itself can significantly change the flow characteristics [8-11]. Furthermore, such

10 large increase in temperature can also change other rock-fluid properties, including wettability [4,

11 12-20], interfacial tension $[7,14,16,21-25]$ and pore geometry.

12 Multiphase flow in porous media is complicated due to contributions of many factors, such

13 as, complex pore geometry, the rock wettability, properties of different phases, capillary pressure,

14 pore and throat size distributions and compressibility of the porous medium. The commonly used

15 mathematical description of multiphase flow in porous media is based on the extension of the

16 Darcy's equation to multiphase flow [26] by introducing the concept of effective permeability for

17 each phase that varies with saturations of different phases. Under two-phase flow conditions, the

18 effective permeability for each fluid phase becomes a function of its own saturation [27-29]. This

19 dependence of effective permeability on saturation is usually described by defining a relative

20 permeability, which represents the ratio of the effective permeability to a base permeability, which

21 is often the absolute permeability of the medium [28-30]. The advantage of using relative

22 permeability to describe the variation with saturation is that it separates the changes in absolute

23 permeability from the effects of fluid saturation. It allows one to account for the effect of 
1 permeability heterogeneity in the reservoir by assuming that the same relative permeability curve

2 applies at different values of the absolute permeability. In most reservoir engineering flow studies,

3 the relative permeability is one of the most crucial parameters [31].

4 The knowledge of two-phase water/oil relative permeability is needed to predict the 5 production rate, breakthrough time and the ultimate oil recovery in processes involving 6 displacement of oil by water $[32,33]$. The relative permeability also affects the pressure response 7 and velocity profile of fluids flowing through the porous rock in such displacements. The relative 8 permeability varies from one oil reservoir to another and it may even be different for two core 9 plugs with the same geometry, geology, lithology, composition, and physical properties (porosity 10 and permeability) but with different pore size distributions [29, 34]. In the same rock, the relative 11 permeability can change with the type of fluids saturating the pores [29, 32]. Accordingly, there is 12 always some uncertainty when a given set of relative permeability data, which was measured using

13 the best available technique on a core sample from a specific reservoir using native fluids, is used 14 for analysis of other similar reservoirs [29, 30]. Actually, uncertainty remains, to some extent, even 15 in the analysis of the reservoir from which the core sample was obtained, due to the possibility of 16 changes in the behavior in different parts of the formation.

17 Numerous studies have been reported in the petroleum literature on relative permeability 18 properties of different types of porous media and on the effects of rock-fluid characteristics that 19 affect the flow behavior $[29,35,36]$. The effect of temperature on relative permeability curves has 20 received significant attention since 1950's [6]. There are published reports that contradict each 21 other on the temperature impact on two-phase relative permeability for various systems $[2-4,6,7$, $2212,25]$. In addition, numerous studies have attempted over the years to present the effect of 23 temperature on relative permeability by proposing some useful relative permeability models even 
1 for a particular system [5, 25, 37-42]. The objective of this study is to critically review such

2 published articles $[2-4,6-8,12,13,15-17,19,21,24,25,38,40-53]$ on the effect of temperature

3 on two-phase relative permeability and distill useful information and insights into the changes in

4 behavior that occur as a function of the temperature. This involves careful examination of the effect

5 of temperature on characteristics of relative permeability curves for different porous media types

6 and various fluid types in a wide range of temperature and pressure. This extensive survey

7 endeavors to clarify how the contribution of various variables including wettability alteration,

8 viscosity ratio, capillary end effect, saturation history, data interpretation method, type of oil and

9 porous medium, and the employed experimental procedure, as well as human errors and

10 experimental artifacts could have led to contradictory findings. In this review, the most cited

11 publications since 1956 are examined and the effect of temperature on different attributes of the

12 relative permeability curves are extracted and analyzed.

\section{2. RELATIVE PERMEABILITY CONCEPT}

14 When two immiscible fluids flow simultaneously through a reservoir rock, the conductivity

15 of the rock to each fluid depends not only on the permeability of the rock but also on the relative

16 amount of each fluid present in the pore space. In other words, the effective permeability to each

17 fluid depends on the absolute permeability of the rock and the fraction of the pore space occupied

18 by that fluid, which is called the fluid saturation. The relative permeability is defined as the

19 effective permeability divided by a base permeability, which is often the absolute permeability of

20 the medium, as shown in Eq. (1) below.

$$
k_{r i}\left(S_{i}\right)=k_{e i}\left(S_{i}\right) / k_{a b s}
$$


Where $k_{r i}$ is the relative permeability to fluid $i$, when its saturation is $S_{i}, k_{e i}\left(S_{i}\right)$ is the

2 effective permeability to fluid $i$ at the same saturation, $k_{a b s}$ is the absolute permeability and $i$

3 denotes either oil or water. Very often, under two-phase flow conditions in oil reservoirs, the

4 relative permeability to each fluid is a function only of the saturation of that fluid and it is

5 independent of other flow parameters. The rationale for treating the relative permeability to be a

6 determinable function of saturation is based on the concept that the two immiscible fluids flow

7 largely in parallel but separate pore networks and that the fluid distribution within the pores is

8 controlled primarily by capillary forces [27, 29]. It is generally true that the capillary forces acting

9 on the fluids under typical reservoir flow conditions are several orders of magnitude larger than

10 viscous and inertial forces $[54,55]$. Therefore, the distribution of the two immiscible fluids is often

11 controlled by the capillary forces $[54,55]$. This dominance of surface forces favors the fluid

12 distribution that minimizes the free energy of solid-fluid and fluid-fluid interfaces. Consequently,

13 the wetting phase and the non-wetting phase tend to follow predictable pore occupancy rules and

14 the distribution of phases within the pore space is theoretically predictable at any specific

15 saturation $[29,56]$. The relative permeability to each phase depends on which portion of the

16 available pore space it occupies and this, coupled with the capillarity controlled pore occupancy

17 rules, makes the relative permeability a determinable function of saturation. It should be

18 understood that any deviation from the capillarity controlled fluid distribution would result in the

19 relative permeability becoming dependent on other factors.

20 For quantifying the effect of various rock-fluid characteristics on two-phase oil/water

21 relative permeability curves, the two curves can be characterized with six different features. These

22 parameters include the irreducible water saturation, the residual oil saturation, oil relative

23 permeability at the irreducible water saturation, water relative permeability at the residual oil 
1 saturation and the shapes of the two curves (which can often be described by the degree of

2 curvature involved). Before discussing the effect of temperature on these six parameters of the

3 relative permeability curves, the significance of each of them is explained below.

\section{$4 \quad$ 2.1. Irreducible water and residual oil saturation}

5 The irreducible water saturation is simply defined as the lowest water saturation which can

6 be achieved by oil flooding a water saturated core plug [29]. At irreducible water saturation, the

7 relative permeability to water becomes 'practically' zero; as a result, no further water can be

8 displaced by continued injection of oil [29, 30, 36]. Similarly, the residual oil saturation is the

9 lowest oil saturation obtained by water flooding an oil-saturated porous medium [29]. Therefore,

10 the oil relative permeability during the water flooding declines gradually and becomes 'practically'

11 zero at the residual oil saturation [29, 30]. The word 'practically' is used here to emphasize the

12 fact that in experimental measurements of these parameters the value may not reach the

13 mathematical zero point but becomes small enough to be considered negligible $[8,16,51]$.

\section{2.2. Endpoint relative permeability to oil and water}

15 The endpoint relative permeability to water is the highest value of water relative

16 permeability and occurs at the residual oil saturation [29]. At this point, only water is able to flow

17 in the two-phase system $[29,56]$. In water-wet systems, the residual oil saturation will be present

18 as isolated oil droplets or blobs of varying size $[29,56]$. This discontinuous saturation of oil

19 remains trapped by capillary forces [56]. In oil-wet systems, the residual oil saturation can remain

20 continuous as thin wetting film on pore surfaces but it is practically immobile [56-59].

21 The endpoint relative permeability to oil occurs at the irreducible water saturation, i.e.

22 when only oil is able to flow in the two phase system [36]. In water-wet systems the irreducible 
1 water saturation may remain continuous in the form of a thin wetting film on pore surfaces but its

2 mobility is practically zero [57-60].

\section{2.3. Shapes of oil and water relative permeability curves}

The relative permeability of each phase is zero at its residual saturation and becomes

5 highest when its saturation reaches the maximum possible value $[29,30,56]$. In between these two

6 limits, the relative permeability is expected to increase monotonically with increasing phase

7 saturation, since more flow channels become available for its flow as it occupies more of the total

8 pore space [27]. The manner in which the relative permeability increases with increasing saturation

9 (i.e. the shape of relative permeability curve) depends on several factors including the pore size

10 distribution, pore connectivity, wettability and saturation history [27, 29, 56].

\section{2.4. Effect of Temperature}

Theoretically, the relative permeability of each phase depends on which portion of the total

13 pore space each fluid occupies and the conductivity of that portion of the pore space. Therefore,

14 the increase in temperature can affect the relative permeability only two ways: 1) it changes the

15 conductivity of the occupied pore space by altering the pore geometry or 2) it re-distributes the

16 two fluids within the pore space by changing the capillary and other forces that control the fluid

17 distribution. Although, it is possible that a large increase in the temperature can generate in-situ

18 thermal stresses that may significantly affect the pore geometry, the laboratory measurements of

19 relative permeability are generally conducted under conditions that minimize this possibility.

20 Hence, the observed effects of temperature on relative permeability arise mostly from re-

21 distribution of fluids due to altered balance between capillary and viscous forces. Consequently,

22 understanding the effect of temperature on relative permeability requires understanding how the 
1 forces that control fluid distribution within the pore space change with temperature. An additional

2 factor that needs to be considered is the impact of differences in the experimental protocol used

3 for evaluating the effect of temperature on relative permeability. These differences include the

4 selection of experimental technique, e.g. steady-state versus displacement method, the differences

5 in the experimental materials and conditions, and the way in which the selected measurement

6 technique was implemented.

7 In this paper, we first review the commonly used methods for measuring the relative

8 permeability and then, in Section 4, we examine the impact of the experimental conditions and

9 materials used in various reported studies. In Section 5, we review the changes in rock-fluid

10 properties with temperature that can affect the relative permeability. Finally, in section 6 , we

11 review the reported effects of temperature on oil-water relative permeability curves by examining

12 how the six parameters of oil-water relative permeability curves change with temperature and

13 attempt to explain the experimental observations in terms of the materials and methodology used

14 in different studies. Section 7 lists the conclusions of this study.

\section{3. METHODS FOR DETERMINATION OF RELATIVE PERMEABILITY CURVES}

16 The two-phase relative permeability of a porous medium can be evaluated using several

17 techniques, including different experimental measurement techniques, methods based on

18 mathematical modeling of two-phase flow, empirical correlations, and by the analogy method [27,

19 29, 30]. The laboratory methods include the steady-state and unsteady-state flow tests, the

20 centrifuge method and the use of capillary pressure measurements to estimate relative permeability

$21[27,29,30]$. The focus in this paper is on the laboratory measurements. 
The measurement of relative permeability generally involves the use of a small sample of

2 the porous medium and creation of one-dimensional two-phase flow in the sample, during which

3 the conductivity of the medium to each phase can be inferred from measured and imposed test

4 parameters. Four distinct experimental approaches have been mentioned in the literature and are

5 considered reasonably reliable.

\section{$6 \quad 3.1$. Steady-state approach}

The most direct, and for that reason also the most reliable, experimental method is the steady-state method [31, 61]. It involves injection of a fixed ratio of oil and water (or any two

9 immiscible phases) into the porous medium sample at a constant rate to generate one-dimensional

10 flow until the pressure drop across the sample and the fractional flow coming out of the sample

11 become stable $[29,36,62]$. Although the saturation profile within the sample and the pressure drop

12 across its length change in the early stage of the test, it is expected that eventually the saturation

13 profile and the pressure will become steady and the produced fractional flow will be identical to

14 the injected one $[6,7,18,24,29,36,61-63]$. To obtain the complete relative permeability curve,

15 usually between 5 and 10 different ratios of water/oil flow rates are employed [24, 63, 64]. Darcy's

16 law is applied to estimate the effective permeability of each phase using the measured pressure

17 drop across the core and imposed flow rates [29, 48, 62].

Numerous techniques have been reported to establish an uniform saturation profile, since

19 the capillary end effect, which causes the wetting-phase saturation to be high near the outlet end,

20 is always a big concern in such measurements [29]. Moreover, to obtain reliable results, the

21 average water and oil saturations have to be determined accurately, either by material balance or

22 using an in-situ measurement technique [36]. The reported steady-state relative permeability

23 techniques include: Penn-State method [65-67], Single-Sample dynamic method [68-70], 
1 Stationary fluid method [71], Hassler method [72], Hafford method [70], and Dispersed feed

2 method [70]. Among these methods, the Hassler method has been used more often, although it is

3 accepted that this technique only works properly when the sample is strongly wetted by one of the

4 fluids [29]. Experimental difficulties have been reported in using the Hassler procedure under

5 conditions of intermediate wettability[73, 74]. The general comments on pros and cons of different

6 method for measuring relative permeability are summarized in Table 1.

7 Table 1. General comments on laboratory relative permeability measurement methods [30].

\begin{tabular}{|c|c|}
\hline Steady-State approach & $\begin{array}{l}\text { - } \quad \text { Full saturation range can be covered } \\
\text { - } \quad \text { Relative permeability down to } 10^{-3} \\
\text { - } \quad \text { Lower accuracy near endpoint saturations } \\
\text { - } \quad \text { Very time consuming and expensive because of the required } \\
\text { pressure and saturation stabilization for each reading. }\end{array}$ \\
\hline Unsteady-State approach & $\begin{array}{l}\text { - } \text { Fast and less expensive, hence widely used } \\
\text { - Relative permeability down to } 10^{-3} \\
\text { - Representative of the dynamic reservoir situation } \\
\text { - A part of the saturation range not directly accessible } \\
\end{array}$ \\
\hline Centrifuge approach & $\begin{array}{l}\text { - } \text { Relative permeability only of displaced phase } \\
\text { - } \quad \text { Relative permeability down to } 10^{-6} \\
\text { - } \quad \text { Better estimation of endpoint region }\end{array}$ \\
\hline
\end{tabular}

\subsection{Unsteady-state approach}

The unsteady-state approach is a displacement method in which an immiscible displacing

10 fluid is injected at constant rate into a core sample, initially containing the highest possible

11 saturation of the displaced fluid (under two-phase conditions), and the transient behavior of

12 pressure drop across the length of the core and the volumes of produced phases are recorded

13 periodically $[31,36]$. The main advantage of this method is that it is less time-consuming [30].

14 The relative permeability characteristics are inferred from the recorded production and pressure

15 drop history of the displacement test [31]. This requires a more complex calculation procedure 
1 than the steady-state method [29]. Further comments about the pros and cons of unsteady-state

2 approach are included in Table 1.

\section{3.3. Capillary pressure approach}

The capillary pressure approach relates the relative permeability curves to measured

5 capillary pressure-saturation relationship [29]. The rationale behind it is that, when a porous

6 medium exhibits a clear wetting preference for one of the two fluid phases, the distribution of the

7 two fluids within the pore space is governed by clear-cut pore occupancy rules that result in the

8 wetting fluid occupying the smallest available pores. Since both the drainage capillary pressure

9 and the relative permeability at any given wetting phase saturation are controlled by the radius of

10 the smallest pore invaded by the non-wetting phase, it is logical that there is a relationship between

11 them. Several scholars [75-79] have developed analytical equations for estimating the non-wetting

12 and wetting phase relative permeability from capillary pressure data.

\section{3.4. Centrifuge approach}

14 Centrifuge method for relative permeability is similar to centrifuge method for measuring

15 capillary pressure except that now the volume of displaced fluid produced is recorded against time

16 after each step change in the rotational speed of the centrifuge. The fluid production is similar to

17 the unsteady state displacement with specified inlet and outlet pressures $[80,81]$ and a

18 computational method to obtain the relative permeability is available [81]. This technique does not

19 suffer from viscous fingering problems, which sometimes happens during unsteady-state tests [29,

2061 ]. Also, the centrifuge relative permeability test can be conducted substantially faster than the

21 steady-state method [29]. Table 1 includes some other comments on the pros and cons of this

22 method. 
1 4. IMPACT OF EXPERIMENTAL CONDITIONS ON OBSERVED EFFECT OF

2 TEMPERATURE ON RELATIVE PERMEABILITY

One of the important factors controlling the relative permeability is the wettability state of porous medium $[29,56]$. The properties of the two fluid phases used will affect the contact angle,

5 wettability, interfacial tension, and capillary pressure. Many other factors can also affect the

6 relative permeability to varying degrees. Some researchers $[2,15,17,20,22,25,42,45,50]$ used

7 preserved core plug samples to closely simulate the reservoir conditions while others $[3,4,6-8$,

$816,38,48,53,82]$ employed a sand-pack. Moreover, differences in the experimental procedures

9 may lead to different results in the same rock-fluid system.

\section{4.1. Type of the oil phase}

We first categorized the oil used into the two types as "Heavy oil" and "Light oil"

12 depending on viscosity. Since viscosity exerts a direct influence on flow, we consider it more

13 appropriate than density for oil classification. When the oil viscosity at ambient conditions is

14 higher than $100 \mathrm{cP}$, we consider it a heavy oil. Most researchers $[8,15,26,32,61,83]$ believe that

15 the flooding results for these two types are different; i.e., any judgment about the effect of

16 temperature without considering the oil type is not reasonable.

The temperature's impact on a heavy oil and light oil properties is significantly different.

18 Even a small increase in temperature can reduce the viscosity of heavy oil sufficiently $[2-7,17]$ to

19 affect the nature of flow. The temperature increase in thermal recovery operations causes the oil

20 viscosity to change by several orders of magnitude $[2-8,15,17,26,38]$. In waterflooding of water-

21 wet light oil systems, the mobility ratio is often favorable and the displacement is free of viscous

22 fingering [15, 26, 29, 32, 61]. However, viscous fingering is difficult to avoid in waterflooding of 
1 a heavy oil systems $[15,26]$. In light oil systems, the residual oil saturation can be reached typically

2 with five pore volumes of water injection while it can take hundreds of pore volumes in heavy oil

3 systems $[6,7,15,26]$. On the other hand, it is much easier to establish the irreducible water

4 saturation by oil flooding in heavy oil systems and the capillary end effects are small due to large

5 pressure drop $[6-8,15,26]$.

6 The literature review showed that majority of researchers used a light oil in their

7 investigations rather than a heavy crude oil. Approximately $40 \%$ of studies used a light refined oil

8 and 7\% used a light crude oil. Heavy refined oil and heavy crude oil were used in $11 \%$ and $29 \%$

9 of studies, respectively and $13 \%$ of studies did not specify the type of oil phase. Only a handful of

10 studies $[2,6,7,15,17,38,84,85]$ employed a heavy oil with viscosity higher than $1,000 \mathrm{cP}$ at

11 ambient temperature. The ambient temperature viscosity of oil used in the reviewed literature

12 varied from 0.64 to $1,000,000 \mathrm{cP}$. The values of density and viscosity of the oil phase, as reported

13 in the reviewed papers, are listed in Table 2.

Table 2. The oil phase properties employed in the literature.

\begin{tabular}{lcccc}
\hline Researcher & Year & Light/Heavy oil & $\begin{array}{c}\text { Viscosity } \\
(\mathbf{c P})\end{array}$ & $\begin{array}{c}\text { Density } \\
(\mathbf{g} / \mathbf{m L}) /(\mathbf{A P I})\end{array}$ \\
\hline Wilson [86] & 1956 & Light refined oil & N/A & N/A \\
\hline Odeh et al. [10] & 1959 & Light refined oil & $0.42-71.30\left(38^{\circ} \mathrm{C}\right)$ & $0.72-0.88\left(38^{\circ} \mathrm{C}\right)$ \\
\hline Edmondson [21] & 1965 & $\begin{array}{c}\text { Heavy crude/light } \\
\text { refined oil }\end{array}$ & $7.5-100\left(65.5^{\circ} \mathrm{C}\right)$ & $0.862-0.975\left(10^{\circ} \mathrm{C}\right)$ \\
\hline Davidson [82] & 1969 & Light refined oil & $65\left(37.7^{\circ} \mathrm{C}\right)$ & $0.863\left(23^{\circ} \mathrm{C}\right)$ \\
\hline Poston et al. [16] & 1970 & Light refined oil & $80.3-600\left(23.8^{\circ} \mathrm{C}\right)$ & $22.4-26\left({ }^{\circ} \mathrm{API}\right)$ \\
\hline Ehrlich [87] & 1970 & N/A & N/A & N/A \\
\hline Sinnokrot et al. [23] & 1971 & Light refined oil & $173\left(21.1^{\circ} \mathrm{C}\right)$ & $0.8645\left(21.1^{\circ} \mathrm{C}\right)$ \\
\hline Lefebvre du Prey [88] & 1973 & Light refined oil & $0.56-60\left(20^{\circ} \mathrm{C}\right)$ & $\mathrm{N} / \mathrm{A}$ \\
\hline
\end{tabular}




\begin{tabular}{|c|c|c|c|c|}
\hline Lo et al. [14] & 1973 & Light refined oil & $2-130\left(23^{\circ} \mathrm{C}\right)$ & N/A \\
\hline Weinbrandt et al. [20] & 1975 & Light refined oil & $65\left(37.8^{\circ} \mathrm{C}\right)$ & $0.863\left(23{ }^{\circ} \mathrm{C}\right)$ \\
\hline Abasov et al. [43] & 1976 & Crude oil & N/A & N/A \\
\hline Sufi et al. [51] & 1982 & Light refined oil & N/A & N/A \\
\hline Amaefule and Handy [89] & 1982 & Light refined oil & $0.6404-1.485\left(25^{\circ} \mathrm{C}\right)$ & $0.698-0.843\left(25^{\circ} \mathrm{C}\right)$ \\
\hline Torabzadeh et al. [24] & 1984 & Light refined oil & $0.9782\left(22^{\circ} \mathrm{C}\right)$ & $0.7531\left(22^{\circ} \mathrm{C}\right)$ \\
\hline Maini \& Batycky [15] & 1985 & Heavy crude oil & $16,400\left(22.8^{\circ} \mathrm{C}\right)$ & 0.9815 \\
\hline Miller \& Ramey [90] & 1985 & Light refined oil & $30\left(21^{\circ} \mathrm{C}\right)$ & N/A \\
\hline Kumar et al. [40] & 1985 & Light refined oil & $0.9782\left(22^{\circ} \mathrm{C}\right)$ & $0.7531\left(22^{\circ} \mathrm{C}\right)$ \\
\hline Bennion [44] & 1985 & Heavy refined/crude oil & N/A & N/A \\
\hline Polikar et al. [7] & 1986 & Heavy crude oil & $76.9 \pm 8.2\left(125^{\circ} \mathrm{C}\right)$ & $1.011-1.003\left(15^{\circ} \mathrm{C}\right)$ \\
\hline Nakomthap \& Evans [41] & 1986 & N/A & $30.91\left(21.1^{\circ} \mathrm{C}\right)$ & N/A \\
\hline Maini \& Okazawa [8] & 1987 & Heavy crude oil & $1,190\left(21^{\circ} \mathrm{C}\right)$ & N/A \\
\hline Closmann et al. [84] & 1988 & Heavy crude oil & $900-10,000\left(40^{\circ} \mathrm{C}\right)$ & $1.5-10.9\left({ }^{\circ} \mathrm{API}\right)$ \\
\hline Watson \& Ertekin [19] & 1988 & Light refined oil & N/A & N/A \\
\hline Maini \& Kokal [63] & 1989 & Refined oil & $500\left(20^{\circ} \mathrm{C}\right)$ & N/A \\
\hline Hawkins [91] & 1989 & Light refined oil & $0.725-2.0$ & $32\left({ }^{\circ} \mathrm{API}\right)$ \\
\hline Polikar et al. [6] & 1990 & Heavy crude oil & $\mathrm{N} / \mathrm{A}$ & N/A \\
\hline Frizzell [46] & 1990 & Heavy crude oil & N/A & $7-20\left({ }^{\circ} \mathrm{API}\right)$ \\
\hline Kumar \& Inouye [47] & 1994 & $\begin{array}{l}\text { Heavy/light } \\
\text { crude/refined oil }\end{array}$ & $\begin{array}{l}8.5-571.1\left(160^{\circ} \mathrm{C}\right) \\
34.4-11450\left(23^{\circ} \mathrm{C}\right)\end{array}$ & N/A \\
\hline Muqeem et al. [64] & 1995 & Heavy refined oil & $450\left(20^{\circ} \mathrm{C}\right)$ & $0.875\left(20^{\circ} \mathrm{C}\right)$ \\
\hline Akin et al. [37] & 1998 & Heavy refined oil & $220\left(21.1^{\circ} \mathrm{C}\right)$ & $0.878\left(21.1^{\circ} \mathrm{C}\right)$ \\
\hline Siddiqui et al. [92] & 1999 & Light refined oil & $1.86-68.27\left(37.8^{\circ} \mathrm{C}\right)$ & $0.761-0.865\left(37.8^{\circ} \mathrm{C}\right)$ \\
\hline Esfahani et al. [12] & 2004 & N/A & N/A & N/A \\
\hline Schembre et al. [50] & 2005 & Heavy crude/refined oil & $640\left(43^{\circ} \mathrm{C}\right)$ & N/A \\
\hline Bennion et al. [38] & 2006 & Heavy crude oil & $8,000-1,000,000$ & $7.5-12\left({ }^{\circ} \mathrm{API}\right)$ \\
\hline Wang et al. [11] & 2006 & Heavy/light crude oil & $430-13,550$ & N/A \\
\hline Sedaee Sola et al. [17] & 2007 & $\begin{array}{l}\text { Heavy/medium crude } \\
\text { oil }\end{array}$ & $4-16,000\left(37.8^{\circ} \mathrm{C}\right)$ & $12-26\left({ }^{\circ} \mathrm{API}\right)$ \\
\hline Hamouda et al. [13] & 2008 & $\mathrm{~N} / \mathrm{A}$ & N/A & N/A \\
\hline
\end{tabular}




\begin{tabular}{lcccc}
\hline Hamouda et al. [22] & 2008 & Light refined oil & N/A & N/A \\
\hline Ashrafi et al. [3] & 2012 & Heavy crude oil & $\begin{array}{c}200-900 \\
\left(50{ }^{\circ} \mathrm{C}\right)\end{array}$ & $\begin{array}{c}0.946-0.978 \\
\left(15{ }^{\circ} \mathrm{C}\right)\end{array}$ \\
\hline Akhlaghinia et al. [2] & 2013 & Heavy refined oil & $1,500\left(25^{\circ} \mathrm{C}\right)$ & $10.71\left({ }^{\circ} \mathrm{API}\right)$ \\
\hline Karaei et al. [93] & 2013 & Light refined oil & $800\left(25^{\circ} \mathrm{C}\right)$ & $\mathrm{N} / \mathrm{A}$ \\
\hline Ashrafi et al. [4] & 2014 & Heavy crude oil & $200-900\left(50^{\circ} \mathrm{C}\right)$ & $0.946-0.978\left(15^{\circ} \mathrm{C}\right)$ \\
\hline Vega et al. [18] & 2014 & Light crude oil & $7.20\left(45^{\circ} \mathrm{C}\right)$ & 0.87 \\
\hline Li et al. [94] & 2014 & Light crude oil & $0.8-2.9\left(23^{\circ} \mathrm{C}\right)$ & $\mathrm{N} / \mathrm{A}$ \\
\hline Nourmohhamad et al. [48] & 2015 & Light refined oil & $25.1\left(24^{\circ} \mathrm{C}\right)$ & $\mathrm{N} / \mathrm{A}$ \\
\hline Cao et al. [45] & 2016 & Light crude oil & $18.2\left(87^{\circ} \mathrm{C}\right)$ & $31.5\left({ }^{\circ} \mathrm{API}\right)$ \\
\hline Torabi et al. [42] & 2016 & Heavy/ light crude oil & $24.3,400.2\left(27^{\circ} \mathrm{C}\right)$ & $0.926-0.965$ \\
\hline Zeidani et al. [53] & 2016 & Heavy crude oil & N/A & N/A \\
\hline Mosavat et al. [5] & 2016 & N/A & N/A & N/A \\
\hline Zhang et al. [25] & 2017 & Light refined oil & $50\left(20^{\circ} \mathrm{C}\right)$ & N/A \\
\hline
\end{tabular}

\subsection{Type of rock}

Differences in the mineral content of rocks cause differences in rock surface properties,

3 such as surface charge and type of chemical components adsorbed on the rock surface. In the

4 presence of the oil and water phases in porous media, some components become adsorbed on the

5 pore surfaces. This can make the surface hydrophilic or hydrophobic, depending on what is

6 adsorbed. The contact angle, which is an indicator of wettability can be different for different types

7 of rocks using the same fluids. The fluid distribution within the pore space is affected by wettability

$8[29,36,56]$. Therefore, the rock type has significant effect on phase distribution and thereby on

9 the relative permeability of each phase $[26,27,29,35,36,56]$.

\section{$10 \quad$ 4.2.1. Sandstone vs. carbonate rock}

11 Most sandstone reservoirs are water-wet while the carbonates are mostly oil-wet or weakly

12 oil-wet. It is believed that the oil-wetness emerges after migration of oil into the reservoir by 
1 adsorption of polar components from the oil and this matter generally depends on the active

2 adsorption sites and polar/nonpolar components present in water and oil phases [41, 95]. However,

3 the adsorbed layer may become unstable and detach from the rock surface at high temperatures

$4 \quad[41,95]$, thereby causing the wettability to revert back to water-wet condition. Different types of

5 porous media, including sandstone, diatomite, carbonate, chalk and even Teflon, have been

6 employed to study the effect of temperature on relative permeability. Sand is the most often used

7 material either in the form of unconsolidated sand or consolidated sandstone cores. Amongst

8 carbonates, the dolomite and limestone are more prevalent than other types, like chalk. The polar

9 components adsorb more readily on the carbonate rock surfaces; therefore, continuing experiments

10 for a long time can alter the wettability of this type of rock. Besides wettability, other rock

11 properties, like compressibility and pore geometry, can also differ significantly in different rock 12 types.

13 The rocks used in relative permeability tests varied from thoroughly cleaned to fully

14 preserved or restored. It is apparent that the initial wettability varied. For instance, several

15 researchers cleaned the core by passing toluene through it followed by other solvents prior to each

16 experiment $[2,16,17,23,24,89]$. Furthermore, some core plugs were kept at a high temperature

17 in a furnace for 24 hours to remove all the organic materials and diminish the clay activity. In

18 contrast, the restored cores were used in several other investigations [12, 22, 47, 91, 92]. These

19 cores had been flooded with the oil phase to achieve the irreducible water saturation and kept at

20 the reservoir temperature for extended periods; hence, the polar components from oil could

21 become adsorbed on the surface and restore the wettability. Sometimes, the researchers employed

22 a preserved core or native one to eliminate any disturbances on the wettability as much as possible

$23[6,15,38,43,44,50,84]$. Approximately half of studies were carried out with clean porous media 
1 while preserved/restored/native cores were employed in $32 \%$ of them. Further details about the

2 type of porous media as well as their other properties are listed in Table 3.

3 Table 3. The properties of porous media used in different investigations of the effect of temperature on relative permeability.

\begin{tabular}{|c|c|c|c|c|}
\hline Researcher & Year & $\begin{array}{c}\text { Sand/carbonate } \\
\text { Clean/Preserved/Restored/Native }\end{array}$ & $\begin{array}{c}\text { Porosity } \\
\text { (\%) }\end{array}$ & $\begin{array}{c}\text { Permeability } \\
\text { (mD) }\end{array}$ \\
\hline Wilson [86] & 1956 & Clean core & 23.20 & 16.8 \\
\hline Odeh et al. [10] & 1959 & Consolidated core & $16.2-18.8$ & $2.1-405.0$ \\
\hline Edmondson [21] & 1965 & Clean consolidated Berea sandstone core & $20.5-21.1$ & $510-562$ \\
\hline Davidson [82] & 1969 & $\begin{array}{l}\text { Clean Tyler-grade Nevada sand and } \\
\text { aquarium gravel (47-60 mesh size) }\end{array}$ & 21.98 & 5,900 \\
\hline Poston et al. [16] & 1970 & $\begin{array}{l}\text { Clean unconsolidated Houston (170-200 } \\
\text { and 80-200 mesh size) }\end{array}$ & $34-40$ & 1,500 \\
\hline Ehrlich [87] & 1970 & N/A & $\mathrm{N} / \mathrm{A}$ & N/A \\
\hline Sinnokrot et al. [23] & 1971 & $\begin{array}{c}\text { Clean Berea and Bandera sandstone core } \\
\text { and clean carbonate core }\end{array}$ & $\begin{array}{c}19.7-21.5 \\
26.6\end{array}$ & $\begin{array}{c}152-610 \\
865\end{array}$ \\
\hline Lefebvre du Prey [88] & 1973 & Clear Teflon, Stainless steel and Alumina & $35-44.1$ & $60-6,000$ \\
\hline Lo et al. [14] & 1973 & $\begin{array}{c}\text { Clean consolidated Berea sandstone and } \\
\text { consolidated Teflon }\end{array}$ & $\begin{array}{c}21.6-24 \\
40.1-45.4\end{array}$ & $\begin{array}{c}560-620 \\
1,180-2,400\end{array}$ \\
\hline Weinbrandt et al. [20] & 1975 & Clean consolidated Boise sandstone core & $\begin{array}{l}18.75- \\
28.34\end{array}$ & $695-2,640$ \\
\hline Abasov et al. [43] & 1976 & Native consolidated media & N/A & N/A \\
\hline Sufi et al. [51] & 1982 & $\begin{array}{l}\text { Clean unconsolidated Ottawa sand } \\
\qquad(170-200 \text { mesh })\end{array}$ & $\mathrm{N} / \mathrm{A}$ & N/A \\
\hline $\begin{array}{c}\text { Amaefule and Handy } \\
{[89]}\end{array}$ & 1982 & Clean Berea sandstone core & $21.4-23.25$ & $239.2-472.1$ \\
\hline Torabzadeh et al. [24] & 1984 & Clear Berea sand stone core & $24.3-24.57$ & $643-969$ \\
\hline Maini \& Batycky [15] & 1985 & Native sandstone core plug & $31-35.3$ & $\mathrm{~N} / \mathrm{A}$ \\
\hline Miller \& Ramey [90] & 1985 & $\begin{array}{l}\text { 1) Clean unconsolidated Ottawa sand (100- } \\
200 \text { mesh size) and 2) consolidated Berea } \\
\text { sandstone core }\end{array}$ & $\begin{array}{l}\text { 1) } 38-38.3 \\
\text { 2) } 19\end{array}$ & $\begin{array}{l}\text { 1) } 6,750-7,160 \\
\text { 2) } 220\end{array}$ \\
\hline
\end{tabular}




\begin{tabular}{|c|c|c|c|c|}
\hline Kumar et al. [40] & 1985 & Clear Berea sandstone core & $24.3-24.57$ & $643-969$ \\
\hline Bennion et al. [44] & 1985 & Native Sparky sandstone core & 32 & 3,610 \\
\hline Polikar et al. [7] & 1986 & $\begin{array}{l}\text { Clean Ottawa sand } \\
\text { (200 mesh size })\end{array}$ & $32.1-35.8$ & $1,347-2,067$ \\
\hline Nakomthap \& Evans [41] & 1986 & N/A & 9.10 & N/A \\
\hline Maini \& Okazawa [8] & 1987 & $\begin{array}{l}\text { Clean Ottawa silica sand (140-170 mesh } \\
\text { size) }\end{array}$ & N/A & 3,000 \\
\hline Closmann et al. [84] & 1988 & Native Peace River sandstone core & $28.8-34.7$ & $39.9-653$ \\
\hline Watson \& Ertekin [19] & 1988 & Clean/restored Berea sandstone core & $20.6-24.5$ & $142.1-288.9$ \\
\hline Maini \& Kokal [63] & 1989 & Clean Ottawa sand (160-200 mesh size) & $\mathrm{N} / \mathrm{A}$ & 3,600 \\
\hline Hawkins [91] & 1989 & Restored granular carbonate core & $20-26$ & $9-26$ \\
\hline Polikar et al. [6] & 1990 & $\begin{array}{l}\text { Clean/native unconsolidated sand } \\
\qquad(170-230 \text { mesh size })\end{array}$ & $\begin{array}{l}34.0 \pm 1.0 \\
27.0 \pm 4.0\end{array}$ & $\begin{array}{l}958-2,070 \\
504-1,656\end{array}$ \\
\hline Frizzell [46] & 1990 & Clean unconsolidated sand & N/A & N/A \\
\hline Kumar \& Inouye [47] & 1994 & $\begin{array}{l}\text { Restored Berea sandstone and clean } \\
\text { unconsolidated media }\end{array}$ & 23 & $650-950$ \\
\hline Muqeem et al. [64] & 1995 & Clean Sand (140-200 mesh size) & N/A & 3,653 \\
\hline Akin et al. [37] & 1998 & $\begin{array}{l}\text { Clean unconsolidated South Belridge sand } \\
\qquad(100-200 \text { mesh size })\end{array}$ & 31 & 5,300 \\
\hline Siddiqui et al. [92] & 1999 & $\begin{array}{l}\text { Restored Berea and Brown consolidated } \\
\text { sandstone core }\end{array}$ & $18.1-21.6$ & $126-1,940$ \\
\hline Esfahani et al. [12] & 2004 & Restored Carbonate core plug & $8.20-24.90$ & $1.5-130$ \\
\hline Schembre et al. [50] & 2005 & Native Diatomite core plug & $55-67$ & $0.8-2.7$ \\
\hline Bennion et al. [38] & 2006 & Native unconsolidated McMurray sand & N/A & 4,567 \\
\hline Wang et al. [11] & 2006 & $\begin{array}{l}\text { Clean unconsolidated Ottawa sand } \\
\qquad(60-100 \text { mesh size })\end{array}$ & $35.6-36$ & $6,680-7,690$ \\
\hline Sedaee Sola et al. [17] & 2007 & Restored Carbonate core & $11-21$ & $0.2-3.0$ \\
\hline Hamouda et al. [13] & 2008 & N/A & 48.20 & 4.10 \\
\hline Hamouda et al. [22] & 2008 & Restored Chalk core & $43.3-50.8$ & $2.92-4.94$ \\
\hline Ashrafi et al. [3] & 2012 & $\begin{array}{l}\text { Clean unconsolidated glass beads } \\
\text { (1000 and } 300-425 \text { micron) }\end{array}$ & $28.8-34.26$ & $\begin{array}{c}90,000- \\
100,000 \\
40,000-45,000\end{array}$ \\
\hline
\end{tabular}




\begin{tabular}{|c|c|c|c|c|}
\hline Akhlaghinia et al. [2] & 2013 & Clean consolidated sandstone core & 19.27 & 1,560 \\
\hline Karaei et al. [93] & 2013 & Clean unconsolidated sand $(0.6-1.2 \mathrm{~mm})$ & $47-49.6$ & $\begin{array}{l}137,000- \\
474,000\end{array}$ \\
\hline Ashrafi et al. [4] & 2014 & $\begin{array}{l}\text { Clean unconsolidated Glass bead, sand and } \\
\text { Restored Bentheimer Sand stone core }\end{array}$ & $\begin{array}{l}20.88- \\
34.26\end{array}$ & $\begin{array}{c}40,000- \\
100,000 \\
13,800-15,800 \\
2,200-3,400\end{array}$ \\
\hline Vega et al. [18] & 2014 & Restored siliceous shale Diatomite core & 63 & 0.7 \\
\hline Li et al. [94] & 2014 & Restored limestone core & $\begin{array}{l}17.15- \\
17.49\end{array}$ & $0.314-0.358$ \\
\hline $\begin{array}{l}\text { Nourmohhamad et al. } \\
\qquad[48]\end{array}$ & 2015 & $\begin{array}{l}\text { Clean unconsolidated crushed carbonate } \\
\qquad(120-200 \text { mesh size })\end{array}$ & 39.5 & 5,210 \\
\hline Cao et al. [45] & 2016 & Restored sandstone core plug & $29.8-31.4$ & $305.3-966,2$ \\
\hline Torabi et al. [42] & 2016 & Clean Berea sandstone core plug & 25 & $2,730-2,890$ \\
\hline Zeidani et al. [53] & 2016 & Clean unconsolidated McMurray sand & N/A & N/A \\
\hline Mosavat et al. [5] & 2016 & N/A & $\mathrm{N} / \mathrm{A}$ & $\mathrm{N} / \mathrm{A}$ \\
\hline Zhang et al. [25] & 2017 & Sandstone core plug & $\begin{array}{c}16.15- \\
17.48\end{array}$ & $7.10-179.94$ \\
\hline
\end{tabular}

\subsection{Experimental procedures}

There are many differences in the experimental procedures used in different studies. These

3 procedures can be classified into two broad categories: steady-state method and displacement tests.

4 The steady-state measurements maintain isothermal conditions and involve continued injection of

5 a selected ratio of oil and water into the porous medium until the steady-state is reached in fluid

6 saturation, pressure drop, and produced fractional flow of the two fluids $[6,7,18,24,29,36,61$ -

7 63]. This procedure yields the relative permeability of oil and water at a specific water saturation.

8 The ratio of injected fluids is varied to obtain results at different saturations. The procedural

9 differences in studies relying on the steady-state method are in the technique used for determining 
1 the saturation under steady-state conditions and in the strategy used for minimizing the impact of

2 capillary end effects.

3 The displacement tests involve injection of one fluid to displace the other from the core,

4 generally starting from very high saturation of the displaced fluid [31, 36]. The test is continued

5 until the fractional flow of displaced fluid becomes practically zero [31, 36]. The relative

6 permeability is calculated from the recorded pressure drop and production history [31]. To study

7 the effect of temperature, most researchers have conducted such tests under isothermal conditions

8 and repeated them at other temperatures [2-4, 6-8, 12, 14, 15, 45, 48, 53]. However, some studies

9 have used incremental increase in temperature after reaching the residual oil temperature to

10 evaluate the effect of temperature $[17,23,37,90]$. It is an expeditious technique but limits the

11 reliability of results to evaluation of the residual oil saturation and the endpoint water permeability,

12 even though some authors have attempted to infer the whole relative permeability curve from such

13 tests $[17,37]$. The procedural differences in displacement tests are related to the methods used for

14 establishing the starting saturation, the displacement velocity used in the tests, techniques used in

15 monitoring the production and pressure drop, and whether or not the same core was used 16 repeatedly.

\section{4.4. Range of temperature used}

The effect of temperature was examined over varying temperature ranges in reviewed

19 studies; some involved a wide temperature range while others going up to only lukewarm

20 conditions. More than $55 \%$ of researchers conducted their experiments at temperatures below 120

$21{ }^{\circ} \mathrm{C}$ and only $16 \%$ of studies were performed at temperatures higher than $180{ }^{\circ} \mathrm{C}$. It is expected that

22 the changes that occur with increasing temperature would be amplified when the heating is

23 continued to higher temperatures. In other word, some phenomenon related to the high- 
1 temperature condition may not be observable unless the temperature changes adequately. To

2 clarify the distribution of temperature ranges in different studies, three different temperature ranges

3 were defined as "low temperature range" $\left(\mathrm{T}<90^{\circ} \mathrm{C}\right)$, "moderate temperature range" $\left(90^{\circ} \mathrm{C}<\mathrm{T}<180\right.$

$\left.4 \quad{ }^{\circ} \mathrm{C}\right)$ and "high temperature range" $\left(\mathrm{T}>180{ }^{\circ} \mathrm{C}\right)$ in this research. According to this analysis, only

$516 \%$ of studies were done in the high-temperature range, $37 \%$ in the moderate temperature range

6 and approximately half of studies were carried out in the low temperature range.

\section{5. EFFECT OF TEMPERATURE ON FLUID AND ROCK PROPERTIES}

As the system temperature is increased, the properties of both fluids and the rock can

9 change significantly. In this section, we examine the impact of temperature on properties that can

10 have substantial effect on measured relative permeability.

\section{$11 \quad$ 5.1. Interfacial tension (IFT)}

The IFT tends to decrease with increasing temperature [14-16] and this decrease can affect

13 the relative permeability. To isolate the impact of temperature induced IFT reduction on relative

14 permeability, it is necessary to find ways of changing only the IFT without changing the viscosity

15 ratio, which can be even more temperature sensitive. Amaefule and Handy [89] accomplished this

16 by evaluating the effect of IFT at constant temperature. Both steady-state and unsteady-state

17 relative permeability measurement were carried out at $25^{\circ} \mathrm{C}$. The drainage capillary pressure

18 measurements were also conducted using the centrifuge method. They used an aqueous phase

19 surfactant (Witco TRS $10-80$ and Klearfac AA-470) to decrease the IFT to $0.01 \mathrm{mN} / \mathrm{m}$ from the

20 value of $34.0 \mathrm{mN} / \mathrm{m}$ in the surfactant free system. A fired Berea sandstone core was used in these

21 tests. For steady-state relative permeability measurements, the gravimetric approach was used to

22 determine the saturations. They found that both the irreducible water saturation and the residual 
1 oil saturation decreased with the reduction of IFT. The residual oil saturation obtained by the

2 centrifuge capillary pressure test was different from the value observed in relative permeability

3 tests. This difference was attributed to differences in the fluid distribution. In the centrifuge test,

4 the bond number affected the fluid distribution rather than the capillary number. In steady-state

5 measurements, the relative permeability was considerably less affected by the IFT. The difference

6 between steady- and unsteady-state relative permeability was attributed to the differences in

7 distribution of fluid during the displacements.

$8 \quad$ Polikar et al. [7] reported that the interfacial tension decreased linearly with increasing 9 temperature. The IFT values measured in the study by Poston et al. [16] also reported a reduction

10 in IFT for three different oils at higher temperatures. Sinnokrot at al. [23] observed that the IFT

11 decreased from 53 dynes $/ \mathrm{cm}$ at ambient temperature to 32 at $163{ }^{\circ} \mathrm{C}$. A reduction of interfacial

12 tension with an increase in temperature was also mentioned by Zhang et al. [25]. In Hamouda et

13 al.[22] attributed lower residual saturation at higher temperature to higher capillary number due to

14 the decrease in the IFT. Torabzadeh et al. [24] reported that the decrease in IFT with increasing

15 temperature caused a reduction of irreducible water saturation. Edmondson [21] also accepted the

16 role of IFT change on modification of the relative permeability curves with increasing temperature.

17 In contrast to the above mentioned studies, Lo et al. [14] reported that the reduction of IFT

18 to less than 5 dyne/cm was not sufficient to change the oil and water relative permeability in their

19 study using tetradecane and water system. They found no temperature dependency of relative

20 permeability curves for tetradecane/water system at higher temperatures. The absence of

21 significant effect of decreased IFT in Lo et al. [14] study appears to be due to the use of steady-

22 state measurements in their work. As mentioned earlier, Amaefule \& Handy [89] also found that

23 decreased IFT affects the relative permeability measured by steady-state technique far less 
1 compared to the curves derived from displacement tests. In displacement tests, the impact of

2 reduced IFT occurs mostly by reduction of oil trapping, which requires only modest increase in

3 the capillary number.

\section{5.2. Viscosity Ratio}

5 The most prominent effect of increasing temperature in heavy oil systems is the dramatic

6 reduction of oil/water viscosity ratio, which may have substantial effect on the oil/water relative

7 permeability. Past researchers have investigated this issue two ways: (1) by using oils of different

8 viscosity at constant temperature and (2) by using the same oil at different temperatures. Odeh et

9 al. [10] conducted a theoretical analysis using a capillary tube bundle model of porous media by

10 postulating that the oil phase cannot displace the water phase completely; therefore, a thin water

11 film covers the rock surface. The thickness of this layer was found to be depended on the drag

12 force which was related to the oil viscosity and velocity distribution profile [10]. According to

13 their analysis, the effect of viscosity ratio decreases with increasing average pore radius and the

14 oil relative permeability becomes independent of viscosity ratio for average pore radius higher

15 than 4.5 microns.

Odeh et al. [10] also performed steady-state relative permeability measurements using four

17 different oils. The electrical resistivity of cores was used for saturation measurements. In

18 agreement with their theoretical model, the oil relative permeability (non-wetting phase) was a

19 function of saturation and viscosity ratio of two phases, while the wetting phase relative

20 permeability was only a function of its own saturation. Their experimental results showed that the

21 effect of viscosity ratio was more pronounced when the absolute permeability of the medium was

22 lower. They also reported a surprising finding that the effective permeability to a viscous oil could

23 be considerably larger than the absolute permeability measured with water. 
Lefebvre du Prey [88] postulated that the relative permeability to oil/water was related to

2 the magnitude of viscous and capillary forces; therefore, changing these factors caused a variation

3 in relative permeability. To prove this, they used three types of artificial porous media including

4 Teflon, Stainless steel, and Alumina. The contact angle measurements showed that Teflon was

5 strongly oil-wet, Alumina was strongly water-wet and stainless steel had intermediate wettability.

6 Displacement tests were carried out at $24^{\circ} \mathrm{C}$ using different oils. The IFT varied between $0.25-$

$7 \quad 350$ dynes/cm and the viscosity of different oils varied from 0.9 to $60 \mathrm{cP}$. They concluded that the

8 relative permeability to oil and water strongly depended on the balance between the capillary force

9 and viscous force, whereby the oil and water relative permeability sharply increased as the value

10 of $(\mu \nu / \sigma)^{-1}$ parameter decreases from $1,400,000$ to 5,100 . Their results showed that the residual

11 saturations of both the wetting phase and the non-wetting phase increased with increasing value of

$12(\mu \nu / \sigma)^{-1}$ parameter.

13 Wang et al. [11] investigated the effect of viscosity ratio on the two-phase oil/water relative

14 permeability using four stock tank oils and two crude oils diluted with kerosene at room

15 temperature. It was found that the higher viscosity oils gave larger residual oil saturations. In

16 addition, the relative permeability to oil and water as well as the endpoint relative permeability of

17 water were lower in experiments conducted with higher viscosity oils [11]. The residual oil

18 saturation increased linearly with $\log$ of viscosity and the irreducible water saturation decreased

19 as the viscosity of oil increased. The lubricating effect of water film at the irreducible water

20 saturation was suggested as a reason for the value of the endpoint oil relative permeability being

21 higher than 1 . The reduction of relative permeability to water and oil with increasing viscosity of

22 oil was attributed to more severe viscous fingering. 
Polikar et al. [6] stated that the shape of steady-state oil relative permeability in

2 unconsolidated sand is affected by the viscosity ratio. The impact of the oil/water viscosity ratio

3 on relative permeability measurements was also asserted by Ashrafi et al. [4], where the oil

4 viscosity reduction caused the residual oil saturation to decrease and the irreducible water

5 saturation to increase at higher temperatures. Esfahani et al. [12] also showed that the viscosity

6 ratio can alter the characteristics of relative permeability curves at elevated temperatures. Torabi

7 et al. [42] conducted two experiments with different oil viscosity while keeping the rest of

8 parameters constant to investigate the effect of oil viscosity on two-phase relative permeability

9 curves and, as can be seen in Figure 1, the oil viscosity had considerable impact on the relative

10 permeability. The relative permeability also depended on the injection flow rate in this study [42].

11 In yet another study, the decrease in the residual oil saturation at higher temperature was attributed

12 to the oil viscosity reduction of waxy oil, as reported by Cao et al. [45].

13 The involvement of viscosity ratio in determination of relative permeability was explained

14 by Sufi et al. [51], who generated same results by increasing the flow rate or decreasing the

15 viscosity at a given viscous force value. They showed that when any parameter affecting the

16 viscous force changes, the relative permeability might also change. Kumar \& Inouye [47]

17 emphasized that not only the viscosity ratio, but also the wettability condition must be considered

18 in examining the effect of temperature on relative permeability. Ashrafi et al. [3] suggested that

19 the change in irreducible water saturation could be due to some experimental errors; although they

20 believed that a large reduction of oil viscosity in comparison to water viscosity at higher

21 temperatures could also be a reason for such variation.

22 The effect of temperature on oil and water relative permeability was claimed to be

23 depended on the change in the viscosity ratio in a study conducted by Edmondson [21]. Lo et al. 
1 [14] also observed that the residual oil saturation decreased with temperature due to the reduction

2 of oil/water viscosity ratio at higher temperatures. The largest effect, in this study, was achieved

3 for the highest oil viscosity [14]. Li et al. [94] showed that higher oil/water viscosity ratio or higher

4 absolute permeability caused more oil to be trapped in porous media and resulted in higher residual

5 oil saturation. This result was attributed to more pronounced water fingering at higher oil/water

6 viscosity ratio and the difficulties in oil production after water breakthrough in higher permeability

7 cases [94].

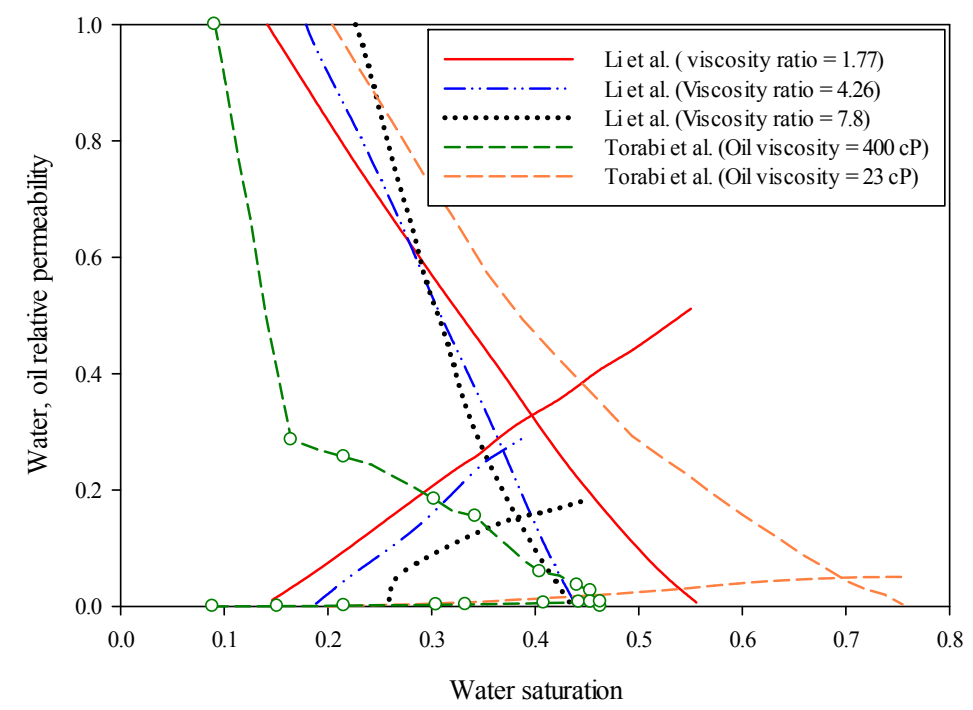

Figure 1. The effect of oil viscosity or viscosity ratio on two phase relative permeability [42, 94].

The opinion of a vast majority of researchers on the effect of viscosity ratio appears to be

11 that it has a profound effect on oil-water relative permeability inferred from displacement tests.

12 Yet there is no theoretically sound reason why this should be the case. If the fundamental

13 assumption of the primacy of surface forces in controlling the fluid distribution within the pore

14 space (which is the only justification for considering the relative permeability to be dependent only

15 on saturation) is still valid, then the relative permeability should not change with oil viscosity. It

16 suggests that there is a breakdown of the assumption of capillarity controlled fluid distribution in 
1 displacement tests involving high viscosity of oil. The fact that many of aforementioned studies

2 have mentioned viscous fingering to be at least partly responsible for the observed effects of

3 viscosity ratio confirms that the surface forces were not controlling the fluid distribution. It also

4 means that the relative permeability curves inferred from displacements involving viscous

5 fingering would be applicable only to displacements that have similar extent of fingering. It then

6 becomes apparent that the steady-state method, which is generally not affected by viscous

7 fingering, would be more reliable for measuring relative permeability in systems involving high

$8 \mathrm{oil} /$ water viscosity ratio $[26,31,61,96]$.

The literature review also shows that the high oil viscosity reduces the irreducible water

10 saturation and increases the residual oil saturation. These observations appear to be related to

11 experimental conditions imposed in such tests. The reported residual oil saturation in most studies

12 is the practical residual saturation that occurs when the fractional flow of oil becomes lower than

13 a threshold value. When the oil viscosity is very high, the practical residual saturation may be

14 much higher than the true residual saturation. On the other side, the irreducible water saturation is

15 lower with high viscosity oil primarily due to much higher pressure gradients used during the

16 injection of viscous oil, which not only provides more efficient displacement but also suppresses

17 the capillary end effects at the production end.

\subsection{Wettability and Contact Angle}

The preference of the rock surface for oil or water as the wetting fluid stems from difference

20 in the surface energies of rock-water and rock-oil interfaces. When the surface energy of rock-

21 water interface is much smaller than that of rock-oil interface, the rock is strongly water-wet and

22 vice-versa [35]. The equilibrium distribution of fluids in the pore space under capillarity-controlled

23 conditions would be the configuration that minimizes the total surface free energy of rock-fluid 
1 and fluid-fluid interfaces. Therefore the wettability of rock surface plays an important role in

2 controlling the fluid distribution and thereby determining the two-phase relative permeability [26, $329,32,35,56]$. The angle of contact of the oil-water interface at the line of contact with the solid surface

5 is determined by the force balance involving solid-water, solid-oil and oil-water interfacial 6 tensions [35, 97]. The interfacial tension of the solid and non-wetting phase interface is balanced

7 by the sum of the interfacial tension of the solid and wetting phase interface plus the projection of 8 the fluid-fluid interfacial tension on the solid surface [35, 98]. A small contact angle means a larger 9 projection of the fluid-fluid interfacial tension is needed to counterbalance the solid and non10 wetting phase interfacial tension with the two opposing forces [99]. Thus, the oil-water contact 11 angle provides a measure of the difference between the surface energies of rock-oil and rock-water 12 interfaces relative to the value of the oil-water interfacial tension and it can be used to assess the 13 wettability of solid surfaces. However, it should be kept in mind that when the oil-water interfacial 14 tension is very small, the difference between the rock-oil and rock-water interfacial energies would 15 be very small, irrespective of the value of contact angle (assuming a stable contact angle exists). 16 In this situation, the surface should be considered neutrally wet regardless of the value of contact 17 angle and the contact angle is no longer a good indicator of surface wettability. Figure 2 shows the 18 governing forces on a water drop immersed in oil. 


$$
\sigma_{\mathrm{wo}} \cdot \cos (\theta)+\sigma_{\mathrm{sw}}=\sigma_{\mathrm{so}}
$$

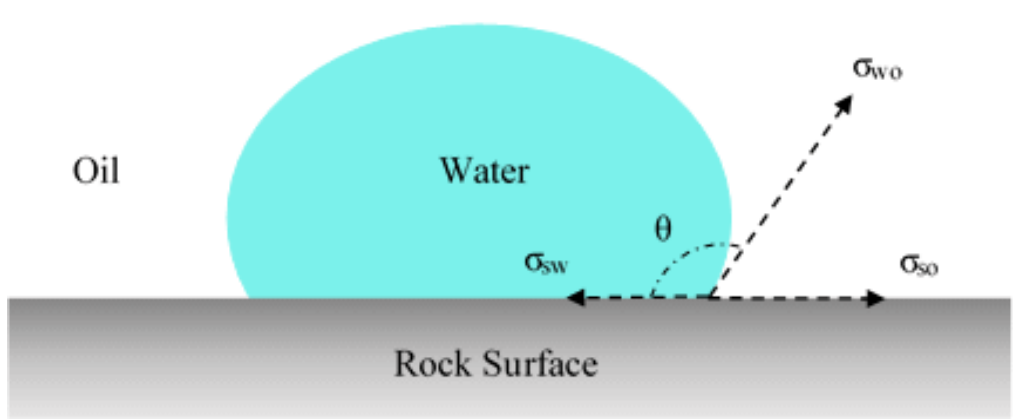

Figure 2. Schematic of a water drop showing the quantities in the Young equation [98].

4 on wettability $[14,16]$. Other methods for characterizing the wettability of porous media include

5 the Amott method [100] and the USBM method [101]. The wettability of a porous medium can

6 also be assessed by examining the oil-water relative permeability curves and applying the Craig's

7 rules [83]. In this method, the intersection point of oil and water relative permeability curves as

8 well as the endpoint relative permeability to oil and water are indicators of the wettability [83].

9 Like the oil-water interfacial tension, the surface energies of rock-oil and rock-water

10 interfaces can also change with temperature. Hence, the rock wettability can change with

11 increasing temperature. Numerous investigators [4, 12-20] have endeavored to detect whether or

12 not any wettability alteration occurred during their relative permeability measurements at elevated

13 temperatures. Poston et al. [16] used contact angle measurements to evaluate the wettability

14 alteration with temperature. Based on the results presented in Figure 3 for three different oil phases,

15 the glass surface showed a tendency toward more water-wetness with an increase in temperature.

16 Lo et al. [14] also measured contact angles at different temperatures and found no variation for

17 Teflon, but a decreasing trend from $40^{\circ}$ to $28^{\circ}$ for the quartz surface. 


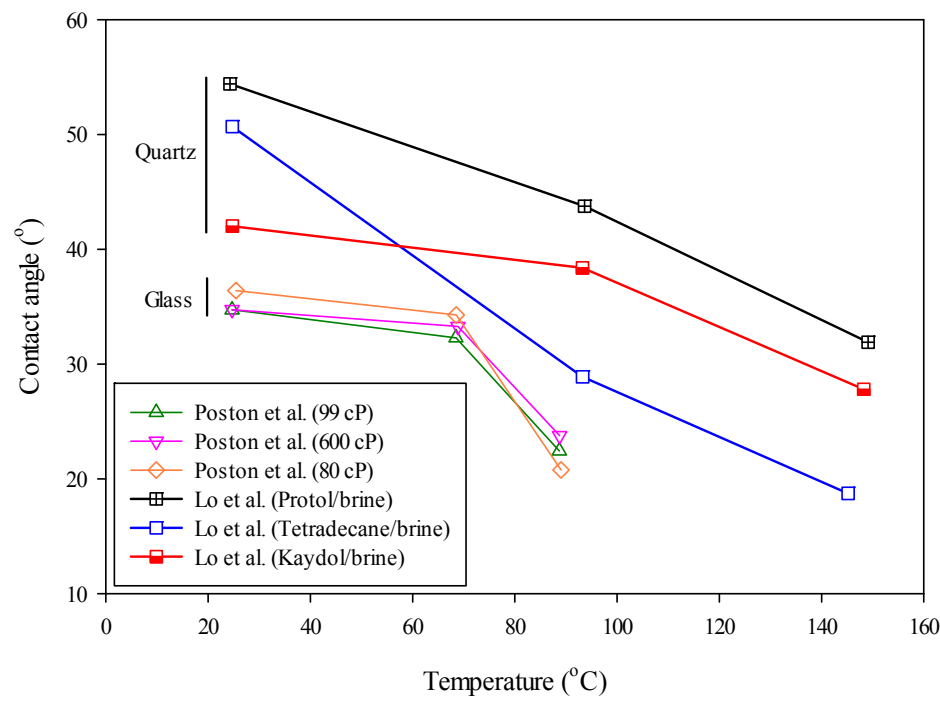

Figure 3. The variation of contact angle for some systems reported in the literature $[14,16]$.

4 and USBM [101] tests. The Amott-Harvey index for core samples at ambient temperature was in

5 the range of -0.479 and -0.287 , which indicated an intermediate to weak oil-wet system, while an

6 oil-wet characteristic was observed at the higher temperature of $103{ }^{\circ} \mathrm{C}$, with the Amott-Harvey

7 index between -0.669 and -0.478 . Table 4 summarizes the results of our literature survey regarding

8 reported wettability alterations with increasing temperatures for various systems in different

9 studies. Further information about the type of porous media and fluid properties in these systems

10 are listed in Table 2 and Table 3.

Table 4. The variation of rock surface wettability at higher temperatures.

\begin{tabular}{lccccc}
\hline Researcher & Year & $\begin{array}{c}\text { Trend of } \\
\text { wettability }\end{array}$ & Researcher & Year & $\begin{array}{c}\text { Trend of } \\
\text { wettability }\end{array}$ \\
\hline Poston et al. [16] & 1970 & More water wet & Sinnokrot et al. [23] & 1971 & $\begin{array}{c}\text { More water wet } \\
\text { (Sandstone) }\end{array}$ \\
\hline Lo et al. [14] & 1973 & No change & Weinbrandt et al. [20] & 1975 & More water wet \\
\hline Sufi et al. [51] & 1982 & No change & Torabzadeh et al. [24] & 1984 & More water wet \\
\hline
\end{tabular}




\begin{tabular}{|c|c|c|c|c|c|}
\hline Maini \& Batycky [15] & 1985 & More oil wet & Miller \& Ramey [90] & 1985 & No change \\
\hline Kumar et al. [40] & 1985 & More water wet & Nakomthap \& Evans [41] & 1986 & $\begin{array}{l}\text { More water wet } \\
\text { (Based on the } \\
\text { model) }\end{array}$ \\
\hline Maini \& Okazawa [8] & 1987 & More oil wet & Closmann et al. [84] & 1988 & More oil wet \\
\hline Watson \& Ertekin [19] & 1988 & More oil wet & Hawkins [91] & 1989 & No change \\
\hline Akin et al. [37] & 1998 & No change & Esfahani et al. [12] & 2004 & More oil wet \\
\hline Schembre et al. [50] & 2005 & More water wet & Bennion et al. [38] & 2006 & More water wet \\
\hline Sedaee Sola et al. [17] & 2007 & $\begin{array}{l}\text { More oil wet } \\
\text { (Heavy } \\
\text { oil/Limestone), } \\
\text { More water wet } \\
\text { (Light } \\
\text { oil/Dolomite) }\end{array}$ & Hamouda et al. [22] & 2008 & $\begin{array}{l}\text { More water wet at } \\
\text { the temperature } \\
\text { lower than } 80^{\circ} \mathrm{C} \\
\text { and more oil wet at } \\
\text { the temperature } \\
\text { above } 80^{\circ} \mathrm{C}\end{array}$ \\
\hline Karaei et al. [93] & 2013 & No change & Vega et al. [18] & 2014 & More water wet \\
\hline Nourmohhamad et al. [48] & 2015 & No change & Zhang et al. [25] & 2017 & More water wet \\
\hline
\end{tabular}

\section{THE EFFECT OF TEMPERATURE ON RELATIVE PERMEABILITY CURVES}

As discussed above, several rock-fluid properties that can affect the relative permeability

3 might change with increasing temperature. These include the surface energies of rock-fluid and

4 fluid-fluid interfaces (which determine the wettability and affect the dominance of surface forces

5 in controlling fluid distribution) and the viscosity of each fluid. The effect of temperature on

6 relative permeability will therefore depend on whether or not changes in these properties in a

7 specific system are substantial enough to affect the fluid distribution. Obviously, not all systems

8 would be expected to display a change in relative permeability with an increase in temperature.

9 Furthermore, a distinction should be made between cases where the change is sufficient to affect

10 the fluid distribution but the dominance of surface forces is not compromised and cases in which

11 the dominance of surface forces no longer exists. In the latter case, one has to question whether

12 the concept of relative permeability is still appropriate to model the two-phase flow. Besides these 
1 issues, the variations in techniques used and the simplifying assumption made in interpreting the

2 experimental results can also affect the outcomes.

3 Looking back critically into the previous investigations conducted over past six decades it

4 becomes apparent that this issue is still not resolved and we need more research into the effect of

5 temperature on reservoir systems. Although a large number of experimental studies have been

6 carried out in different ranges of temperature and pressure using different porous media, the

7 findings often contradict each other. Some researchers $[6,7,37,48,51,86,90,91]$ have concluded

8 that the relative permeability to oil and water is independent of temperature even when the

9 temperature increases by more than $200^{\circ} \mathrm{C}$, while others $[2,20,42]$ reported that even a small

10 increase in temperature can change the oil/water relative permeability curves significantly. One

11 can easily conclude that the effect of temperature would be system dependent but it is not clear in

12 what type of reservoir systems one should expect the relative permeability to change more with

13 temperature and in which direction.

14 To gain an insight into the reported effects of temperature on oil-water relative

15 permeability, we have used six characteristics of relative permeability curves: (1) irreducible water

16 saturation, (2) residual oil saturation, (3) endpoint relative permeability to water, (4) endpoint

17 relative permeability to oil, (5) shape of water relative permeability curve, and (6) shape of oil

18 relative permeability curve. The following subsections review the reported results on how

19 temperature affects these parameters in different types of systems.

\section{6.1. Irreducible water saturation}

21 The irreducible water saturation $\left(\mathrm{S}_{\mathrm{wi}}\right)$ is the lowest water saturation achieved by displacing

22 water from the porous medium by injecting oil. At this saturation, water is no longer mobile, hence 
1 its saturation cannot be decreased further by displacement by oil. Actually, at least in strongly

2 water-wet systems, the water remains continuous in the form of a wetting film on pore surfaces

3 and its mobility does not become zero at a well-defined saturation $[29,56]$. Therefore, $\mathrm{S}_{\mathrm{wi}}$

4 measured in such displacement tests, in reality, is the saturation at which the water mobility

5 becomes negligible compared to the oil mobility under the employed tests conditions. The

6 measured $S_{w i}$ can vary significantly with the capillary number used in the displacement (or the

7 employed pressure gradient, which is an important factor in the capillary number) $[24,88,89$,

8 102]. Therefore, it is important to keep in mind that when similar flow rates are used, the pressure

9 gradient becomes much smaller at higher temperatures due to much lower oil viscosity and some

10 of the observed changes in $S_{w i}$ with temperature might be related to changes in the pressure

11 gradient. The irreducible water saturation is one of the factors used in determining the wettability

12 state of the rock surface from relative permeability curves according to Craig's rules [83]. It should

13 be noted that unless the test conditions were similar in terms of the capillary number (or at least

14 the pressure gradient) used in the oil flood to establish $\mathrm{S}_{\mathrm{wi}}$, the change in $\mathrm{S}_{\mathrm{wi}}$ is not necessarily

15 related to a change in wettability.

The change of $\mathrm{S}_{\mathrm{wi}}$ with temperature has been extensively investigated in a large number of

17 experimental studies $[3,4,12,14-20,23-25,38,40-42,44-46,51,53,94]$. Although the results of

18 different studies sometimes contain conflicting results regarding the effect of temperature, there is

19 considerable consistency in the trend of $\mathrm{S}_{\mathrm{wi}}$ with temperature. In the majority of reported studies

$20[3,4,14-20,23-25,38,40-42,44-46,51,94], S_{w i}$ increases with temperature. However, several

21 other experimental studies $[6-8,17,23,24,40,47,48,86,90,91]$ have found no variation with

22 temperature and a decrease in the irreducible water saturation with increasing temperature has also

23 been mentioned in some studies $[12,53]$. 
Figure 4 compares some of the reported data from several different studies $[3,4,14,20$,

$225,42,51]$ on the effect of temperature on irreducible water saturation. $S_{\text {wi }}$ was measured after 15

$3 \mathrm{PV}$ of oil injection at a rate of $0.1 \mathrm{~mL} / \mathrm{min}$ by Zhang et al. [25]. To reduce the impact of capillary

4 end effect, a filter paper was used at the production end of the core. In this research [25], the

5 increase of temperature from $25{ }^{\circ} \mathrm{C}$ to $100{ }^{\circ} \mathrm{C}$ caused dramatic increase in $\mathrm{S}_{\text {wi. }}$ Since same flow

6 rate was used at all temperatures but the viscosity of oil decreased by almost two orders of

7 magnitude, the pressure gradient would be much lower in the high temperature tests and at least a

8 part of this increase in irreducible water saturation is due to the reduced pressure gradient.

Torabi et al. [42] found that as the temperature increased by $18^{\circ} \mathrm{C}, \mathrm{S}_{\mathrm{wi}}$ increased from 0.19

10 to 0.29 . Their heavy oil viscosity dropped by almost one order of magnitude with this increase in

11 temperature. Li et al. [94] also found that the value of $S_{w i}$ in low permeability limestone increased

12 from 0.2 to 0.3 with temperature increase of only $14{ }^{\circ} \mathrm{C}$. Lo et al. [14] found that $\mathrm{S}_{\text {wi }}$ increased

13 with temperature more dramatically when a viscous mineral oil was used compared to

14 measurements with tetradecane. It is worth noting that the viscosity decrease was more pronounced

15 for mineral oil than for tetradecane [14].

The displacement tests performed by Ashrafi et al. [4] demonstrated that $\mathrm{S}_{\mathrm{wi}}$ increased as

17 the temperature increased. This increase of the $\mathrm{S}_{\mathrm{wi}}$ with temperature was attributed to water/oil

18 viscosity ratio increase. They also found that a similar increase in $\mathrm{S}_{\mathrm{wi}}$ could be observed when the

19 oil viscosity is decreased at constant temperature, which confirms the role of the capillary number.

20 Weinbrandt et al. [20] also reported a significant increase in $\mathrm{S}_{\mathrm{wi}}$, with increasing temperature. Sufi

21 et al. [51] also observed an increase in the irreducible water saturation with temperature. They

22 found that similar increase in $S_{w i}$ could be obtained by decreasing the flow rate or decreasing the

23 oil viscosity in the test without changing the temperature. By plotting $S_{w i}$ against viscous force 
1 (product of flow rate and oil viscosity), they were able to show that the variation of $\mathrm{S}_{\mathrm{wi}}$ resulting

2 from changes in flow velocity, fluid change and temperature change fitted the same curve. They

3 attributed the change in $S_{w i}$ to change in the capillary end effect at the production end and more

4 efficient displacement at higher viscous force [51].

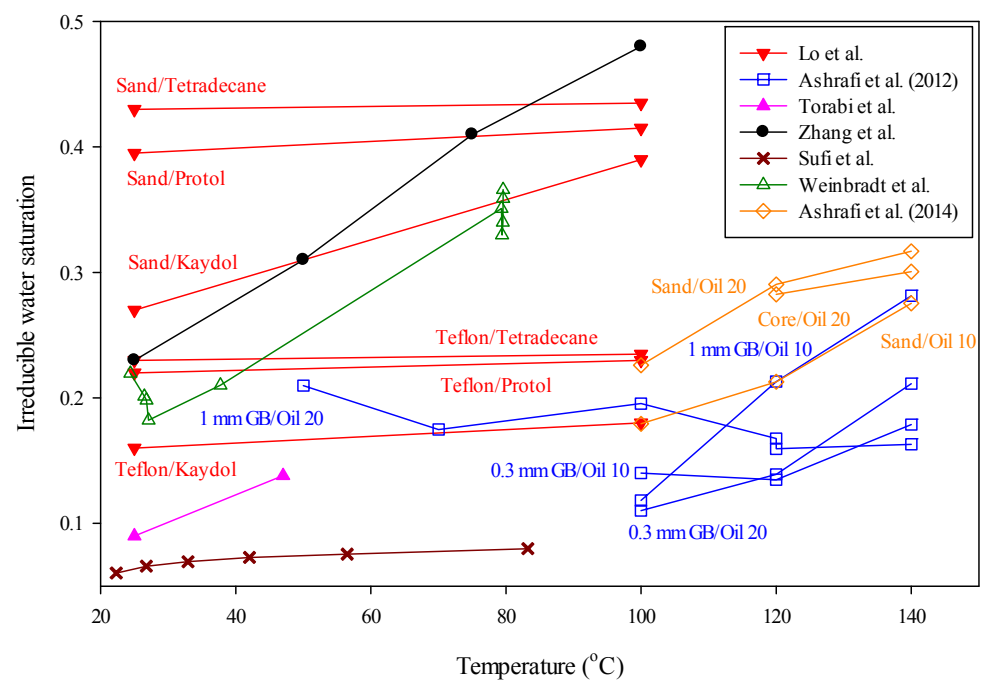

Figure 4. The increasing trend of irreducible water saturation with temperature for different systems [3, 4 ,

Amaefule and Handy [89] established the irreducible water saturation by pushing $40 \mathrm{PV}$

9 of oil into the core plug and found that $S_{w i}$ increased with decreasing capillary number. Poston et

10 al. [16] found that the increase in $S_{\mathrm{wi}}$ is less pronounced for clean unconsolidated sand compared

11 to a reservoir sand. In addition, they found that the effect of temperature on irreducible water

12 saturation was only partially reversible [16]. After exposure to high temperature, the repeat

13 measurement in the same medium displayed slightly higher $\mathrm{S}_{\mathrm{wi}}$. They suggested that a part of the

14 increase occurs due to increase in water wettability, which is not reversible and the rest is due to

15 the change in water/oil viscosity ratio. Note that to calculate the irreducible water saturation, water

16 volume was recorded until the water cut reached $1 \%$ or less. It was mentioned that the measured

$17 \mathrm{~S}_{\mathrm{wi}}$ is not actually equal to the real irreducible water saturation but the same trend with temperature 
1 would be expected [16]. They suggested that there is significant change in the capillary pressure

2 curve with increasing temperature due to changes in the interfacial tension and wettability, and

3 this may be a contributing factor in the observed change in $\mathrm{S}_{\text {wi }}$.

The CT scan analysis done by Vega et al. [18] showed that the average water saturation at

5 zero water fractional flow increased from 0.16 to 0.62 as the temperatures increased from $45^{\circ} \mathrm{C}$ to

$6230{ }^{\circ} \mathrm{C}$. The tendency of the system toward more water-wetness by increasing the temperature was

7 suggested to be responsible for it. Temperature dependency of two phase oil/water relative

8 permeability and capillary pressure was investigated by Sinnokrot et al. [23] using restored-state

9 approach applied to drainage and imbibition capillary pressure measurement at temperatures

10 between room temperature and $163{ }^{\circ} \mathrm{C}$. They employed the capillary pressure measurement to

11 determine whether the hypothesis proposed by Poston et al. [16] for capillary pressure

12 modification at higher temperature is true or not. The temperature was increased incrementally in

13 order to prevent any disruption of the system. Moreover, the core plugs were fired to oxidize

14 organic material within the core. The Brook and Corey [103] relative permeability model was used

15 for drainage relative permeability curve estimation. The results of this study [23], shown in Figure

165 , confirmed that the irreducible water saturation increases linearly with temperature for sandstone

17 core, although at temperatures higher than $121.11^{\circ} \mathrm{C}\left(250^{\circ} \mathrm{F}\right), \mathrm{S}_{\mathrm{wi}}$ data deviated from the linear

18 behavior. Moreover, the increasing behavior of $\mathrm{S}_{\mathrm{wi}}$ intensified at $148.89{ }^{\circ} \mathrm{C}\left(300{ }^{\circ} \mathrm{F}\right)$, which

19 suggests that the wettability of sand stone can be changed toward more water wetness, especially

20 at temperatures higher than $148.89^{\circ} \mathrm{C}\left(300^{\circ} \mathrm{F}\right)$. Interestingly, Swi did not change with temperature

21 for the limestone core plug. 


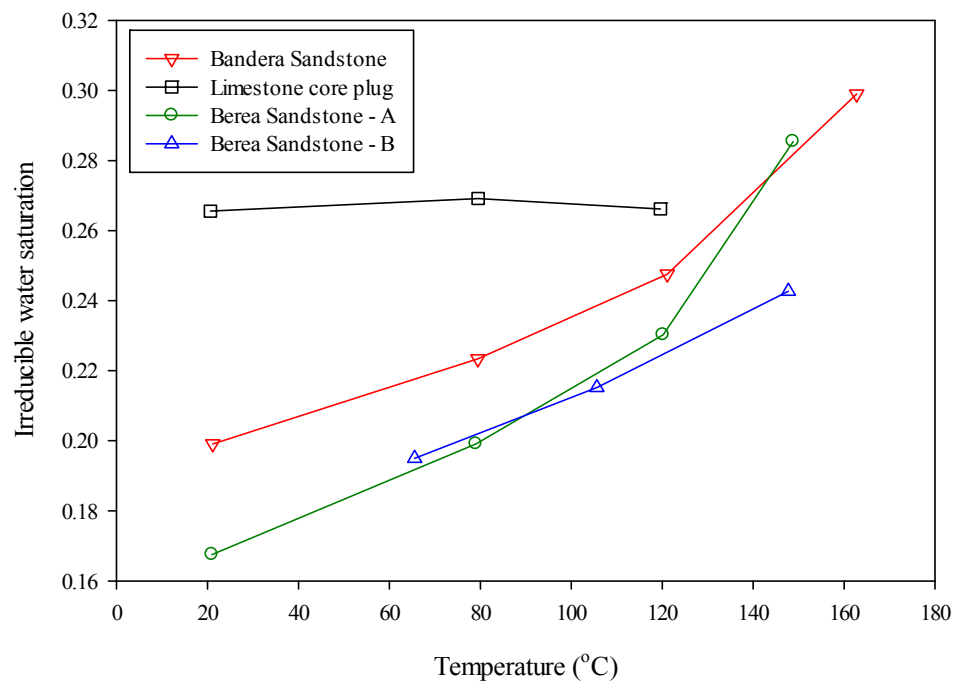

Figure 5. Different trend of $S_{w i}$ with temperature obtained by Sinnokrot et al. [23].

Torabzadeh et al. [24] reported the results of irreducible water saturation at temperatures

4 up to $175{ }^{\circ} \mathrm{C}$ for the system containing $2 \% \mathrm{NaCl}$ brine and $\mathrm{n}$-dodecane in fired Berea sandstone

5 core plugs. They found that $50 \mathrm{PV}$ of oil injection was required to achieve the irreducible water

6 saturation within high IFT system. An increase in water wetness in high IFT system was suggested

7 as a possible reason for the observed increase of irreducible water saturation with temperature. The

8 irreducible water saturation data from this study are shown in Figure 6. The low IFT system

9 displayed only minor changes in the irreducible water saturation with temperature [24]. It is

10 apparent that the capillary numbers were much higher in low IFT displacements and this lead to

11 low irreducible water saturation at all temperatures.

An extensive analysis on 43 data sets of two phase relative permeability, measured over 15

13 years at high and low temperatures under hot water or steam injection, was performed by Bennion

14 et al. [38]. The unsteady-state method was used in 41 out of 43 sets and steady-state approach was

15 used for the other two. The temperature varied from 10 to $280^{\circ} \mathrm{C}$. It is notable that all experiments

16 were performed with low enough flow rates to minimize fines migration. Their results, shown in 
1 Figure 7, indicate that the irreducible water saturation increases linearly with temperature, as

2 represented by Equation 2. They also suggested that the initial water saturation at low temperature

3 might be a function of absolute permeability.

$$
S_{w i}=8.3 \times 10^{-4} T\left({ }^{\circ} \mathrm{C}\right)+0.135533
$$

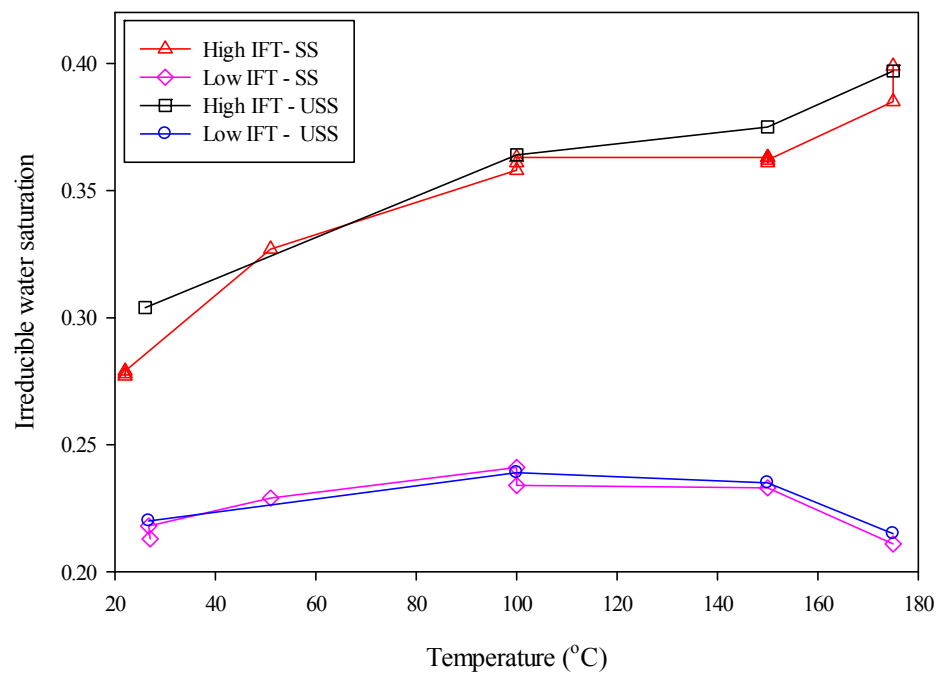

$5 \quad$ Figure 6. The irreducible water saturation versus temperature obtained by Torabzadeh et al. [24].

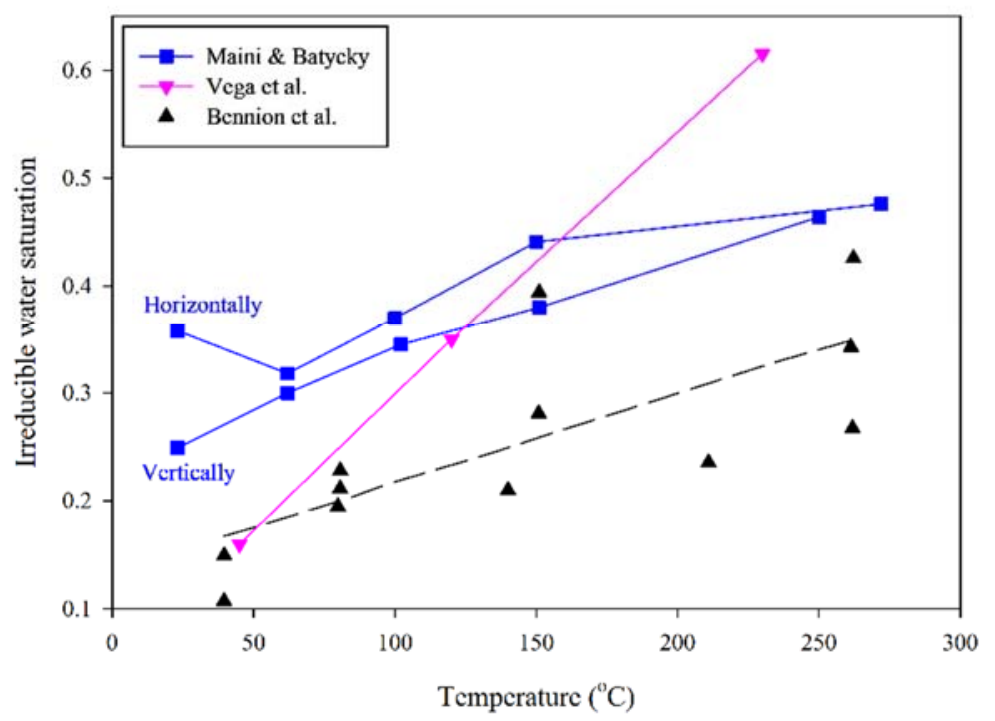

$7 \quad$ Figure 7. The irreducible water saturation behavior at different temperatures reported by different 8 researchers $[18,38,90]$. 
The effect of temperature on two phase oil/water relative permeability was also examined

2 by Sola et al. [17] using unsteady-state technique. The temperature influence was investigated over

3 a wide range of $37.7-260^{\circ} \mathrm{C}$ under high pressure between $4.8-13.8 \mathrm{MPa}$. They employed two

4 different procedures to evaluate the effect of temperature on relative permeability, where the

5 temperature increased incrementally after reaching a specific criterion in some tests and remained

6 constant during the whole tests in others. To determine the relative permeability curve, a black oil

7 simulator with power law Corey model [104] was employed for history matching. The irreducible

8 water saturation increased linearly with temperature in heavy crude oil systems but was

9 independent of temperature for a lighter oil of $10 \mathrm{cP}$ viscosity. Figure 8 presents their $\mathrm{S}_{\mathrm{wi}}$

10 measurements in three different systems.

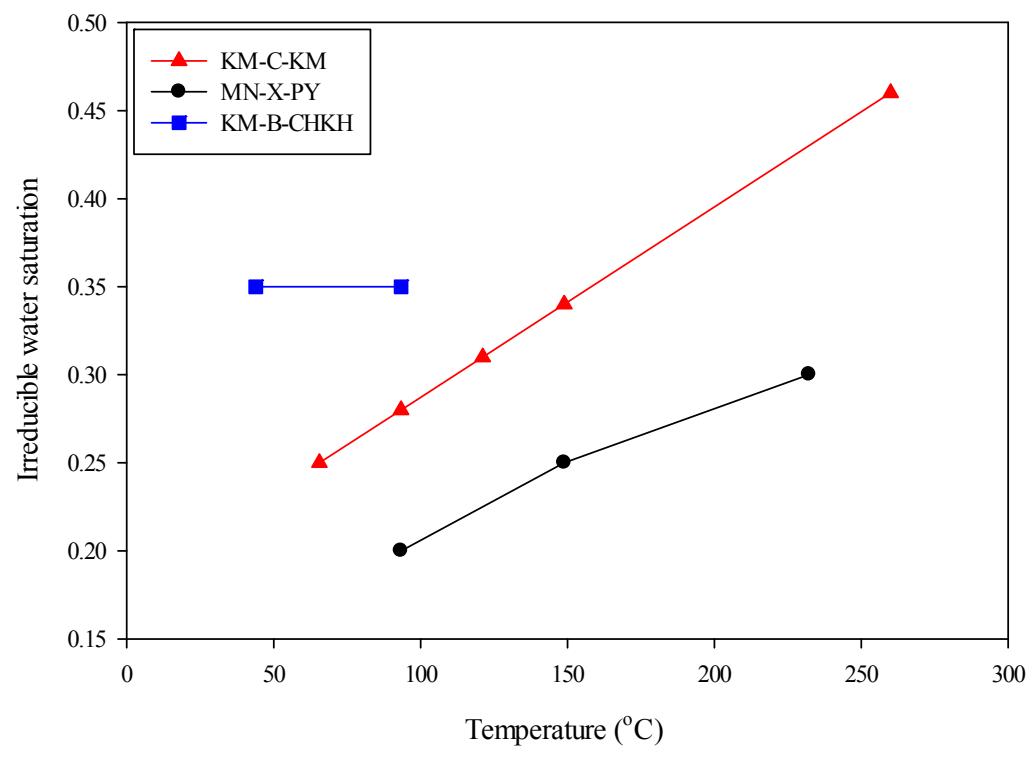

12 Figure 8. The effect of temperature on irreducible water saturation reported by Sola et al. [17].

13 The following empirical correlation for estimating the irreducible water saturation versus

14 temperature was proposed by Frizzell [46] for unconsolidated sand. 


$$
S_{w i}(\%)=0.8472 \times\left({ }^{\circ} A P I\right)+0.0925 \times\left({ }^{\circ} F\right)-0.00062 \times k_{w}+2.77
$$

Kumar et al. [40] proposed two separate empirical correlations for estimating the

2 irreducible water saturation as function of temperature for normal IFT and low IFT systems, based

3 on the data published by Torabzadeh et al. [24].

4 For high IFT:

$$
S_{w i}=0.1828 \times T^{0.1427}
$$

5 For low IFT:

$$
S_{w i}=0.1828 \times T^{0.1427}+0.49835 \sigma-0.17196
$$

Ashrafi et al. [3] studied the effect of temperature on heavy oil-water relative permeability

7 in the temperature range of 50 to $140{ }^{\circ} \mathrm{C}$ using two different blends of Athabasca bitumen and $\mathrm{n}$ -

8 dodecane. The Sandra simulator using five different relative permeability models (Burdine [75],

9 Corey [104], Sigmund \& McCaffery [105], Chierici [106] and LET correlation [107]) was used

10 for history matching of unsteady-state relative permeability measurements. The results generally

11 showed that the irreducible water saturation increased with temperature [3]. It was pointed out that

12 the effect of flow rate on displacement performance was examined by increasing the injection flow

13 rate and making sure that the ultimate oil recovery did not change with flow rate [3].

14 An earlier study performed by Wilson [86] in 1956 showed that the irreducible water 15 saturation was independent of temperature. The temperature was increased from 32 to $71{ }^{\circ} \mathrm{C}$ while 16 the pressure was varied from 0.2 to $34.46 \mathrm{MPa}$ to capture the pressure effect. Their findings are 17 not too surprising, since the change in water/oil viscosity ratio, with kerosene as the oil and such 18 small increase in temperature, would not be that large. 
Another major attempt to examine the issue was made by Polikar et al. [6]. The effect of

2 temperature on relative permeability was examined using both the steady-state and unsteady-state

3 techniques. Their results indicated that the irreducible water saturation was not significantly

4 affected by temperature. Although there were some variations in their experiments, they believed

5 them to be attributable to porous media heterogeneities. In another study, Polikar et al. [7] reported

6 similar results for irreducible water saturation variation with temperature. The reduction of $S_{w i}$ at

7 higher temperatures observed by Polikar et al. [7] was attributed to solubilization of water phase

8 into the large volume of the oil phase that passed through the porous medium. The values of

9 irreducible water saturation in Polikar et al. $[6,7]$ studies are shown in Figure 9.

10 Hawkins [91] injected $10 \mathrm{PV}$ of Blandol to obtain irreducible water saturation at ambient

11 condition before heating the core to $100{ }^{\circ} \mathrm{C}$ for 1000 hours to restore the wettability of the core

12 and prepare it for an experiment at reservoir conditions. They reported that the irreducible water

13 saturation was independent of temperature. This is not surprising since the water was displaced by

14 injecting a viscous refined oil at ambient conditions in all tests.

15 Nourmohammad et al. [48] suggested that 15 PVs of oil injection is adequate to achieve

16 the irreducible water saturation, which was reported to be 0.15 in all tests. It is notable that the

17 temperature range in this study only varied from room temperature to $85{ }^{\circ} \mathrm{C}$. The change in

18 oil/water viscosity ratio was relatively small over the range of temperature used and apparently

19 not enough to show any clear-cut effect of temperature on $\mathrm{S}_{\mathrm{wi}}$.

In the study of Maini \& Okazawa [8], the oil phase was first injected into the sand pack at

21 room temperature and then core was heated to the desired temperature, during which only oil was

22 produced from the core. Therefore, the water saturation increased only due to the thermal

23 expansion of immobile water, which was not a large change due to the low irreducible water 
1 saturation (5\%) at room temperature. In Miller \& Ramey [90], the irreducible water saturation at

2 room temperature was determined by 2 PV of oil injection into $100 \%$ water saturated core.

3 However, for higher temperature runs, the drainage displacement of water was conducted starting

4 from residual oil saturation. They reported no change in irreducible water saturation by increasing

5 temperature up to $150{ }^{\circ} \mathrm{C}$ in the unconsolidated sand pack and consolidated Berea sandstone core.

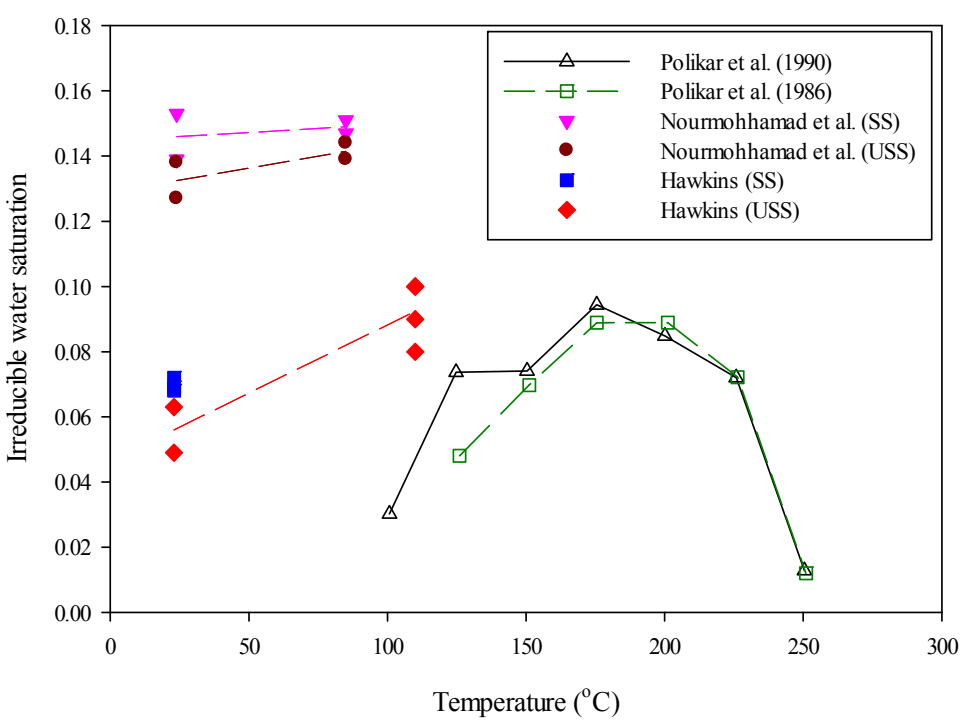

7 Figure 9. Comparison of the irreducible water saturation variation with increasing temperature $[6,7,48$, 91].

9 Reduction of irreducible water saturation with increasing temperature has been also 10 reported in some studies. Two interesting papers about the reduction of irreducible water saturation 11 with increasing temperature are the studies by Esfahani et al. [12] and Zeidani et al. [53] in 2004 12 and 2016; respectively. Esfahani et al. [12] applied the graphical JR [108] method to translate the 13 transient production data acquired from unsteady state displacements. Five to ten pore volumes of 14 oil were injected into the core to estimate the saturation endpoint. They reported a decrease in 15 irreducible water saturation at reservoir condition and attributed it to increase in oil wetness at 16 elevated temperature. Zeidani et al. [53] conducted SAGD experiments in a linear sand-pack at 
1 three different temperatures of $180^{\circ} \mathrm{C}, 200^{\circ} \mathrm{C}$, and $220^{\circ} \mathrm{C}$. The production and pressure response

2 data were then converted to relative permeability curve using the history matching approach. The

3 Corey [104] relative permeability model was used in CMG simulator to match the production data;

4 however, the obtained simulation result did not fit the experimental result that well. The irreducible

5 water saturation was used as an adjustable parameter in history matching of different tests [53].

6 The measured irreducible water saturation for these two studies is displayed in Figure 10.

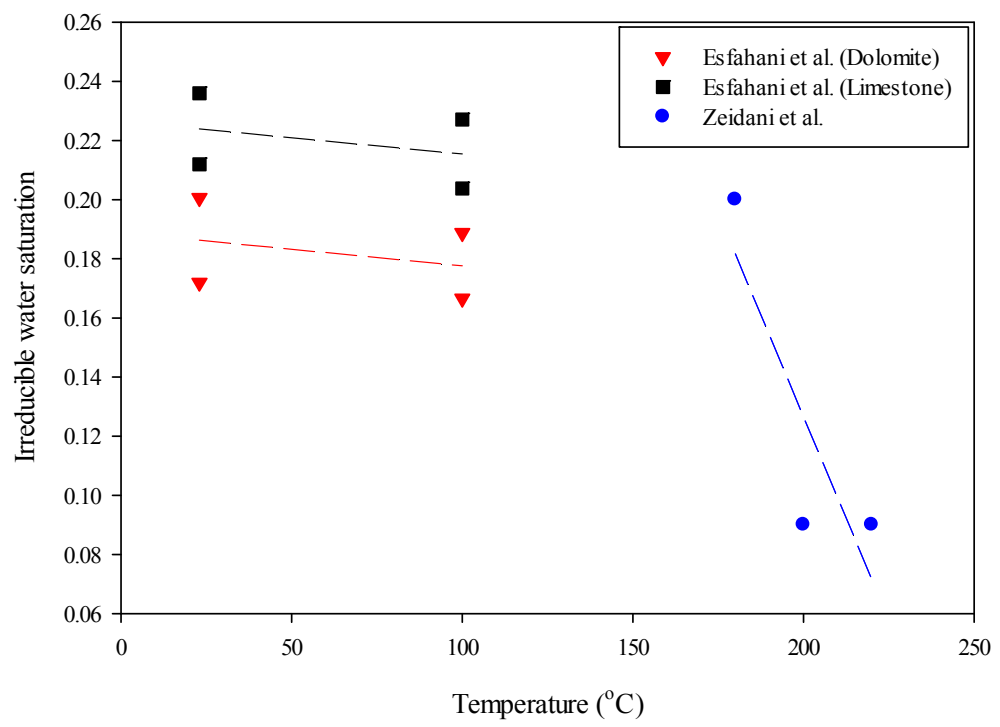

8 Figure 10. The decreasing trend of irreducible water saturation with temperature [12, 53].

9 The results obtained by Hamouda et al. [22] are also interesting. They conducted unsteady-

10 state relative permeability tests. In order to alter the rock wettability, they used either n-decane

11 with fatty acid additives [0.005 M SA (Steric acid) and 0.005M PODA (18-phenyloctadecanoic

12 acid)] or n-decane without any additives. The wettability of cores were established by aging them

13 at a temperature of $90{ }^{\circ} \mathrm{C}$ before starting each experiment. The temperature range during the

14 experimental measurements using distilled water with $0.005 \mathrm{M} \mathrm{SA}$ was from 23 to $130^{\circ} \mathrm{C}$. The

15 results, presented in Figure 11, show that the irreducible water saturation first increased slightly at

16 the temperature of $50{ }^{\circ} \mathrm{C}$ and then decreased more significantly up to the temperature of $130{ }^{\circ} \mathrm{C}$. 
1 This matter has also been investigated by Gonari and Hamouda [109], Karoussi and Hamouda

2 [110]; however, they paid more attention to other factors such as interaction potential in

3 calcite/distilled water that becomes more repulsive at temperature over $70{ }^{\circ} \mathrm{C}$. It explains how, in

4 such systems, the wettability can change non-monotonically with increasing temperature.

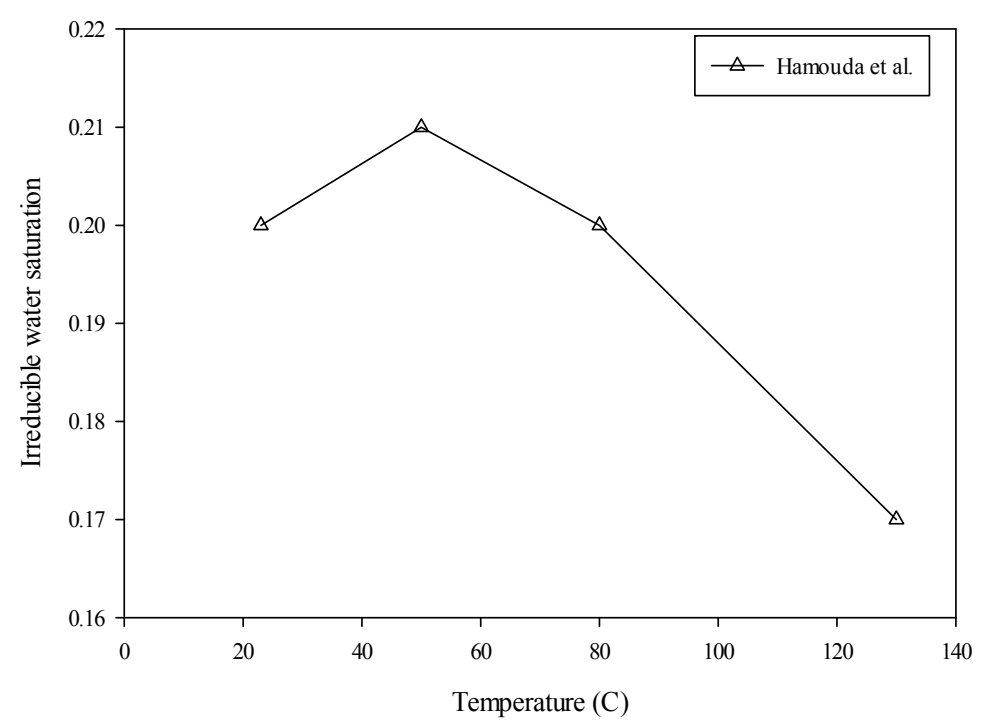

5

11 oil of $45^{\circ} \mathrm{C}$ pour point in reservoir core plugs. Their results, in Figure 12, show a steep increase in

$12 \mathrm{~S}_{\mathrm{wi}}$ with increasing temperature. Results of Poston et al. [16], which were discussed earlier are also

13 included in this figure for comparison and show a more modest increase with temperature. 


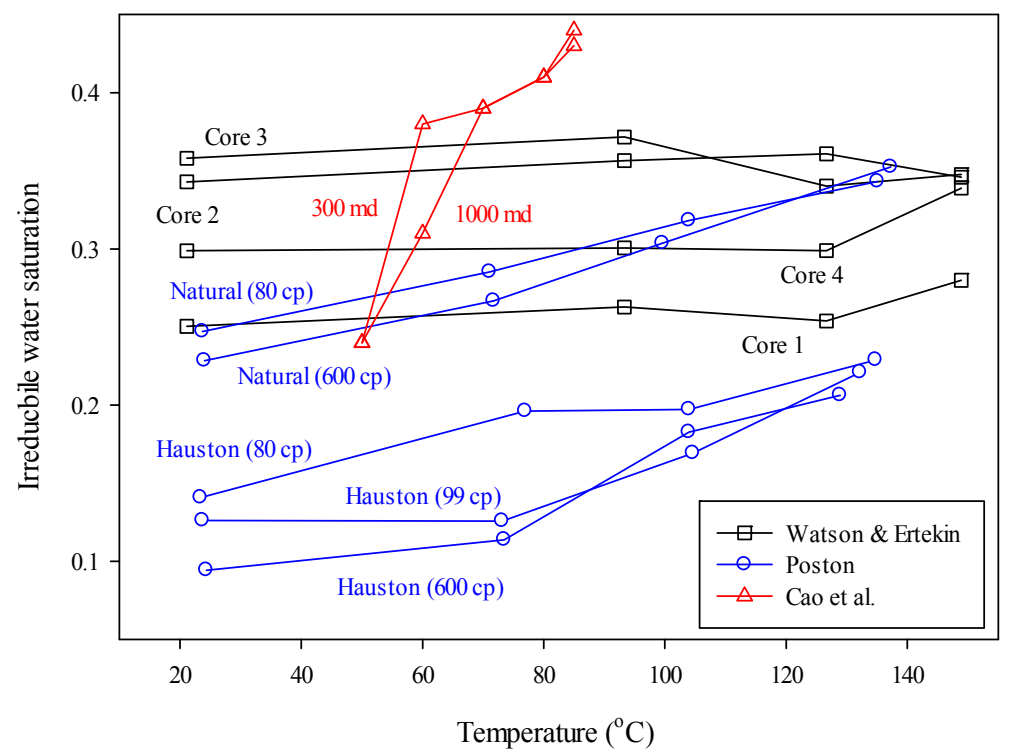

Figure 12. The variation of irreducible water saturation with temperature $[16,19,45]$.

The preceding review of reported results tends confirms what was said in the beginning of

4 this section that, in water-wet systems, the water relative permeability does not become zero at a

5 well-defined saturation and the experimentally determined $S_{\text {wi }}$ represents the water saturation at

6 which the mobility of water becomes "negligible" compared to the mobility of oil under two-phase

7 flow conditions. The actual definition of "negligible" is not same in all studies but in most studies

8 it represents the condition at which the water mobility is of the order of $0.1 \%$ to $1 \%$ of the total

9 mobility. Kumar \& Inouye [47] stated that during the unsteady-state relative permeability

10 measurements, the irreducible water saturation can be achieved when the fractional flow of oil at

11 the outlet of the core reaches $99.9 \%$. They pointed out that the true irreducible water saturation

12 due to the presence of thin film flow cannot be obtained at all; therefore, the value of irreducible

13 water saturation depends on total injected volume. The produced brine to oil volume ratio of 400

14 was accepted by Watson \& Ertekin [19] as a critical value for determining the value of irreducible

15 water saturation. Many researchers have used a minimum number of pore volumes of oil injected 
1 as a criterion for getting to the irreducible water saturation. For example, Akhlaghinia et al. [2]

2 continued the oil injection to around $15 \mathrm{PV}$ to achieve the irreducible water saturation. Cao et al.

3 [45] obtained the irreducible water saturation by displacing water with $10 \mathrm{PV}$ of oil. Weinbrandt

4 et al. [20] also injected $10 \mathrm{PV}$ of oil into the water saturated core to obtain the practical irreducible 5 water saturation.

6 Another important issue in experimental measurements of $\mathrm{S}_{\mathrm{wi}}$ is the necessity of 7 minimizing capillary end effect at the production end. Water saturation remains high near the 8 production end due to the need for capillary pressure to be equal on both sides of the production 9 face of the rock under two phase flow. The capillary pressure is practically zero outside the rock, 10 so it needs to be equally small at the face, inside the rock also. The zone of high water saturation 11 extends some distance into the medium and it is imperative to make this zone very small compared

12 to the length of the sample in measuring $S_{w i}$, so that the impact of such high water saturation at the 13 end becomes negligible. This requires using high pressure gradient during oil injection to compress

14 the capillary end effect zone. In our own work, we have found the pressure gradient of $50 \mathrm{psi} / \mathrm{ft}$ to 15 be adequate and safe to use in most systems [26]. Higher pressure gradients sometimes lead to 16 fines migration damage [38]. Unfortunately, many of the reviewed studies did not pay enough 17 attention to this capillary end effect problem, especially in high temperature tests. Theoretically, the irreducible water saturation depends on three factors: 1) the rock surface

19 wettability, 2) pore geometry and 3) the capillary number used in the displacement of water by oil

20 injection. In studies using clean unconsolidated sand and refined oil, it is unlikely that the

21 wettability and pore geometry will change with increasing temperature. The capillary number used

22 is an operational parameter that appears to be responsible for the increase in $S_{w i}$ with temperature

23 seen in many studies with such clean systems. In natural reservoir systems, the wettability can 
1 change with temperature due to change in the concentration of adsorbed species on pore surfaces

$2[29,38,95]$. In some systems, the pore geometry may also change due to rock-fluid interactions

3 and thermal stress effects. In such systems, the value of $S_{w i}$ can change significantly with

4 temperature. However, many of the reported studies with such systems have paid insufficient

5 attention to the impact of capillary number, which can change the apparent irreducible saturation

6 even when there is no change in wettability.

\section{6.2. Residual oil saturation}

Among many studies of the effect of temperature on two-phase flow that have been

9 reported during the past sixty years, a large majority of them $[3,4,14,16-18,20,21,23-25,38$,

$1040,42,44-46,50,53,82,94]$ report that the residual oil saturation decreases as temperature is

11 elevated. Nonetheless, several studies $[8,17,19,37,47,48,51,86,90,91]$ suggest that the residual

12 oil saturation was independent of temperature. Very often, the measurement of this parameter is

13 not exact due to experimental limitations in the measurements of relative permeability, such as

14 limitations in the number of pore volumes that can be injected. The following review provides

15 some insight into the current understanding about the effect of temperature on Sor.

An early study was conducted by Edmondson [21] in 1965 to assess the behavior of Sor

17 and water/oil relative permeability ratio at high temperatures. Five different oil types including

18 three mineral oils (No.5 white oil, No.10 white oil, and No.1 white oil) and two crude oils were

19 employed to measure the two-phase relative permeability at temperatures ranging from 24 to 260

$20{ }^{\circ} \mathrm{C}$ in two consolidated cores. Prior to the experiment, the cores were heated up to $400{ }^{\circ} \mathrm{C}$ for 24

21 hours to completely remove any organic material and desensitize the clays, and make the cores

22 water wet. The definition of the residual oil saturation was based on the slope of the $\mathrm{k}_{w} / \mathrm{k}_{\mathrm{o}}$ value

23 versus water saturation as the point where the value $\mathrm{k}_{w} / \mathrm{k}_{\mathrm{o}}$ approaches infinity [21]. Between 8 to 
$110 \mathrm{PV}$ of hot water was injected in the displacements, which caused the fractional flow of water

2 to oil to be 100:1 for the crude oil systems and 1000:1 for the white oil systems. The residual oil

3 saturation was found to decrease with increasing temperature.

Davidson [82] conducted eleven unsteady-state relative permeability measurements. The

5 temperature range was 22 to $282{ }^{\circ} \mathrm{C}$. The residual oil saturation decreased with temperature

6 irrespective of the absence or presence of the initial water saturation. Davidson [82] also

7 investigated the effect of flow rate and initial water saturation and found that the flow rate did not

8 affect Sor. His observations are shown in Figure 13. Two values are shown for each of the two

9 systems (with and without initial water saturation), one obtained without any correction and the

10 other corrected for solubility of water in the oil. Poston et al. [16] also investigated the

11 temperature's impact on relative permeability to oil and water at temperatures up to $135^{\circ} \mathrm{C}$. The

12 residual oil saturation was assumed to occur when the effluent had less than 1 volume $\%$ of the oil

13 phase and it showed a decreasing trend with increasing temperature. This study suggested that a

14 permanent wettability alteration had occurred at high temperature, whereas the previous

15 researchers had considered the wettability alteration to be reversible [16]. Moreover, the higher

16 viscosity oil showed greater temperature dependency of Sor. Their results of the residual oil

17 saturation are shown in Figure 14.

18 Torabzadeh et al. [24] also reported a reduction of Sor at higher temperatures using eight

19 unsteady-state and twenty two steady-state relative permeability measurements in two systems,

20 one exhibiting high interfacial tension and the other with low IFT. As shown in Figure 14, the

21 reduction of Sor was smaller in the high IFT system and more pronounced in the low IFT system.

22 It was mentioned that due to the low oil viscosity, fluid flow was stable and a piston-like

23 displacement occurred [24]. 


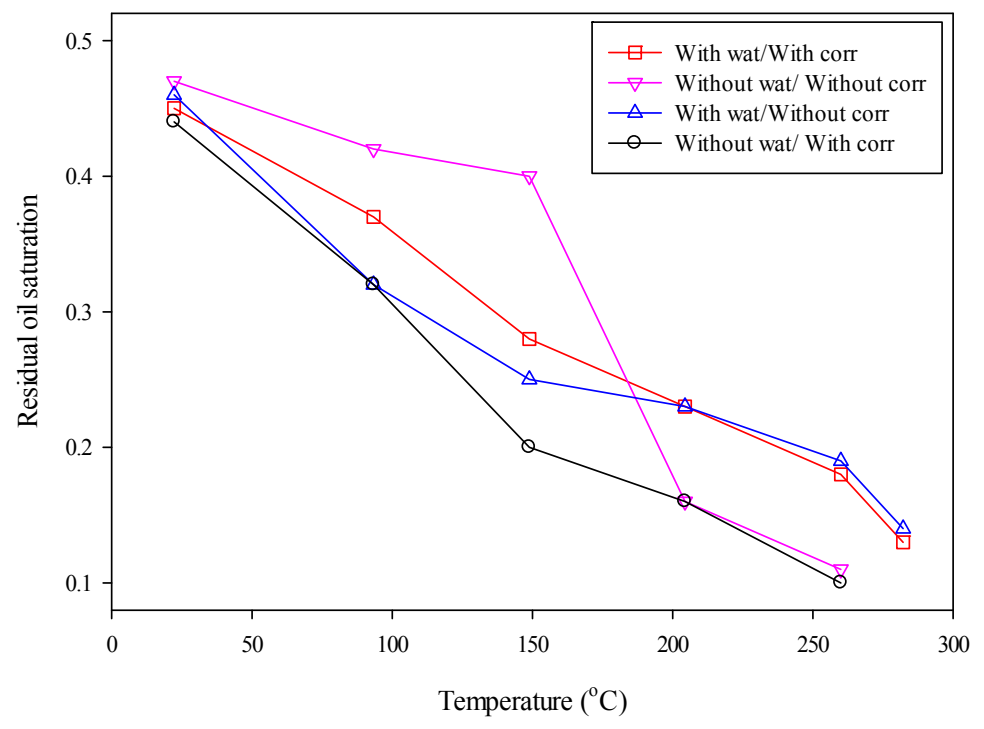

Figure 13. The residual oil saturation versus temperature obtained by Davidson [82].

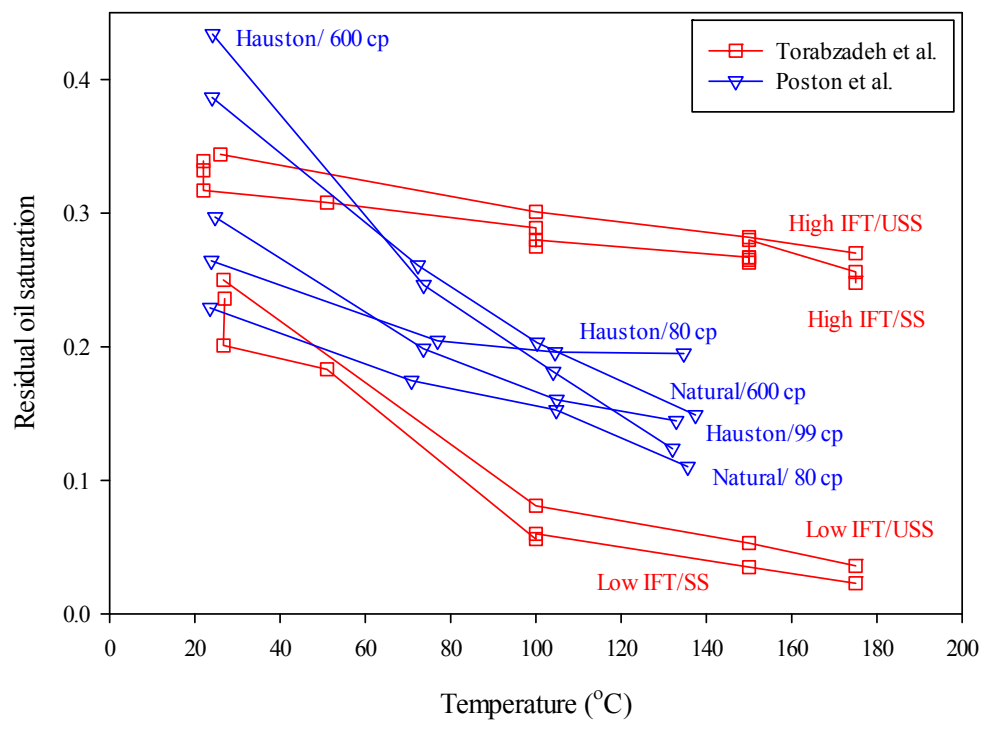

Figure 14. The reduction of residual oil saturation with temperature increasing $[16,24]$.

Lo et al. [14] used Teflon core as a strongly oil-wet medium and Berea sandstone core as

6 a water-wet material to conduct steady-state relative permeability measurements. They installed a

7 porous plate at the end of the core to minimize the impact of capillary end effects. An important

8 issue in the steady-state measurement is accurate measurement of the saturation in the core, which

9 they handled with the electrical resistivity measurements. Furthermore, they checked the material 
1 balance using the volume of collected effluent and compared it with the electrical resistivity

2 measurement. They employed tetradecane and two white oils as the oil phase and $3 \% \mathrm{NaCl}$ for the

3 water phase. Prior to each test, the Berea core was heated up to $871{ }^{\circ} \mathrm{C}$ to eliminate the organic

4 material and stabilize clays. They reported that Sor decreased with temperature and a larger

5 decrease was seen for the higher oil viscosity. Their results are presented in Figure 15.

6 Many other researchers have evaluated the effect of temperature on residual oil saturation

7 and found similar results. For example, Li et al. [94] reported that the residual oil saturation and

8 the irreducible water saturation are a function of temperature when the temperature increase is high

9 enough. In this study, the temperature range used was $86-100{ }^{\circ} \mathrm{C}$ at $30 \mathrm{MPa}$ pore pressure. The

10 cores were aged with simulated oil for three days at each experimental condition. The results were

11 consistent with the previous researchers $[3,4,14,16-18,20,21,23,24,38,40,42,44-46,50,82]$

12 and the residual oil saturation decreased as temperature increased. Li et al. [94] attributed this

13 result to the wettability alteration causing increased water-wetness of the rock at higher

14 temperatures and they supported this idea on basis of the results from past studies $[8,111]$. They

15 suggested that higher oil/water viscosity ratio causes more oil to be trapped in porous media and

16 results in higher residual oil saturation due to more pronounced water fingering [14]. Figure 16

17 includes the residual oil saturation results from this study.

Cao et al. [45] reported that the value of the residual oil saturation decreased from 0.569 to

190.236 when the temperatures was increased from $50{ }^{\circ} \mathrm{C}$ to $85^{\circ} \mathrm{C}$. They mentioned the possibility

20 of higher flow resistivity due to the wax deposition at temperatures below $70{ }^{\circ} \mathrm{C}$ [45]. The results

21 were similar in two sandstone cores of widely different permeability, as illustrated in Figure 16.

22 Ashrafi et al. [4] also reported that $\mathrm{S}_{\text {or }}$ tended to decrease at higher temperatures. Sinnokrot et al.

23 [23] observed the same trend in the Berea sandstone system, as shown in Figure 17. 


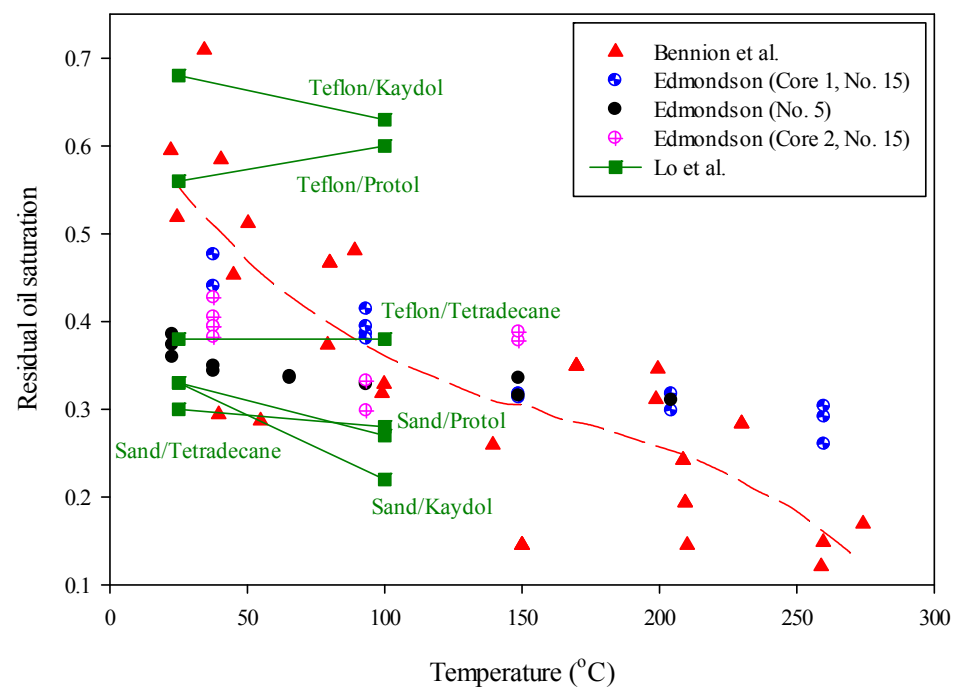

Figure 15. Comparison of the residual oil saturation trends at higher temperatures [14, 21, 38].

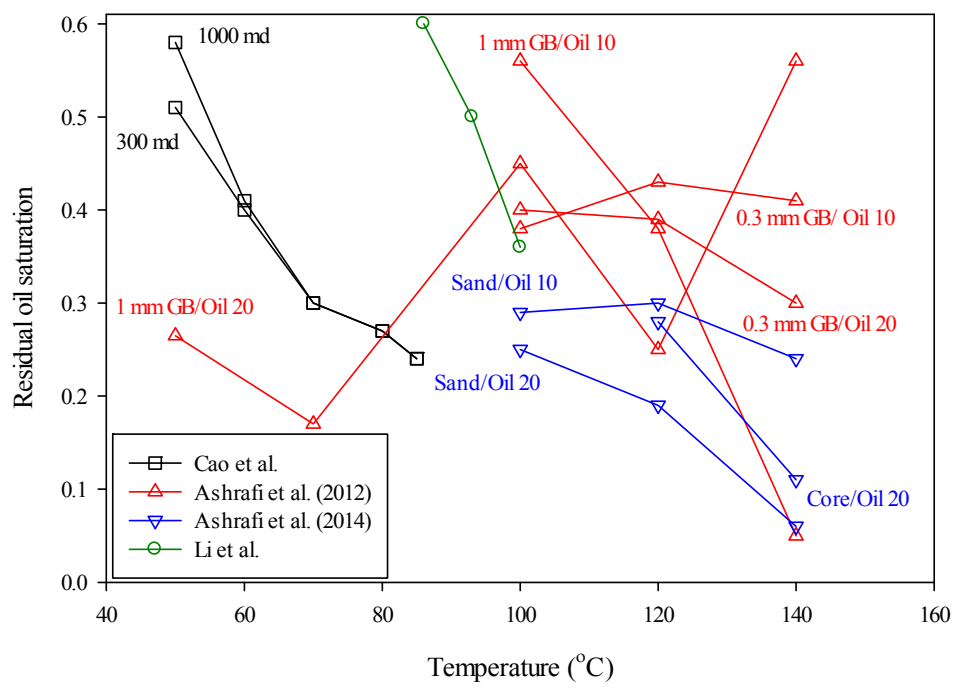

Figure 16. The variation of residual oil saturation for different porous media at elevated temperature [3, $4,45,94]$.

Vega et al. [18] used steady-state method for measuring oil/water relative permeability.

7 For pre-cleaning the core plug, a few pore volume of decane injection and gas flushing followed

8 by vacuum drying was used. CT-Scan confirmed a uniform water saturation distribution along the

9 core at each temperature. A nonlinear reduction of the residual oil saturation with increasing 
1 temperature was observed, as can be seen in Figure 18. The capillary end effect was also examined

2 in this study [18] by injecting water at $\mathrm{f}_{\mathrm{w}}=1$ with different flow rates and no variation in relative

3 permeability characteristics were observed. In another study, Zhang et al. [25] determined the

4 residual oil saturation by injecting $30 \mathrm{PV}$ of water into sandstone core plugs and found that Sor

5 decreased by increasing the temperature.

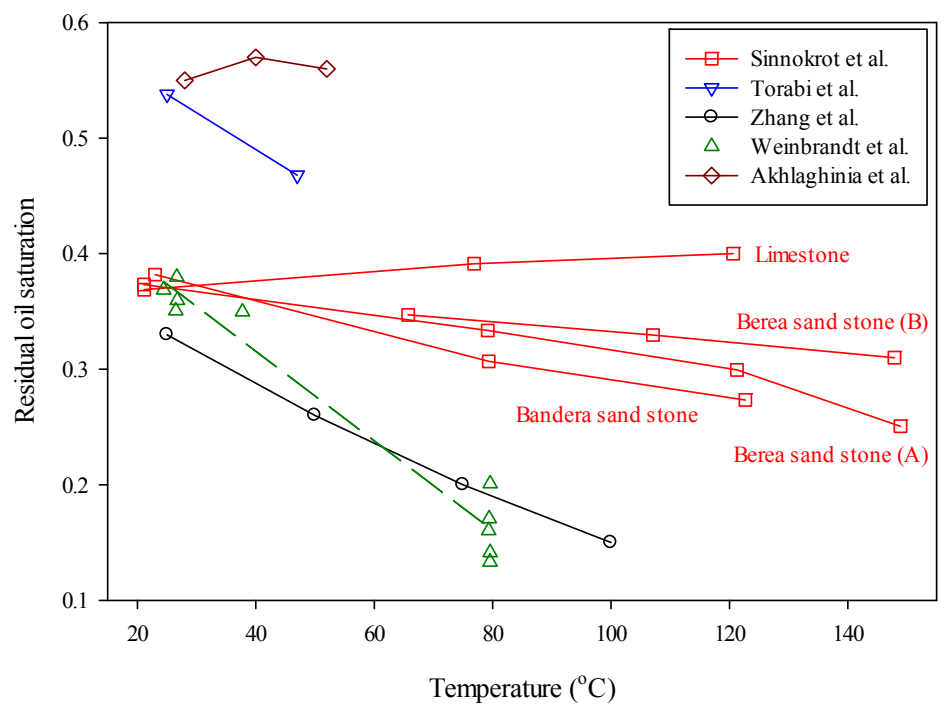

Figure 17. Different trends of residual oil saturation at higher temperatures suggested by some researchers $[2,20,23,25,42]$.

It should be mentioned that in viscous oil systems, it is necessary to inject copious volume of water to achieve the true residual oil saturation and it is generally not practical to continue the waterflood to this extent $[15,26]$. Different criteria have been used by researchers to terminate the waterflood. For example, Wang et al. [52] used the value of $99.9 \%$ water cut as the criterion to achieve the residual oil saturation in their experiments. $15 \mathrm{PV}$ of the water injection was used by Akhlaghinia et al.[2]. Weinbrandt et al. [20] determined the residual oil saturations by injecting 10 PV of water. Unfortunately, such criteria do not ensure that the oil saturation is close to the true residual saturation when the oil viscosity is very high. For example, when the oil/water viscosity ratio is 10,000 , as would be the case with many Canadian heavy oils at room temperature, the 
1 water cut of $99.9 \%$ occurs when the oil relative permeability is still 10 times higher than the water

2 relative permeability! A relative permeability ratio based condition for assuming the oil saturation

3 to be close to residual, such as $\mathrm{kro}_{\mathrm{k}} \mathrm{k}_{\mathrm{rw}}<0.01$, would be more reliable. However, for a $10,000 \mathrm{cP}$

4 oil, this will require the water-cut to be below $1 \mathrm{ppm}$ and may require many hundreds of pore 5 volumes of water injection.

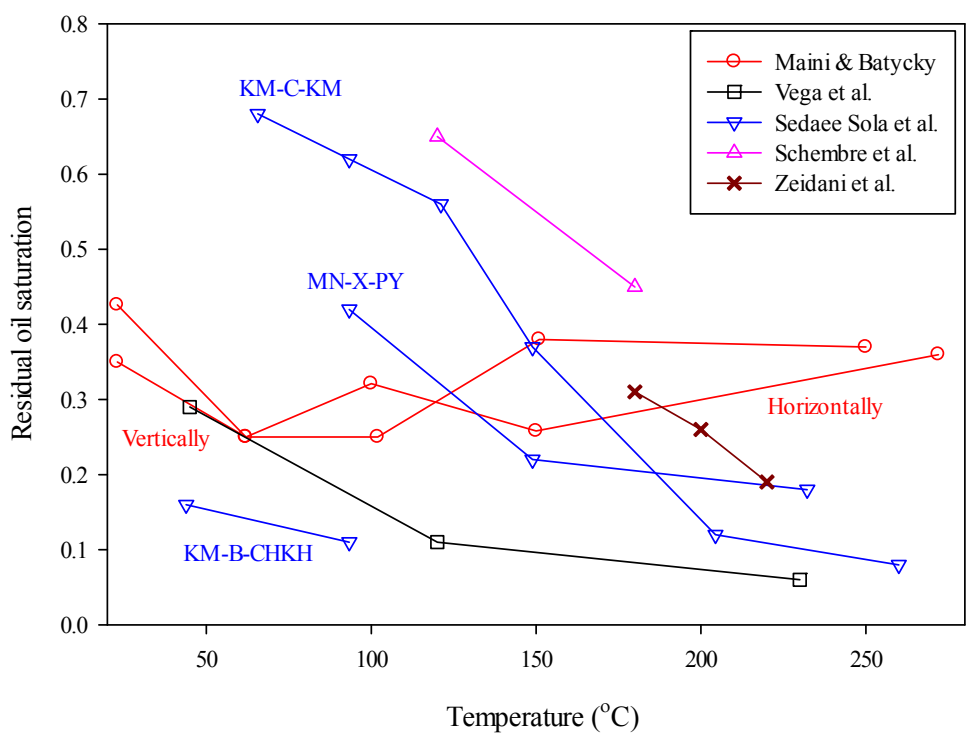

6

11 increased in diatomite core plugs. Frizzell's [46] developed a correlation, according to which the

12 residual oil saturation will decrease by increasing the temperature and decreasing the specific

13 gravity of oil. Their correlation shows that increasing the absolute permeability caused an increase

14 in the residual oil saturation, which they attributed to the higher tendency of water fingering

15 through the oil [46]. All the data employed in regression process were generated by injection of at

16 least $2 \mathrm{PV}$ of water or more until no oil was produced [46]. 


$$
S_{\text {or }}(\%)=-0.6394 \times\left({ }^{\circ} \mathrm{API}\right)-0.0619 \times\left({ }^{\circ} \mathrm{F}\right)+0.00128 \times k_{w}+51.15
$$

Another correlation was developed by Kumar et al. [40] by performing regression on the

2 data obtained by Torabzadeh et al. [24]. Kumar et al. [40] believed that for high tension system

3 (IFT $>1.0 \mathrm{mN} / \mathrm{m}$ ), the residual oil saturation was independent of IFT but decreased linearly with

4 increasing temperature. For low IFT systems, the residual oil saturation varied with both the

5 temperature and the IFT. These empirical correlations were stated as follows.

6 For high IFT:

$$
S_{o r}=0.3267-3.9477 \times 10^{-4} T
$$

7 For low IFT:

$$
S_{o r}=0.3267-3.9477 \times 10^{-4} T-2.825 \times 10^{-3} \sigma^{-2.0555}
$$

8 For ultra-low IFT:

$$
S_{\text {or }}=1.68639 \times(\sigma /(T+273.16))^{0.42342}
$$

They proposed another correlation based on the capillary number [46].

$$
S_{\text {or }}=0.2868-408.1\left[\tanh \left(N_{c}\right)\right]+1.925 \times 10^{5}\left[\tanh \left(N_{c}\right)\right]^{2}-2.663 \times 10^{7}\left[\tanh \left(N_{c}\right)\right]^{3}
$$
Hawkins [91], evaluated the residual oil saturation at ambient conditions and at the 11 reservoir temperature of $110{ }^{\circ} \mathrm{C}$ by injecting water until the water cut reached $99.9 \%$ and found 12 that the residual oil saturation did not change significantly [91]. Bennion et al. [38] found that the 13 residual oil saturation decreased with increasing temperature in hot water flooding. A cubic 14 correlation was fitted to the residual oil saturation data versus temperature $\left(\right.$ in $\left.{ }^{\circ} \mathrm{C}\right)$ during hot water 15 flooding in McMurray unconsolidated sand containing 8-9 ${ }^{\circ} \mathrm{API}$ bitumen, which is valid for the 16 temperature range between 10 and $280{ }^{\circ} \mathrm{C}$. 


$$
S_{\text {or }}=-5 \times 10^{-8} T^{3}+2.612 \times 10^{-5} T^{2}-5.16927 \times 10^{-3} T+0.6697519
$$

Sola et al. [17] determined the residual oil saturation during their relative permeability measurements when the oil fractional flow reached below $1 \%$. There was no change in the residual

3 oil saturation with a light oil (named $\mathrm{CHKH}$ oil) in the limestone core plug, while a reduction was

4 observed with heavy oil in both the dolomite and limestone core plugs [17]. The reduction of the

5 residual oil saturation displayed a linear trend in the experiments conducted under isothermal

6 conditions but a non-linear behavior was detected when the temperature was elevated 7 incrementally.

A reduction of the residual oil saturation from 0.54 to 0.47 was observed in the Torabi et

9 al. [42] study. Ashrafi et al. [3] also concluded that the residual oil saturation can be reduced at

10 higher temperatures. This reduction was attributed to the viscosity reduction and changes in 11 viscous fingering during core flooding tests.

In 1982, Sufi et al. [51] conducted an experimental study to investigate the effect of 13 temperature on oil/water relative permeability. The temperature was varied from 21 to $86^{\circ} \mathrm{C}$. They

14 concluded that the residual oil saturation decreased with increasing temperature. Furthermore, they 15 observed that by continuing the water injection after reaching $99 \%$ water cut, a slow reduction of 16 the practical residual oil saturation was seen. They suggested that the practical residual oil 17 measured at a preselected fractional flow of water (e.g. 0.99) might be temperature sensitive but 18 the true residual saturation, which can be obtained only by injecting an infinite amount of water, 19 would remain constant even at a higher temperature, as seen in Figure 19. They also found that the 20 flow rate had an effect on relative permeability and residual oil saturation at the rates below 4.166 $21 \mathrm{cc} / \mathrm{min}[51]$. Above this flow rate, the residual oil and oil relative permeability were independent 22 of the flow rate at room temperature [51]. 


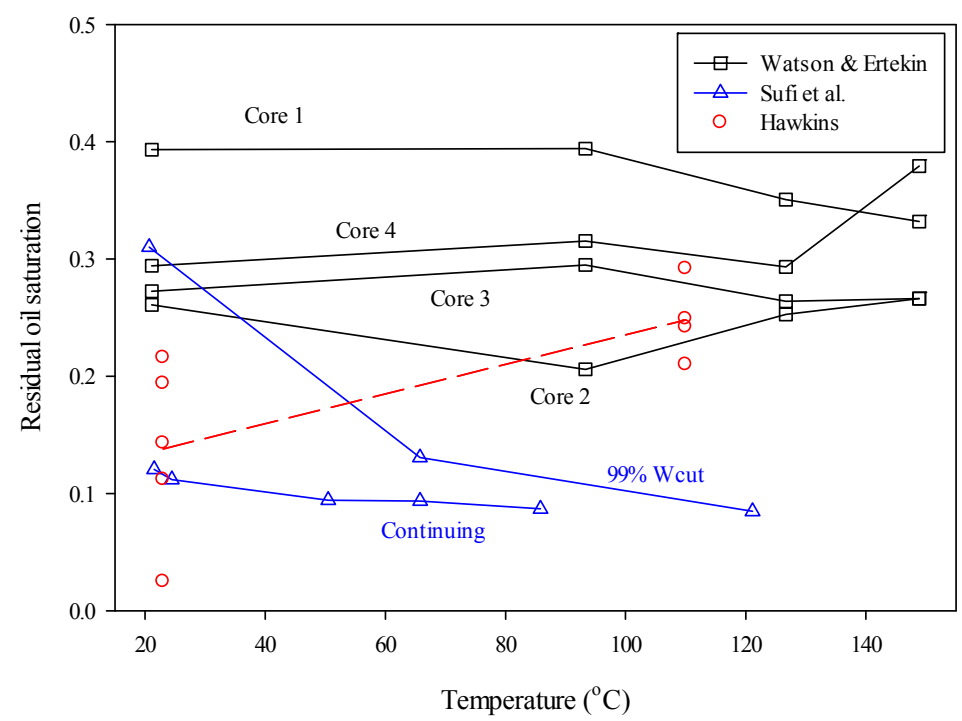

2 Figure 19. Comparison of the residual oil saturation variation with temperature increasing [19, 51, 91].

3 Miller and Ramey [90] used displacement tests to investigate the effect of temperature on

4 relative permeability. They found the residual oil saturation to be independent of temperature.

5 They mentioned that different results in this study compared to the previous studies were related

6 to the use of low viscosity light oil.

7 Polikar et al. [7] evaluated the effect of temperature on endpoint of relative permeability to

8 bitumen and water at elevated temperatures. The temperature was varied from 125 to $250{ }^{\circ} \mathrm{C}$ at a

9 constant pore pressure of $7 \mathrm{MPa}$. They asserted that the saturation and relative permeability 10 endpoint were apparently reached by pushing 4 to $5 \mathrm{PV}$ of brine into the sand-packs. Their results

11 showed that the temperature did not change the residual oil saturation significantly, as seen in

12 Figure 20. A small variation of the residual oil saturation with temperature was attributed to the

13 difference in sand-pack properties resulting from the packing procedure [7]. The effect of grain

14 size and brine composition were also investigated in this study using different mesh sizes and 15 adding $\mathrm{Na}_{2} \mathrm{SO}_{4}$ into the water phase, but no effect was detected. In another study, Polikar et al. [6] 
1 found that the residual bitumen saturation in unsteady-state measurements was not reached after 4

2 to $5 \mathrm{PV}$ injection and the recovery was enhanced by $18 \%$ after injecting more than $45 \mathrm{PV}$.

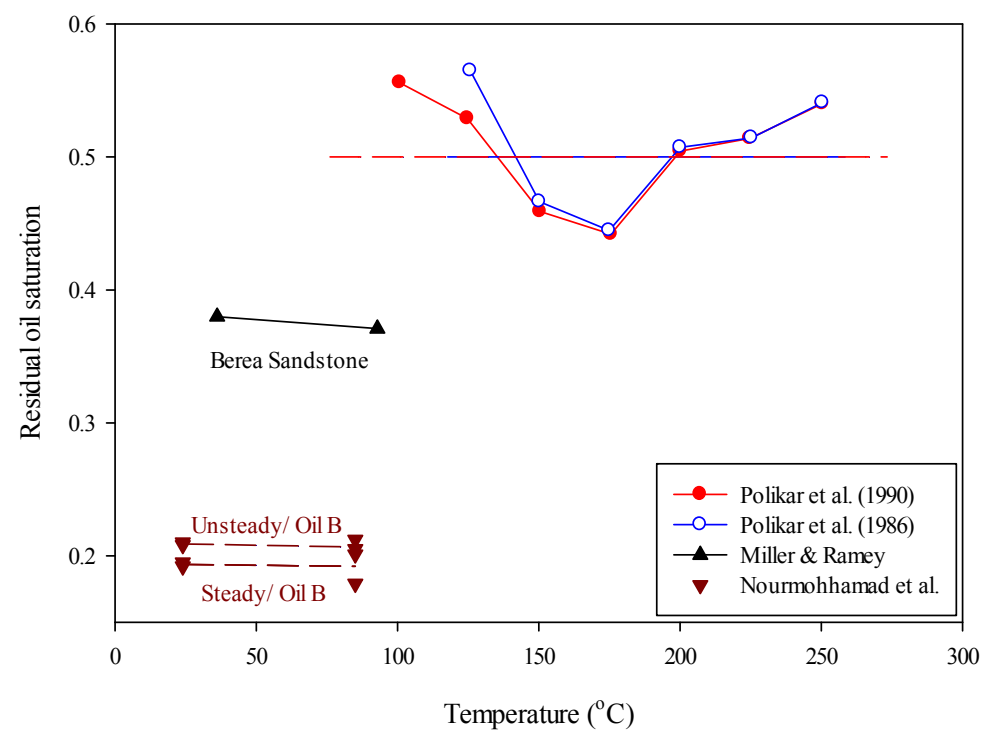

4 Figure 20. The residual oil saturation behavior at different temperatures reported by some researchers $[6$, $7,48,90]$. The temperature was found to have no direct impact on the residual oil saturation within a

7 temperature range of 24 to $85^{\circ} \mathrm{C}$ in a study performed by Nourmohammad et al. [48]. Wilson [86]

8 also observed the same result for the residual oil saturation. Watson \& Ertekin [19] also

9 demonstrated that the residual oil saturation was insensitive to the temperature in their

10 experimental tests. The residual oil saturation was taken as a fixed value equal to 0.2 in the history 11 matching process conducted by Maini \& Okazawa [8].

12 Kumar \& Inouye [47] injected more than 50 to 100 PV of water into the Berea sandstone 13 core plug or unconsolidated porous media to obtain the residual oil saturation when the fractional 14 flow of oil decreased below $0.1 \%$. They conducted three different experiments using different oils 15 at different temperatures and obtained similar results. The largest difference between water 16 saturation of two oils (White oil and silicon oil) occurred at the breakthrough time due to different 
1 wettability states [47]. The results of the residual oil saturation revealed that only the viscosity

2 ratio affected this parameter and it was independent of temperature [47].

3 In a study done by Akin et al. [37] in 1998, the same conclusion (no effect) about the

4 variation of the residual oil saturation with temperature was found. This study was conducted to

5 check the temperature impact on heavy oil and water system at a temperature range between 23

6 and $65.6{ }^{\circ} \mathrm{C}$. The experiments were performed at ambient temperature until no more oil was

7 produced, and the temperature was then increased. It was found that the temperature had a small

8 impact on the residual oil saturation in the applied temperature range [37]. This was attributed to

9 the unstable fluid flow detected by the CT-Scan technique. The value of the residual oil saturation

10 was around 0.12 at all temperatures. Esfahani et al. [12] conducted experimental measurements in

11 limestone and dolomite core plugs. To clean these cores prior to each experiment, toluene was

12 used as a solvent. After toluene flooding, all cores were placed in a furnace for 48 hours at a

13 temperature of $400{ }^{\circ} \mathrm{C}$. This study used only two different temperatures of $25^{\circ} \mathrm{C}$ and $105^{\circ} \mathrm{C}$. The

14 residual oil saturation in each core increased by about $2 \%$ at higher temperature. These values are

15 shown in Figure 21. Maini \& Batycky [15] used a different preserved core at each temperature and

16 found that the minimum value of the residual oil saturation was reached between 60 and $160{ }^{\circ} \mathrm{C}$.

17 Their residual oil saturation values are shown in Figure 18 and the inconsistent trends suggest that

18 the use of a different core in each test could have influenced the results [15]. Hamouda et al. [22]

19 also stated that the residual oil saturation first decreased from $42 \%$ to $36 \%$ at $80{ }^{\circ} \mathrm{C}$ and then

20 increased sharply up to $52 \%$ at $130{ }^{\circ} \mathrm{C}$ in a chalk core plug. Their results are shown in Figure 21 .

21 The preceding review of residual oil saturation reveals that most studies have measured

22 only the practical residual oil saturation after reaching some arbitrary threshold in terms of the

23 fractional flow of water or pore volumes injected. This practical residual oil saturation decreases 
1 with increasing temperature when a viscous oil is used, primarily because a high water-cut can be

2 reached with high viscosity oil even when the oil relative permeability is not close to zero. It is

3 also apparent that in clean systems, such as Ottawa sand or fired sandstone with refined oil and

4 water, the true residual saturation is independent of temperature. In real reservoir rocks with crude

5 oil and brine, the true residual oil saturation can also change with temperature due to changes in

6 wettability, interfacial tension and temperature induced changes in pore geometry. The direction

7 and extent of this change can only be determined experimentally. In absence of experimental

8 evidence to the contrary, the true residual oil saturation should be considered independent of 9 temperature.

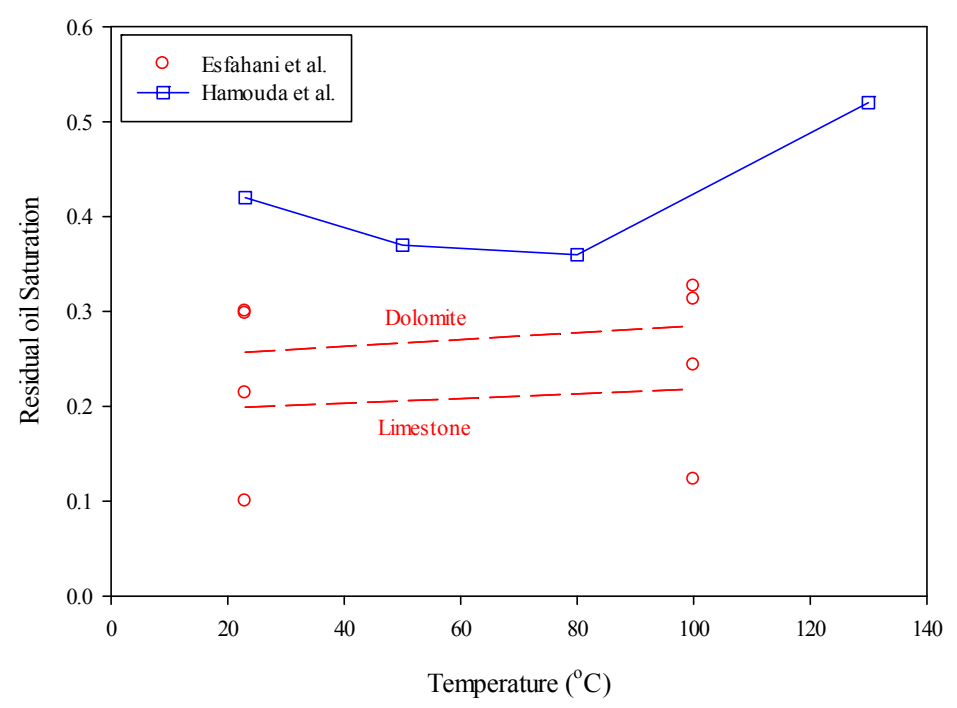

11 Figure 21. The value of residual oil saturation generated by Esfahani et al. [12] and Hamouda et al. [22].

12 For measurement of relative permeability in viscous oil systems, it would probably be

13 better to first conduct measurements at an elevated temperature that lowers the oil/water viscosity

14 ratio sufficiently to allow approaching the true residual oil saturation. The relative permeability

15 determined from this experiment can then be tested for history matching displacements conducted 
1 at lower temperatures. If a good history match of the lower temperature displacement can be

2 obtained, then the same relative permeability holds at the lower temperatures.

\section{6.3. Endpoint of relative permeability to water}

4 The endpoint relative permeability to water $\left(k_{r w}^{0}\right)$ is the water relative permeability at

5 residual oil saturation $[29,56]$, i.e. at the flood-out conditions when the oil is no longer produced

6 in a waterflood. Like the effect of temperature on other relative permeability parameters, such as

7 residual oil saturation or irreducible water saturation, there are conflicting results reported in the

8 literature on it also. Craig [83] showed that the value of water relative permeability at the residual

9 oil saturation is a good indicator of wettability. Accordingly, a change in wettability with

10 increasing temperature would be expected to cause a shift in the end point water relative

11 permeability; e.g. increased water-wetness would give lower $k_{r w}^{0}$. However, since the practical

12 residual oil saturation tends to decrease with increasing temperature, the measured water endpoint

13 shifts to higher water saturations at higher temperatures and this shift can increase the value of $k_{r w}^{0}$

14 (by extension of the curve to higher saturation) even when there is no change in wettability. Such

15 increase in $k_{r w}^{0}$ can mask the decrease related to increased water-wetness.

16 The range of temperature's effect on $k_{r w}^{0}$ becomes apparent from the reported results

17 presented in Figure 22 to Figure 26. Poston et al. [16] and Torabi et al. [42] reported an increase

18 in $k_{r w}^{0}$ with increasing temperature. Sola et al. [17] also found an increase in $k_{r w}^{0}$ with temperature

19 in a limestone core plug using a $26^{\circ} \mathrm{API}$ oil but with a higher viscosity oil, they found no change

20 in the endpoint in a dolomite core. Also, the temperature did not have any effect on the absolute

21 water permeability due to the total absence or very small content of clay in dolomite or limestone 
1 rocks [17]. A small change of the water endpoint relative permeability was detected by Li et al.

$2[94]$ where the endpoint increased by 0.04 at a higher temperature.

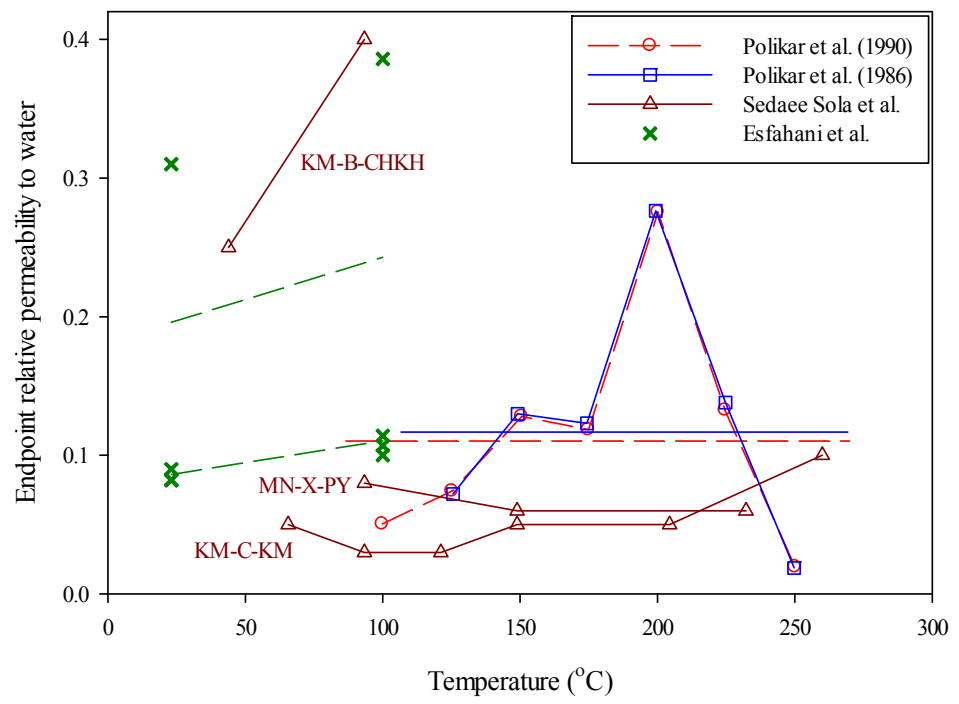

Figure 22. The water endpoint relative permeability at different temperatures reported by some researchers $[6,7,12,17]$.

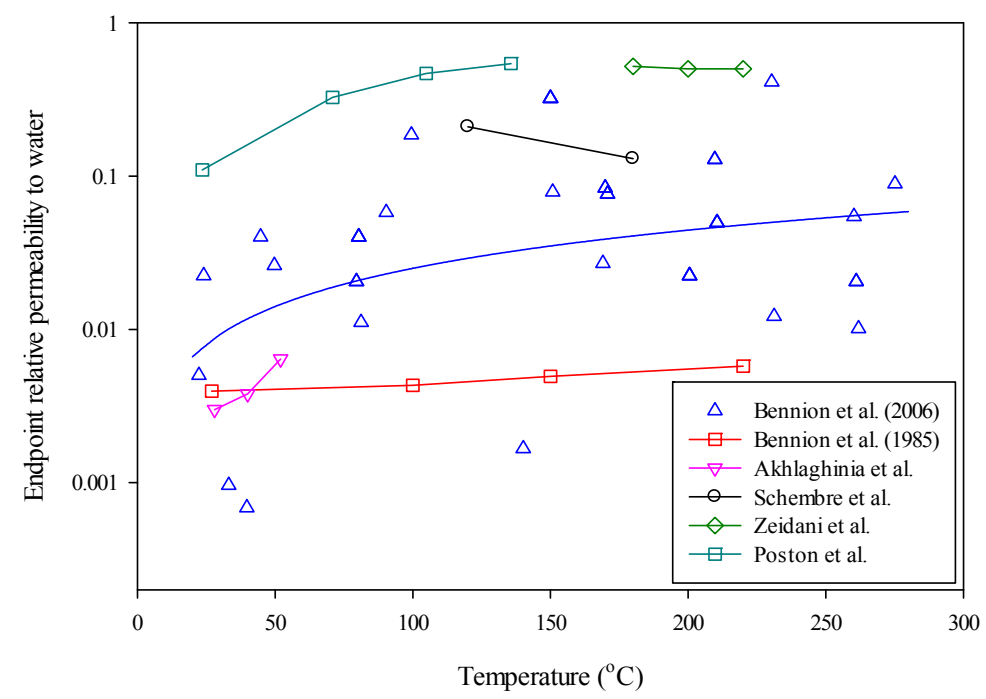

7 Figure 23. Different trends of water endpoint relative permeability at higher temperatures $[2,16,38,44$, 8 $50,53]$. 


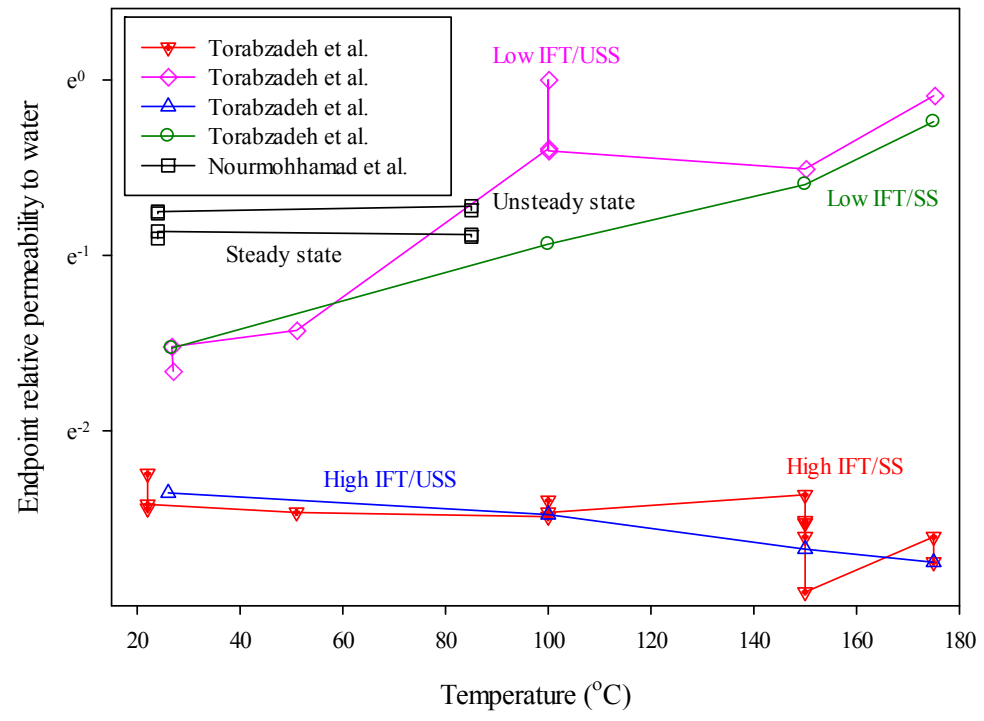

Figure 24. The variation of endpoint relative permeability to water for different systems [24, 48].

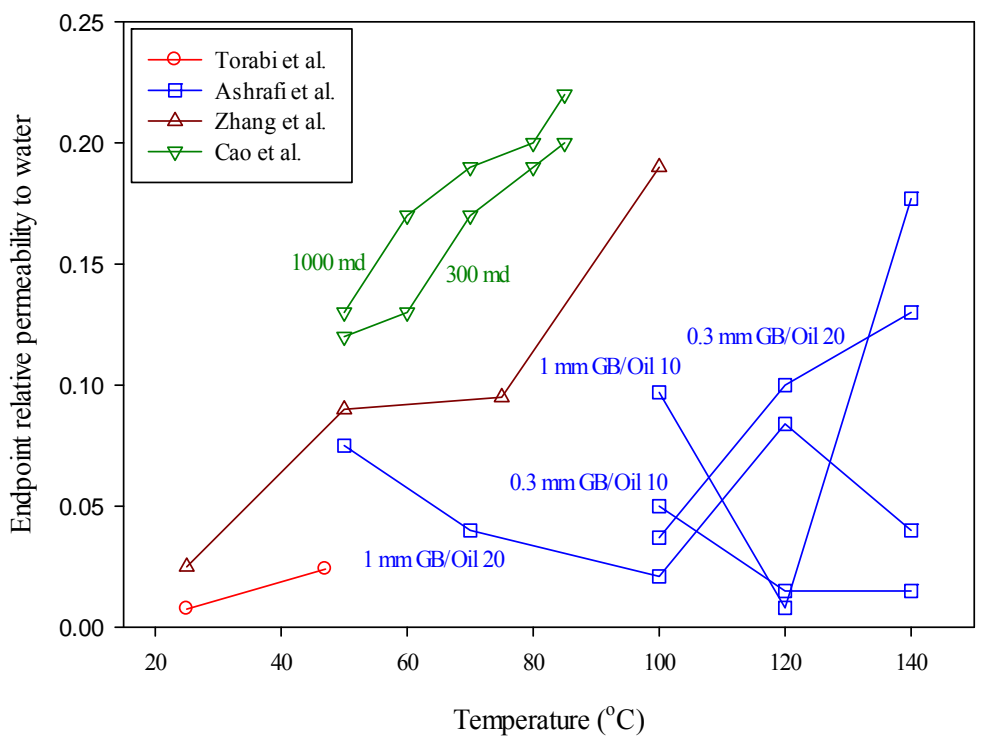

$4 \quad$ Figure 25. The changing in water endpoint relative permeability at higher temperatures [3, 25, 42, 45].

5 Bennion et al. [38,44] showed an increase in $k_{r w}^{0}$ when the temperature was raised.

6 Torabzadeh et al. [24] also reported an increase by increasing temperature within a temperature

7 range of $22-175^{\circ} \mathrm{C}$. An opposite results was obtained by Vega et al. [18] where $k_{r w}^{0}$ decreased

8 with temperature. They postulated that the absolute permeability was reduced by an order of 
1 magnitude and the porosity was also decreased at higher temperatures [18]. Mechanical stress,

2 chemical, and transport-related mechanisms were some of the possible causes of this decrease in 3 absolute permeability [18].

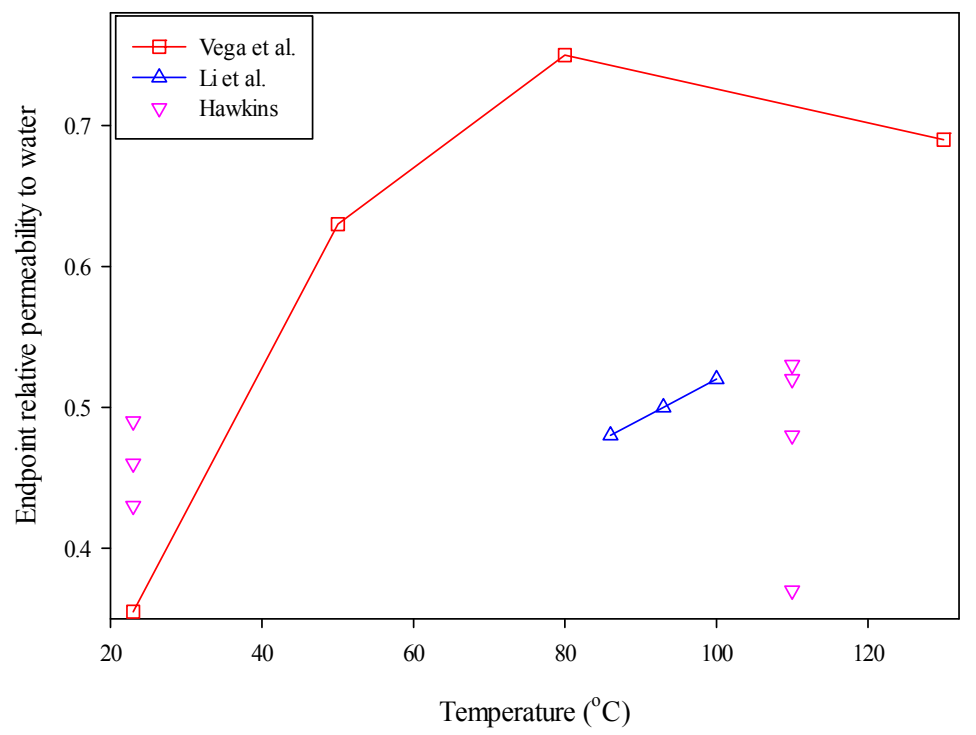

Figure 26. The comparison of the endpoint relative permeability to water with temperature increasing $[18,91,94]$.

Hawkins [91] compared the results of unsteady- and steady-state tests at reservoir

8 temperature $\left(110^{\circ} \mathrm{C}\right)$ with the steady-state results at the ambient temperature. They found that $k_{r w}^{0}$

9 was significantly lower in the unsteady-state measurements (compared to steady-state tests) at

10 ambient conditions [91]. Regarding the temperature impact, they suggested that the values of $k_{r w}^{0}$

11 at reservoir and ambient temperatures were essentially the same; as a result, they concluded that

12 the temperature did not affect the relative permeability to water at the residual oil saturation [91].

13 Torabi et al. [42] focused on eliminating the effect of physical properties of porous media

14 on two-phase relative permeability measurements at higher temperatures by performing the

15 experiments with the same core. After each test, the core was cleaned carefully and restored to its

16 initial wettability condition. The effect of pressure, flow rate, oil viscosity, and temperature were 
1 examined. All measurements were conducted within a limited temperature range of 27 to $45{ }^{\circ} \mathrm{C}$

2 and at pressure of 1.72 $\mathrm{MPa}$. In order to translate the production and pressure data of the unsteady-

3 state measurements to relative permeability curve, the JBN method [112] was employed. By

4 increasing either the pore pressure of the system from 1.72 MPa to $3.44 \mathrm{MPa}$ or decreasing the

5 temperature of the injected fluid, $k_{r w}^{0}$ decreased [42]. Furthermore, a reduction in $k_{r w}^{0}$ occurred

6 when the flow rate was increased or the oil phase viscosity was increased [42]. Torabi et al. [42]

7 suggested that in-situ oil emulsification during higher flow rate tests caused an improvement in

$8 k_{r w}^{0}$. However, the increase in $k_{r w}^{0}$ with the small increase in temperature was not explained.

9 Zhang et al. [25] recently conducted tests on the effect of temperature on two-phase relative

10 permeability. They used five different core plugs from a tight sandstone reservoir with strong

11 heterogeneities, a simulated oil containing $0.3 \%$ asphaltene, and distilled water containing $2.1 \%$

$12 \mathrm{KCl}$. The temperature remained constant in each experiment and a novel approach using a

13 combination of the JBN method [112] and Corey correlation [104] was used to convert the

14 production and pressure drop data into the oil and water relative permeability. They concluded

15 that $k_{r w}^{0}$ increased with increasing temperature [25]. The wettability alteration occurred in this

16 study was attributed to desorption of some polar components from the rock surface [25].

Polikar et al. [7] concluded that the water endpoint relative permeability was temperature

18 independent at temperatures lower than $200^{\circ} \mathrm{C}$. However, Polikar et al. [6] also suggested that $k_{r w}^{0}$

19 at higher temperatures could have changed due to some experimental difficulties under high

20 temperature condition. The system was water-wet based on the respective magnitude of relative

21 permeability at endpoints [6]. The effect of sand-grain size using 100/120 and 20/45 mesh sizes

22 was also investigated. Two different grain sizes with widely different permeability but same 
1 porosity were used and the measured endpoints showed similar results for both sand-grain sizes.

2 Furthermore, different overburden pressures resulted in the same value of endpoint.

3 Watson and Ertekin [19] conducted experiments at temperatures between room

4 temperature and $149{ }^{\circ} \mathrm{C}$. Their experimental results showed that $k_{r w}^{0}$ decreased with increasing

5 temperature. They mentioned that the reduction of $k_{r w}^{0}$ might be due to the apparent wettability

6 alteration by increasing temperature [19]. However, based on the observed reduction of both water

7 and oil relative permeabilities, they could not conclude that the wettability was changed.

8 Therefore, they suggested that any judgment about wettability alteration based on the value of the

9 irreducible water saturation or endpoint of oil and water relative permeability hypothesized by

10 Craig's rule [83] is not reliable and contact angle measurements are needed to confirm the

11 wettability change. Their values of endpoint relative permeability to water are shown in Figure 27

12 at different temperatures.

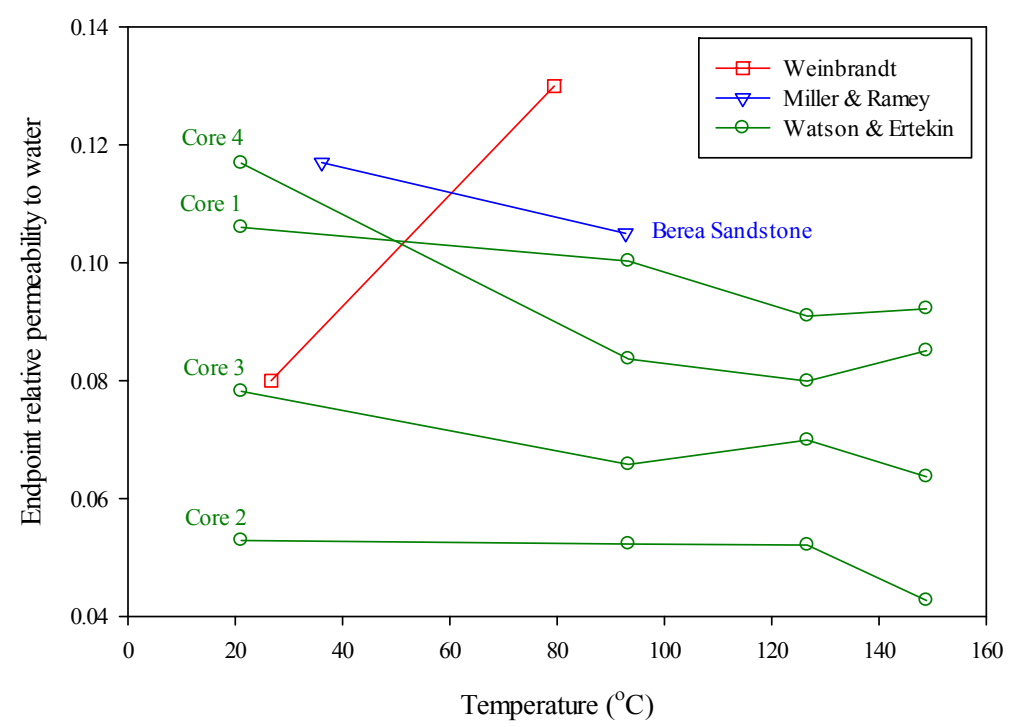

14 Figure 27. The different trends of endpoint relative permeability to water versus temperature [19, 20, 90]. 
Maini \& Batycky [15] claimed that $k_{r w}^{0}$ was generally not modified with increasing

2 temperature. Maini \& Okazawa [8] reported that $k_{r w}^{0}$ increased from 0.085 to $1.50 \mu \mathrm{m}^{2}$ during

3 temperature increase of approximately $180{ }^{\circ} \mathrm{C}$. In contrast Miller \& Ramey [90] showed that $k_{r w}^{0}$

4 remained constant at high temperatures. They also mentioned that the temperature did not have an

5 effect on water absolute permeability in the unconsolidated sand, while this value was reduced

6 permanently from $310 \mathrm{md}$ to $190 \mathrm{md}$ as temperature increased up to $93{ }^{\circ} \mathrm{C}$ in a consolidated Berea

7 sandstone core. No modification was observed by Nourmohammad et al. [48], Wilson [86], and

8 Sufi et al. [51]. However, a significant variation of $k_{r w}^{0}$, showing both increasing and decreasing

9 trends, with temperature was reported by Ashrafi et al.[3] in clean glass-bead packs. A significant

10 reduction of $k_{r w}^{0}$ at higher temperatures was recorded by Schembre et al. [50] in diatomite cores.

11 An empirical correlation for water relative permeability at the residual oil saturation was

12 developed by Frizzell [46], which is expressed as Eq. (12). An increase in the endpoint relative

13 permeability is expected at higher temperatures from this correlation [46].

$$
\begin{aligned}
& k_{r w}\left(S_{o r}\right)=\exp \left[-2.905+0.08927 \times\left({ }^{\circ} A P I\right)+0.0012 \times\left({ }^{\circ} F\right)+0.9565 \times \ln \left(k_{w}\right)\right. \\
& \left.-0.0255 \times\left(S_{w i}\right)\right]
\end{aligned}
$$

14 Hamouda et al. [22] found that the water endpoint relative permeability increased in going 15 up to $80{ }^{\circ} \mathrm{C}$ (from room temperature) and then it decreased slightly at $130{ }^{\circ} \mathrm{C}$. The change was 16 attributed to wettability alteration and decrease of absolute permeability with increasing 17 temperature [22].

Although it is obvious from the foregoing that the effect of temperature on endpoint water 19 relative permeability is different in different rock-fluid systems, some generalized observations 
1 can be made for thermal recovery of heavy oils. The review suggests that the variation of $k_{r w}^{0}$ with

2 temperature is not related to the rock cementation. However, carbonate rocks display greater

3 tendency of changes in $k_{r w}^{0}$ with temperature. The heavy oil viscosity changes dramatically with

4 increasing temperature, which results in a significant reduction of the practical residual oil

5 saturation. Recall that the residual oil saturation is typically measured by continuing the

6 displacement until the oil-cut becomes lower than a threshold value (e.g. 0.01 or 0.001 ). The

7 residual oil saturation measured at such threshold oil-cut will decrease with increasing temperature

8 even when there is no change in relative permeability and this will move the endpoint to higher

9 water saturation, resulting in higher $k_{r w}^{0}$. Therefore, in absence of any change in wettability and

10 interfacial tension, the endpoint relative permeability to water measured at a set fractional flow

11 threshold should be expected to increase with temperature. In systems that involve a significant

12 change in wettability, its impact will be superimposed on the effect of reduced residual oil

13 saturation due to increased mobility of the oil. Generally, the wettability appears to shift towards

14 increased water-wetness, which would tend to reduce the endpoint relative permeability to water.

15 In many cases, the impact of increased water-wetness counterbalances the impact of decreased

16 residual saturation and results in no significant change in end-point water relative permeability.

\section{$17 \quad 6.4$. Endpoint of relative permeability to oil}

The endpoint of relative permeability to oil $\left(k_{r o}^{0}\right)$ is the value of oil relative permeability at

19 the highest attainable oil saturation when oil displaces water [29, 56]. Often, the connate water

20 saturation in typical reservoirs is equal to the irreducible water saturation and the initial relative

21 permeability to oil is this endpoint value. Previous studies $[29,56]$ have reached a consensus that

22 the endpoint of relative permeability to oil phase depends primarily on the wettability state of the 
1 rock surface. Based on Craig's rule [83], $k_{r o}^{0}$ is an indicator of the wettability state of the rock

2 surface and higher values correspond to more water-wet systems. In a strongly water-wet system,

3 the irreducible water saturation would exist as a wetting film on pore surfaces of oil filled pores,

4 or as pendular rings at grain contacts and perhaps as pore filling fluid in very small pores [57-60].

5 This water saturation causes little or no interference to flow of oil and therefore almost full

6 conductivity of the porous medium becomes available to the oil. It means that $k_{r o}^{0}$ would be

7 expected to be close to $100 \%$ in strongly water-wet systems [56-58]. If the increase in temperature

8 makes the system more water-wet, $k_{r o}^{0}$ should be expected to increase, but if its value was already

9 close to $100 \%$, there would be little room left for such increase. Moreover, if the irreducible water

10 saturation becomes larger at higher temperatures, there could be greater interference in the flow of

11 oil due to the presence of water and a reduction in $k_{r o}^{0}$.

12 By reviewing previous studies done during the past sixty-five years, no firm conclusion

13 about the effect of temperature on $k_{r o}^{0}$ could be extracted. Experimental studies have reported a

14 full range of possibilities. Wilson [86] found that $k_{r o}^{0}$ remained constant. Poston et al. [16] also

15 found $k_{r o}^{0}$ to be independent of temperature. The end point to the oil phase at different temperatures

16 was reported equal to the unity in all cases but no further explanations as to reasons for this were

17 offered [16]. In another study done by Sufi et al. [51], the results clearly showed that $k_{r o}^{0}$ was not

18 a function of temperature and it was equal to unity at different temperatures. Maini \& Okazawa

19 [8] had similar opinion about the oil relative permeability at irreducible water saturation. They

20 demonstrated that, in clean unconsolidated sand, the effective permeability to oil at irreducible

21 water saturation remained constant at different temperatures. 
Miller and Ramey [90] investigated the effect of temperature on relative permeability for

2 consolidated Berea sand stone. The oil and water flood behaved almost as piston-like

3 displacements, therefore the relative permeability curve could not be determined and only the

4 endpoints of relative permeability values were obtained [90]. They concluded that within the

5 accuracy of measurements, the endpoint was independent of temperature.

6 Several other scholars have also reported no dependency of $k_{r o}^{0}$ with temperature.

7 Schembre et al. [50] performed experimental tests with unsteady-state approach to measure the

8 transient relative permeability of two phases with time. All spontaneous countercurrent water

9 imbibition tests were conducted at a temperature range between 120 and $180{ }^{\circ} \mathrm{C}$ and Simulated

10 Annealing method was applied to estimate the instantaneous relative permeability and capillary

11 pressure curve under unsteady-state conditions [50]. Their conclusion was that $k_{r o}^{0}$ does not change

12 significantly with temperature. Sola et al. [17] showed different results about the oil relative

13 permeability at irreducible water saturation. In their work, prior to each experiment, the core was

14 completely washed with toluene for three days and dried for at least 24 hours at the temperature

15 of $148.8^{\circ} \mathrm{C}\left(300^{\circ} \mathrm{F}\right)$ to remove all organic material from the rock surface. The results generated

16 during experiments with heavy oils named "PY" and "KM" on limestone and dolomite confirmed

17 that temperature did not change $k_{r o}^{0}$, while the increase in temperature in the system containing a

18 medium oil named "CHKM" in limestone core shifted the relative permeability endpoint from 1.0

19 at $37.8^{\circ} \mathrm{C}$ to 0.2 at $93.3{ }^{\circ} \mathrm{C}$ [17]. As a result, they suggested that two different behaviors could be

20 obtained in the limestone core sample, depending on the oil used. A decrease of endpoint relative

21 permeability to oil with temperature was also reported by Vega et al. [18], as shown in Figure 28, 
1 as well as by Esfahani et al. [12] on carbonate core plugs. Results of Watson and Ertekin [19],

2 shown in Figure 29, also reveal a similar trend.
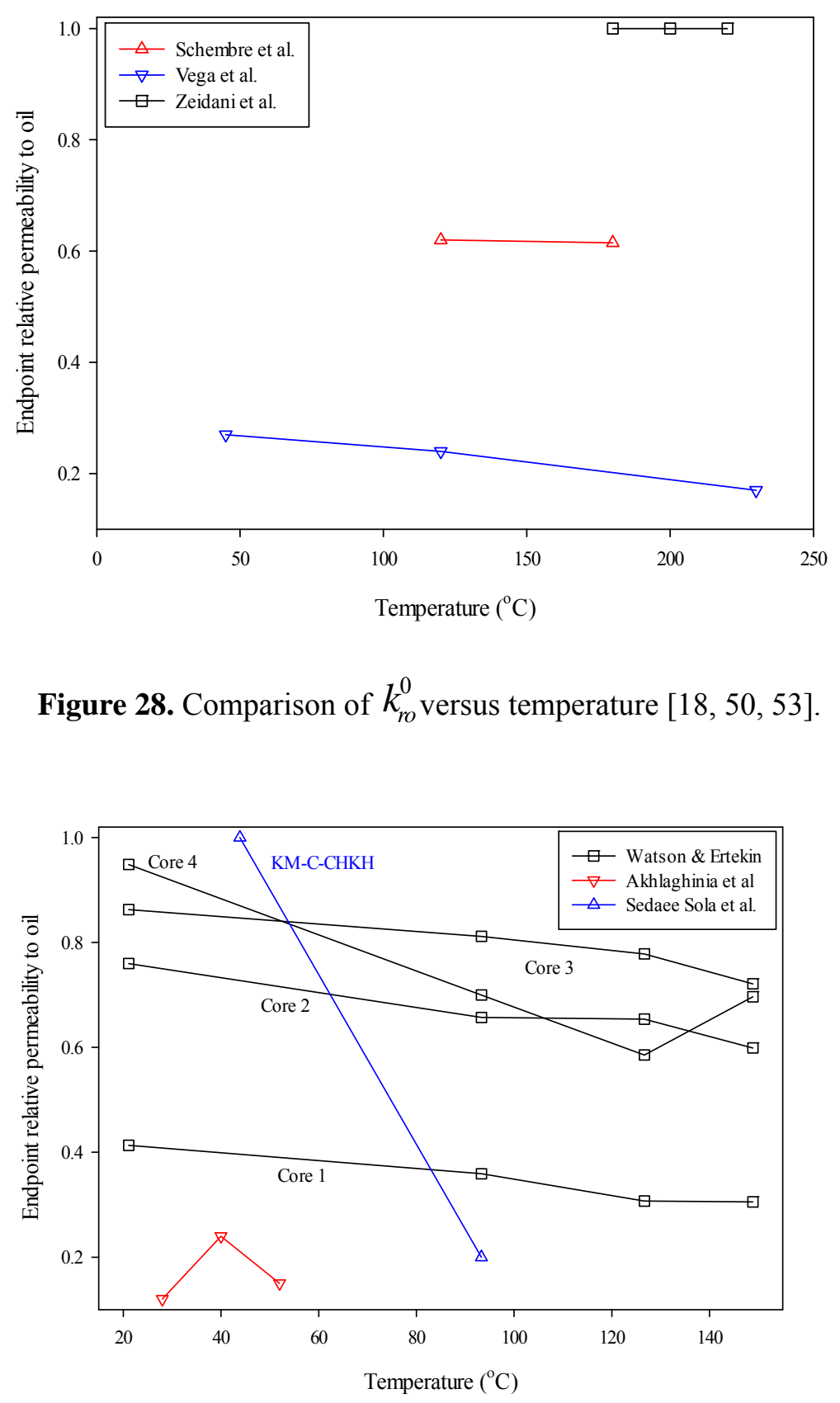

Figure 29. The behavior of endpoint relative permeability to oil versus temperature $[2,17,19]$. 
1 They concluded that the temperature had a small effect on absolute permeability of the clean,

2 unconsolidated Ottawa sand packs [7]. The results of these studies are illustrated in Figure 30.

3 Polikar et al.[6] suggested that the clay movement was a possible reason for absolute permeability

4 change. Torabi et al. [42] also reported $k_{r o}^{0}$ to remain unchanged at higher temperatures.

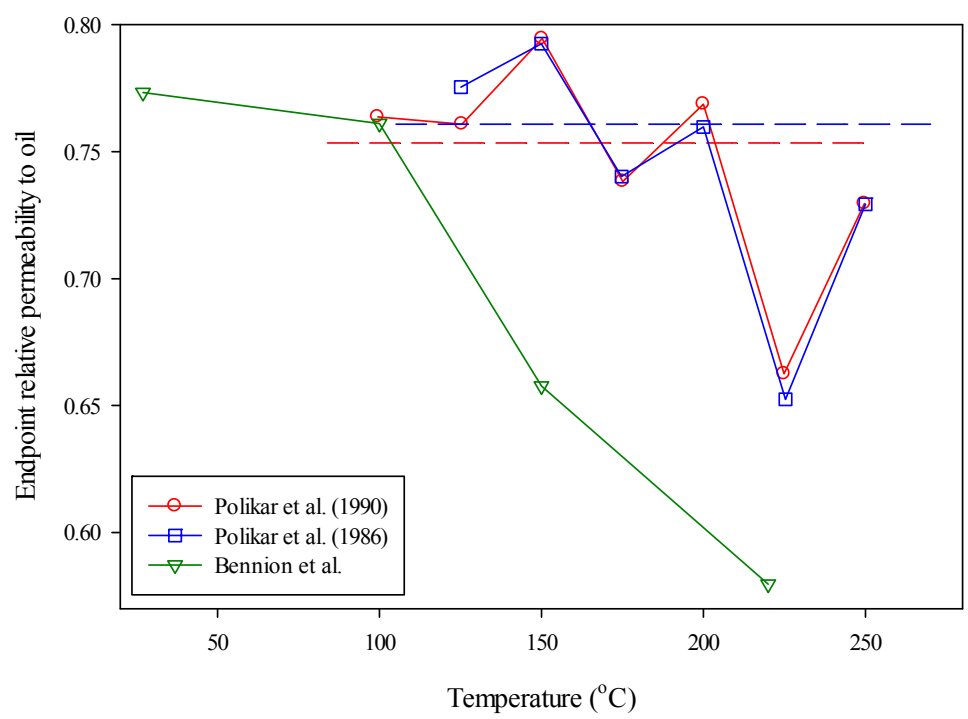

Figure 30. Different trends of oil endpoint relative permeability at higher temperatures $[6,7,44]$.

$8272{ }^{\circ} \mathrm{C}$. In their work, fluctuations were seen in the trend of $k_{r o}^{0}$ with temperature in horizontally

9 drilled cores, especially at $150^{\circ} \mathrm{C}$. However, a clear trend was seen for vertically drilled cores. The

10 endpoint declines in both horizontally drilled and vertically drilled plugs but the decline was more

11 pronounced in the latter [15]. The reduction in absolute permeability of core as well as the increase

12 in irreducible water saturation were suggested as a reason for this result. Moreover, it was

13 mentioned that the difference between horizontally and vertically drilled cores in $k_{r o}^{0}$ was caused

14 by the occurrence of very thin shale layers, thin siderite-cemented sand beds and other aspects of

15 the fluvial-meander-belt-type deposit [15]. 
Frizzell et al. [46] analyzed fifteen years of relative permeability measurements performed

2 by Texaco's Exploration and Production Technology Division. This study investigated the effect

3 of many factors (including temperature, absolute permeability and API gravity of oil) on residual

4 oil saturation, irreducible water saturation, effective permeability to oil and water. Regarding the

5 fluid properties and rock characteristics, crude oils and clean or restored unconsolidated sands

6 were used in these measurements. The test temperature varied from 24 to $204{ }^{\circ} \mathrm{C}$. It is notable that

7 during all experiments, researchers [46] ensured that the injection rate followed the criteria

8 proposed by Rapoport et al. [113] and they maintained the temperature constant in each test. Based

9 on their correlation, a downward trend for oil relative permeability endpoint with increasing

10 temperature is expected. The low value of the correlation coefficient of their correlation was

11 attributed to the effect of various artifacts. For example, the cleaning procedure, using the same

12 core in many experiments, different types of oil and effect of cyclically increasing temperature,

13 using a solvent to clean the core could have caused scattered data [46]. The correlation for endpoint

14 oil effective permeability is expressed as follow.

$$
\begin{aligned}
& k_{r o}\left(S_{w i}\right)=\exp \left[0.08974 \times\left({ }^{\circ} A P I\right)-0.00313 \times\left({ }^{\circ} F\right)+0.9561 \times \ln \left(k_{w}\right)-0.0018 \times\left(S_{w i}\right)\right. \\
& \left.-4.461 \times 10^{-1}\right]
\end{aligned}
$$

15 here $\mathrm{k}_{\mathrm{w}}$ is the absolute permeability to water in md and saturations are in percent.

In the study conducted by Bennion et al. [44], the temperature of the system was increased

17 incrementally during four stages from 27 to $220^{\circ} \mathrm{C}$, so when no more oil was produced at a given

18 temperature i.e. residual oil saturation was achieved, the temperature was increased to the next

19 higher level [44]. According to the reported results, the endpoint effective permeability to oil 
1 declined with temperature from $0.591 \mu \mathrm{m}^{2}$ at $27{ }^{\circ} \mathrm{C}$ to $0.414 \mu \mathrm{m}^{2}$ at $220{ }^{\circ} \mathrm{C}$. The values of $k_{r o}^{0}$ for

2 the preserved core plug are shown in Figure 30.

3 Although many studies $[12,15,17-19,40,44,46]$ have indicated the declining trend of oil

4 relative permeability endpoint with temperatures, Miller \& Ramey [90], Kumar et al. [40] and

5 Torabzadeh et al. [24] reported an increase in $k_{r o}^{0}$ at higher temperatures. In the study by Miller \&

6 Ramey [90], $k_{r o}^{0}$ failed to show any temperature dependency at early stages. However, the authors

7 reported an interesting phenomenon when they shut down the test for about an hour after ending

8 the experiments. Afterward, oil injection was restarted and the system was allowed to be stabilize.

9 The results showed that by restarting oil injection, the relative permeability to oil phase was

10 increased at irreducible water saturation [90]. The amount of such increase was larger at higher

11 temperatures [90]. Note that prior to shutting down the system, some water droplet were

12 periodically produced from the core outlet, while no water was drained from the core after oil

13 reinjection. The redistribution of water phase, collected near the production end due to capillary

14 end effect, caused this phenomenon during the shutdown period [90]. Miller \& Ramey believed

15 that this phenomenon will be enhanced as the fluid viscosity reduces at a higher temperature. This

16 temperature dependency is illustrated in Figure 31.

17 Torabzadeh et al. [24] investigated the effect of temperature in systems with different IFT

18 values. They indicated two different behavior of oil relative permeability endpoint with

19 temperature changes, as shown in Figure 31. They stated that the effect of wettability change and

20 IFT reduction at higher temperatures affected the endpoint in different directions. 


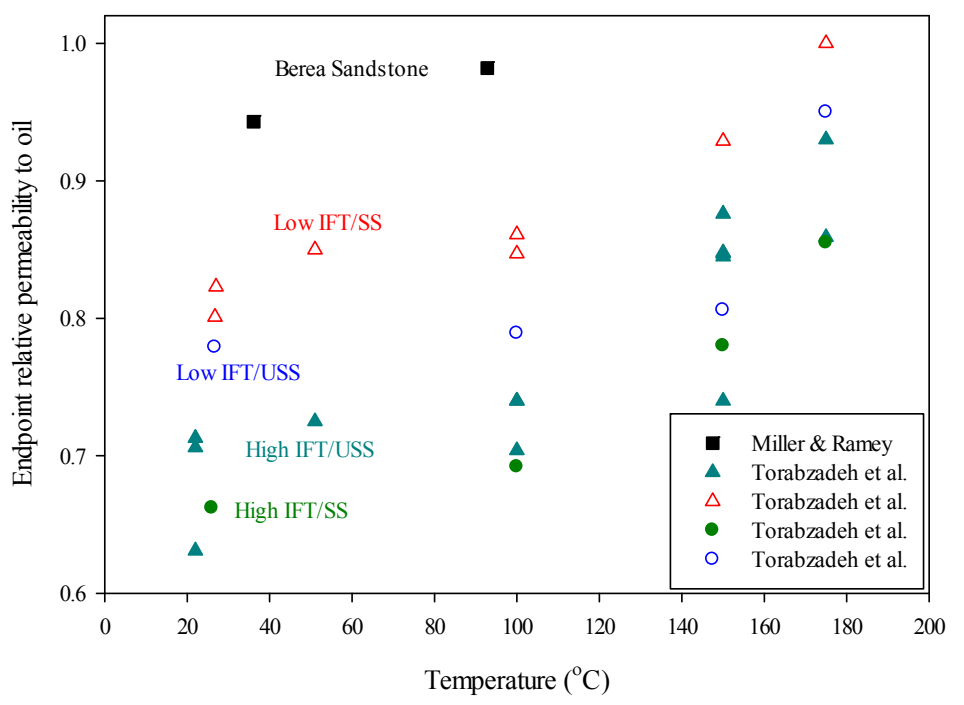

Figure 31. The variation of endpoint relative permeability to oil for different systems $[24,90]$.

Kumar et al. [40] developed empirical correlations to describe the temperature impact on

4 relative permeability characteristics based on the results of Torabzadeh et al. [24]. It was postulated

5 that for low tension system and high tension system, $k_{r o}^{0}$ increased and decreased with temperature

6 respectively [40]. The correlation developed for the oil endpoint relative permeability, which

7 applies to both systems, is shown in Eq. (14).

$$
k_{r o}\left(S_{w r}\right)=2.3136 \times S_{w i}^{0.49684} \times S_{o r}^{0.53469}
$$

8 The results generated by Ashrafi et al. [3], presented in Figure 32, show that the end point

9 fluctuated with temperature. Hamouda et al. [22] found that there is an optimum temperature at

10 which the highest endpoint can be achieved. However, Ashrafi et al. [3] did not completely agree

11 with this observation. They found that the behavior of endpoint relative permeability was case

12 dependent, which means that there might be an optimum temperature in some systems and not in 13 others. Hamouda et al. [22] found that at temperature of $85^{\circ} \mathrm{C}$, the endpoint reached 1.0 and then

14 decreased when temperature increased further. Some of the disagreement between these two 
1 studies is due to the use of different types of porous media, as Hamouda et al. [22] employed chalk

2 cores and Ashrafi et al. [3] used glass beads. Figure 32 depicts all data presented in these two

3 studies. Maini \& Okazawa [8] and Nourmohammad et al. [48] concluded that the endpoint oil

4 permeability was independent of temperature, and Akin et al. [37] stated that the temperature had

5 little or no impact on the endpoint of relative permeability to oil. Kumar \& Inouye [47] also

6 demonstrated that endpoint relative permeability was temperature independent but it depended on

7 the viscosity ratio.

8 14 by increasing temperature.

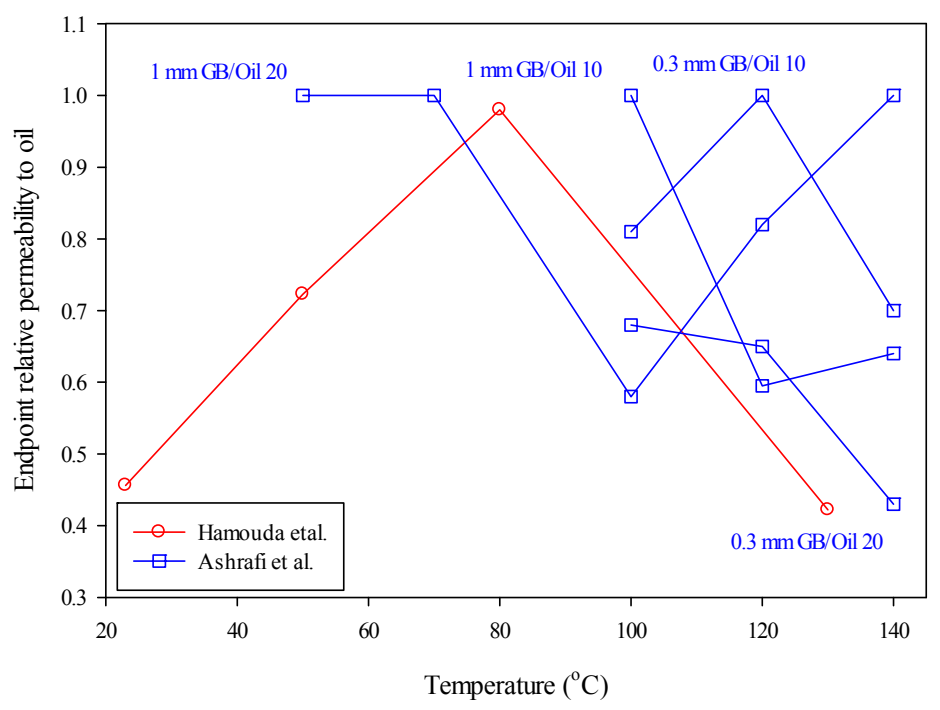

Figure 32. The changing in oil endpoint relative permeability at higher temperatures [3, 22].

Although, there are conflicting results in different systems, it appears that in majority of reported studies the endpoint relative permeability to oil was found to be independent of temperature. Therefore, unless reliable experimental information for a specific system shows a different trend, the endpoint relative permeability to oil should be assumed to remain unaffected 


\section{$1 \quad$ 6.5. Shape of water relative permeability curve}

Once the irreducible water saturation, the residual oil saturation, and the endpoint relative

3 permeability to water are known, the only thing remaining to determine in water relative

4 permeability is the shape of the curve. In some cases this shape can be quite complex and may

5 include points of inflection. However, when the wettability is uniform and the pore size

6 distribution is unimodal, the shape is expected to be relatively simple and it can be approximated

7 by the following equation [104].

$8 \quad k_{r w}=k_{r w}^{0}\left(S_{e}\right)^{n w}$

$S_{e}=\frac{S_{w}-S_{w i}}{1-S_{w i}-S_{o r}}$

9 The effect of temperature on the shape can be described by examining how the value of the

10 exponent $(n w)$ changes with temperature. This requires fitting the above equation to each reported

11 relative permeability curve to determine the exponent $(n w)$, unless the exponent value was already

12 reported in the paper $[4,17]$. This was done for the reviewed studies that reported a significant

13 effect of temperature on water relative permeability. In some cases the fitted values of exponents

14 were very large (greater than 6) or negative and these were considered outliers and discarded. The

15 remaining values are plotted against the temperature in Figure 33. It is apparent that there is no

16 easily discernable trend in this data. The linear regression line shows a small increase in $n w$ with

17 temperature starting from a value of 2.0 at the ambient temperature, but with very poor correlation

18 coefficient. Therefore, it can be suggested that in the absence of reliably measured values, the

19 shape of water relative permeability should be considered independent of temperature. 


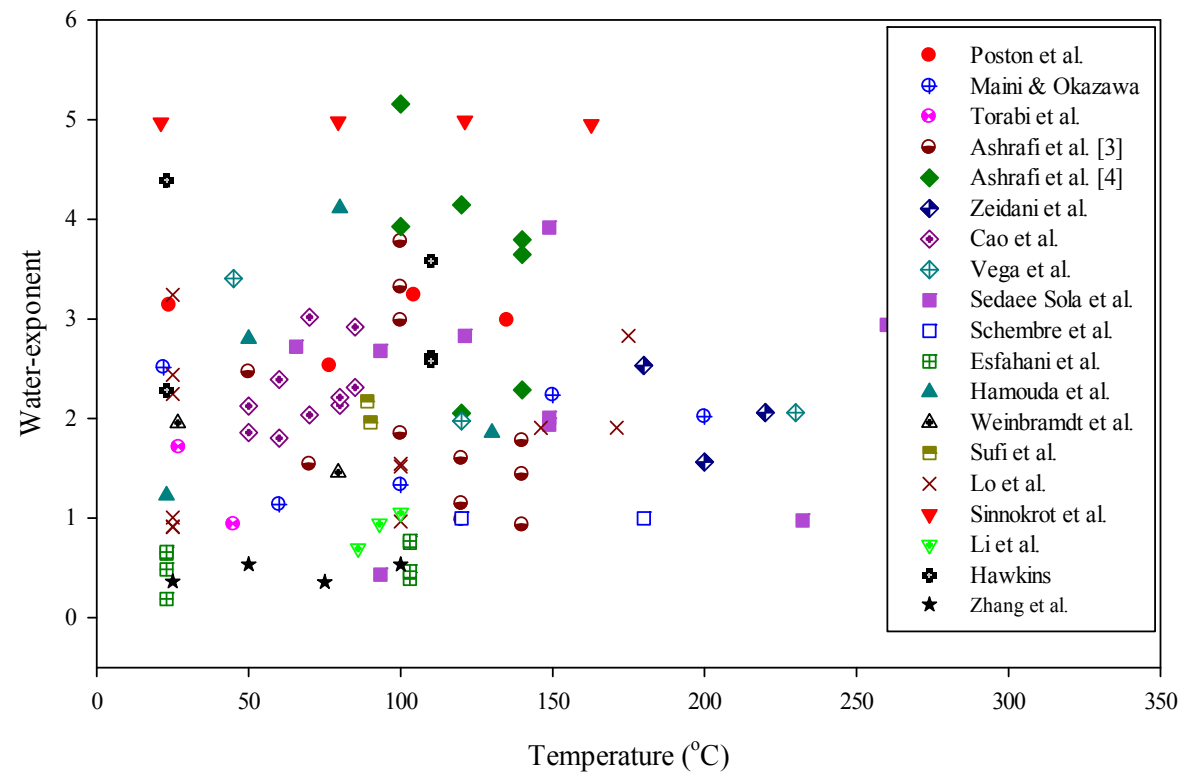

Figure 33. The effect of temperature on exponent of water relative permeability.

\section{6.6. Shape of oil relative permeability curve}

The shape of oil relative permeability was examined the same way as water relative

5 permeability curve discussed above. In majority of the reported cases, it can be approximated by

6 the following equation [104].

$$
k_{r o}=k_{r o}^{0}\left(1-S_{e}\right)^{n o}
$$

The effect of temperature on the shape of relative permeability curve was examined by

8 determining how the best fit value of the exponent (no) changes with temperature. This required

9 fitting Eq. (17) to the reported relative permeability curves in studies that did not directly report

10 the value of the exponents. The results are shown in Figure 34. The exponent values higher than 6

11 and negative exponents were again considered outliers and discarded. The values show no clear-

12 cut trend with increasing temperature. Therefore, we conclude that in the absence of reliable

13 experimental data for the system of interest, the shape of oil relative permeability should also be

14 considered independent of temperature. 


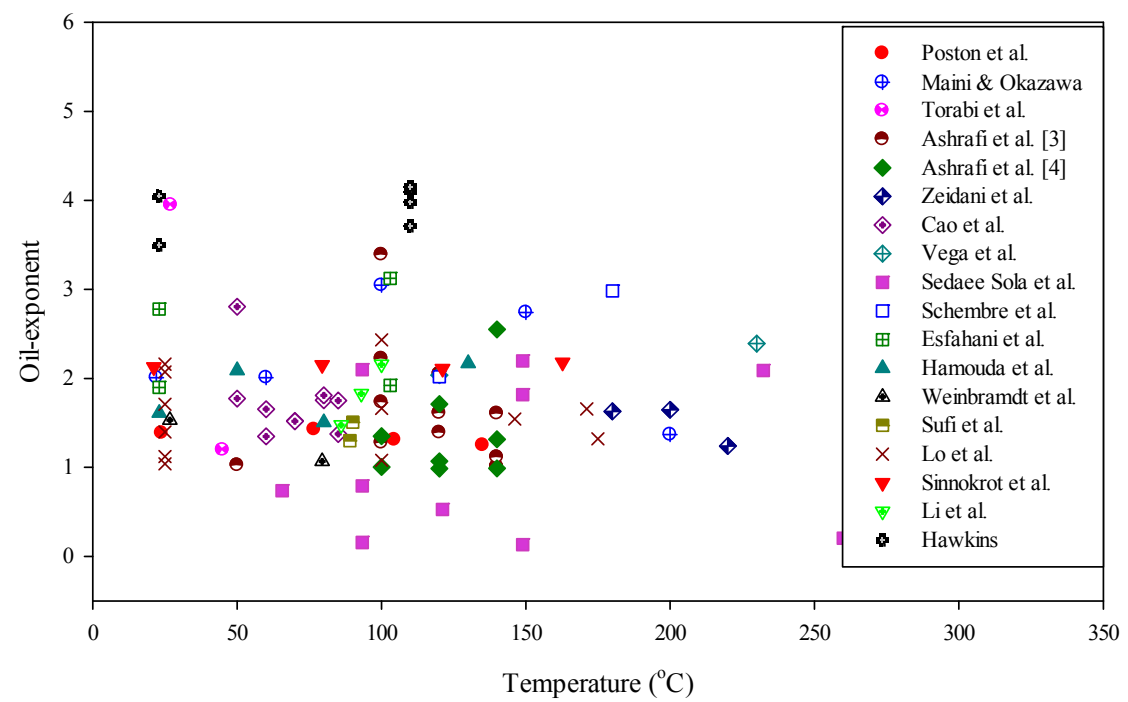

Figure 34. The effect of temperature on exponent of oil relative permeability.

\section{$3 \quad$ 6.7. Discussion}

Both steady-state and unsteady-state measurements suffer from experimental artifacts to

5 varying degrees. In steady-state tests, the principal sources of experimental errors are capillary

6 end-effects and uncertainties in saturation measurements. Large volumes of fluid injection are

7 needed to achieve the steady-state condition at each setting, which makes the determination of

8 saturation by material balance unreliable. In-situ saturation measurements of different type have

9 their own artifacts. However, the steady-state measurements with a reliable in-situ saturation

10 measurement technique would be relatively less prone to experimental artifacts. In unsteady-state

11 measurements, the artifacts include difficulties in maintaining accurate material balance to

12 determine the saturations, capillary end effects at the production end, premature termination of the

13 test before reaching close to the end-point saturation, and differences in the methodology used to

14 infer relative permeability from displacement data.

15 The problem of viscous fingering in displacement tests is a source of error in many studies.

16 As stated earlier, the relative permeability is a useful function of saturation only when capillary 
1 forces control the fluid distribution within the pore-space. This requires running the displacement

2 tests under conditions that will ensure a stable displacement, which often translates to using gravity

3 stable displacements at very low flow velocity. However, the need to minimize the capillary end

4 effects requires higher velocity displacements and in viscous oil systems, these two conflicting

5 requirements are difficult to reconcile.

6 Rapoport and Leas [113], and Peters and Flock [114] developed a scaling factor and a

7 dimensionless number respectively, which are useful in assessing the impact of capillary end

8 effects and viscous fingering in displacement tests. The effect of the scaling coefficient introduced

9 by Rapoport and Leas [113] on oil recovery at breakthrough time is shown in Figure 35. The oil

10 recovery is independent of the scaling coefficient, when its value is above $3.5 \mathrm{~cm}^{2} . \mathrm{cP} / \mathrm{min}$. The

11 Peters and Flock dimensionless number [114] is expressed in Eq. (18) through Eq. (20). As shown

12 in Figure 36, the unstable region consists of a transition zone, which occurs, in the dimensionless

13 number range of 13.56 and 1000 . Therefore, a stable displacement occurs when this factor is below

14 13.56. Note that the wettability number used in Eq. (18) is taken to be 5.45 for the oil-wet media

15 and 306.25 for the water-wet porous media [114].

$$
\begin{aligned}
& I_{s c}=\frac{(M-1)\left(v^{*}-v_{c}\right) \mu_{w} D^{2}}{C^{*} k_{\text {wor }} \sigma} \\
& v_{c}=\frac{k_{\text {wor }}\left(\rho_{w}-\rho_{o}\right) g \cos \alpha}{\mu_{w}(M-1)} \\
& M=\frac{\mu_{o} k_{\text {wor }}}{\mu_{w} k_{\text {oir }}}
\end{aligned}
$$

Only a small number of researchers paid attention to these numbers and ensured that

17 viscous fingering and capillary end effects did not affect their results. Often description of 18 experimental conditions is missing some of the parameters needed to calculate these numbers. In 
1 majority of the reported studies, flow velocity appears to be high enough to satisfy the scaling

2 coefficient of Rapoport and Leas [113] but in most of the displacements with viscous oils, the

3 stability number appears to be too high to ensure a stable displacement. Table 5 shows the fluid

4 flow velocity, injection flow rate or the viscous stability numbers reported in some studies.

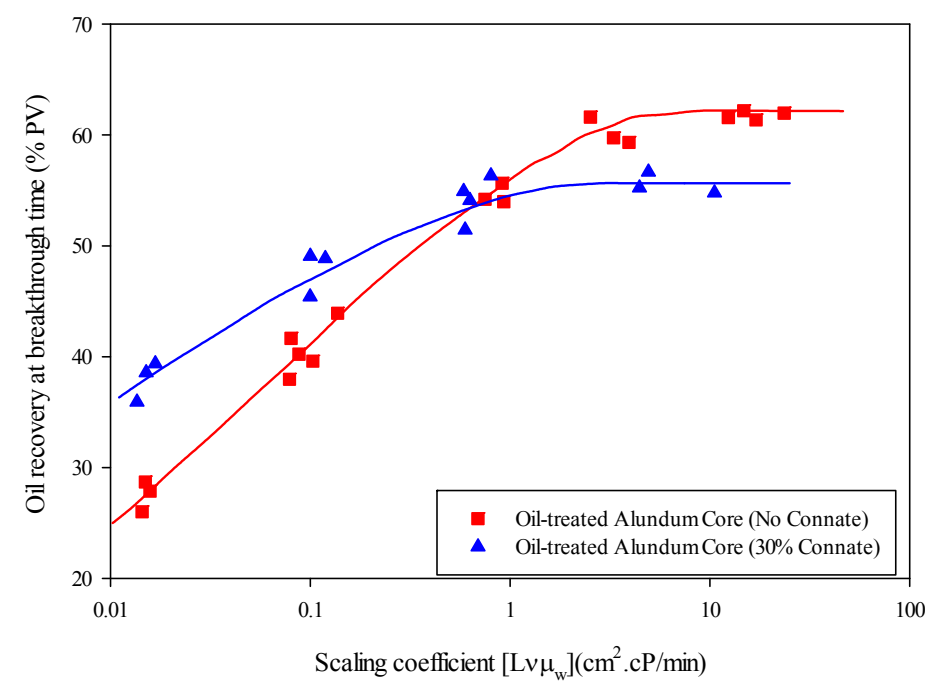

6 Figure 35. The recovery factor as function of the scaling coefficient at the breakthrough time [113].

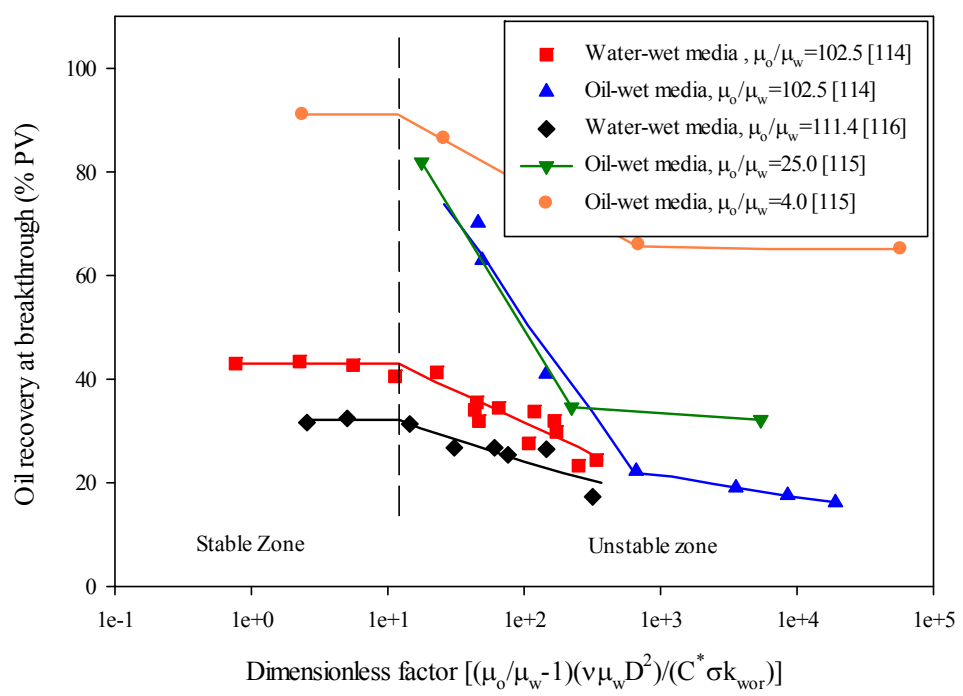

Figure 36. Recovery data versus the dimensionless parameter from the literature [114-116]. 
Finally, it is unreasonable to expect that the effect of temperature on oil-water relative

2 permeability would be same in all rock-fluid systems. The impact of temperature arises from

3 temperature-induced changes in wettability, interfacial tension and possibly pore geometry,

4 although the latter is not a factor in many systems. These changes depend on the initial wettability

5 condition and the mineral composition of the rock and the chemical composition of the fluids.

6 Generally, increased temperature reduces adsorption of species on rock surfaces and if the initial

7 wettability were influenced by adsorption of polar organic components, there would be a stronger

8 possibility of a change in wettability with increase in temperature. The effect of temperature on

9 interfacial tension will also be different depending on the composition of the oil and brine.

10 Therefore, it is not advisable to generalize the effect of temperature on oil-water relative

11 permeability.

12 Table 5. The value of fluid flow velocity or injection flow rate reported in the reviewed studies.

Fluid flow velocity or injection Flow rate

\begin{tabular}{|c|c|c|c|c|c|}
\hline Researcher & Year & Value & Researcher & Year & Value \\
\hline Wilson [86] & 1956 & $\begin{array}{c}0.0026-0.78 \mathrm{cc} / \mathrm{min} \\
\quad(\mathrm{ID}=2.587 \mathrm{~cm})\end{array}$ & Edmondson [21] & 1965 & $\begin{array}{c}7 \mathrm{cc} / \mathrm{min} \\
(\mathrm{ID}=5.09 \mathrm{~cm})\end{array}$ \\
\hline $\begin{array}{l}\text { Weinbrandt et al. } \\
\text { [20] }\end{array}$ & 1975 & $\begin{array}{l}0.359-0.891 \\
\mathrm{cc} / \mathrm{min}\end{array}$ & $\begin{array}{c}\text { Amaefule and Handy } \\
{[89]}\end{array}$ & 1982 & $\begin{array}{c}2.0 \mathrm{cc} / \mathrm{min} \\
\left(\mathrm{CA}=5.07 \mathrm{~cm}^{2}\right)\end{array}$ \\
\hline Sufi et al. [51] & 1982 & $\begin{array}{c}6.67 \mathrm{cc} / \mathrm{min} \\
(\mathrm{ID}=2.54 \mathrm{~cm})\end{array}$ & Torabzadeh et al. [24] & 1984 & $\begin{array}{l}0.5-3.0 \mathrm{cc} / \mathrm{min} \\
(\mathrm{ID}=2.54 \mathrm{~cm})\end{array}$ \\
\hline Polikar et al. [7] & 1986 & $0.9 \mathrm{PV} / \mathrm{hr}$ & $\begin{array}{l}\text { Watson and } \\
\text { Ertekinb[19] }\end{array}$ & 1988 & $\begin{array}{c}3.33 \mathrm{cc} / \mathrm{min} \\
(\mathrm{ID}=5.08 \mathrm{~cm})\end{array}$ \\
\hline $\begin{array}{c}\text { Closemann et al. } \\
{[84]}\end{array}$ & 1988 & $0-0.188 \mathrm{cc} / \mathrm{min}$ & Hawkins [91] & 1989 & $2.0-10.0 \mathrm{cc} / \mathrm{min}$ \\
\hline Polikar et al. [6] & 1990 & $6.6 \mathrm{ft} /$ day $(1 \mathrm{PV} / \mathrm{hr})$ & Kumar and Inouye [47] & 1994 & $\begin{array}{c}3 \mathrm{cc} / \mathrm{min} \\
(\mathrm{ID}=5.08 \mathrm{~cm})\end{array}$ \\
\hline Siddiqui et al. [92] & 1999 & $1.67 \mathrm{cc} / \mathrm{min}$ & Wang et al. [52] & 2006 & $0.167 \mathrm{cc} / \mathrm{min}$ \\
\hline
\end{tabular}


$(\mathrm{ID}=4.25 \mathrm{~cm})$

\begin{tabular}{|c|c|c|c|c|c|}
\hline $\begin{array}{c}\text { Sedaee Sola et al. } \\
\text { [17] }\end{array}$ & 2007 & $\begin{array}{c}\text { KM: } 0.003-0.557 \\
\text { PY: } 0.2-0.333 \mathrm{cc} / \mathrm{min} \\
(\mathrm{ID}=3.83 \mathrm{~cm})\end{array}$ & Ashrafi et al. [3] & 2012 & $\begin{array}{l}0.8-0.1 \mathrm{cc} / \mathrm{min} \\
(\mathrm{ID}=3.8 \mathrm{~cm})\end{array}$ \\
\hline $\begin{array}{c}\text { Akhlaghinia et al. } \\
\text { [2] }\end{array}$ & 2013 & $\begin{array}{c}0.1 \mathrm{cc} / \mathrm{min} \\
(\mathrm{ID}=2.54 \mathrm{~cm})\end{array}$ & Vang et al. [18] & 2014 & $\begin{array}{c}0.003-0.05 \mathrm{cc} / \mathrm{min} \\
(\mathrm{ID}=2.54 \mathrm{~cm})\end{array}$ \\
\hline Ashrafi et al. [4] & 2014 & $\begin{array}{c}0.5 \mathrm{cc} / \mathrm{min} \text { (oil) } \\
0.8 \mathrm{cc} / \mathrm{min} \text { (water) } \\
(\mathrm{ID}=3.8 \mathrm{~cm})\end{array}$ & Cao et al. [45] & 2014 & $\begin{array}{c}0.1 \mathrm{cc} / \mathrm{min} \text { (oil) } \\
0.1-0.5 \mathrm{cc} / \mathrm{min} \text { (Water) } \\
(\mathrm{ID}=2.427-2.518 \mathrm{~cm})\end{array}$ \\
\hline $\begin{array}{c}\text { Nourmohammad et } \\
\text { al. [48] }\end{array}$ & 2015 & $\begin{array}{c}9 \mathrm{cc} / \mathrm{min} \\
(\mathrm{ID}=3.81 \mathrm{~cm})\end{array}$ & Zhang et al. [25] & 2017 & $\begin{array}{c}0.1 \mathrm{cc} / \mathrm{min} \\
(\mathrm{ID}=2.452-2.550 \mathrm{~cm})\end{array}$ \\
\hline Torabi et al. [42] & 2015 & $0.2-0.4 \mathrm{cc} / \mathrm{min}$ & & & \\
\hline
\end{tabular}

\section{CONCLUSIONS}

The preceding review of previous studies shows that in spite of numerous investigations

3 spanning over half a century, the issue of temperature's impact on oil-water relative permeability

4 is still not fully resolved. New findings are still being reported on this topic [117]. There appear to

5 be three reasons for the lack of consensus in experimentally observed results:

6 (1) The measurements of relative permeability at high temperature are complex and often the

$7 \quad$ reported results include experimental artifacts.

8 (2) Meaningful relative permeability measurements require that capillary forces control the

9 fluid distribution within the pore space, but this condition is difficult to ensure in viscous

$10 \quad$ oil systems.

11 (3) The impact of temperature is not same in all rock-fluid systems, it depends on how the

12 wettability, interfacial tension and sometimes even the pore geometry changes with 13 temperature. 
1 In reservoir systems that have a higher potential for temperature effect, it would be advisable

2 to obtain experimental measurements. These should include not only relative permeability tests

3 but also measurements of wettability and interfacial tensions. Without such experimental

4 measurements, it might be just as good to assume that the relative permeability is independent of

5 temperature as to assume that it will move with temperature in a predictable manner.

\section{ACKNOWLEDGEMENT}

The financial support for this work was provided by NSERC/Nexen and CNOOC Industrial

8 Research Chair in Advanced In-Situ Recovery Processes for Oil Sands program and University of

9 Calgary's Global Research Initiative in Sustainable Low Carbon Unconventional Resources, 10 funded from the Canada First Research Excellence Fund.

\section{NOMENCLATURE}

$\begin{array}{llll}C^{*} & \text { Wettability number } & D & \text { Core diameter } \\ g & \text { Gravitational acceleration } & k_{r o} & \text { Relative permeability to oil } \\ k_{w} & \text { Absolute permeability to water } & k_{r i} & \text { Relative permeability to it }{ }^{\text {th }} \text { phase } \\ k_{\text {wor }} & \text { Water relative permeability endpoint } & k_{a b s} & \text { Absolute permeability } \\ k_{e i} & \text { Effective permeability to it } \text { in }^{\text {th }} \text { phase } & k_{r o}^{0} & \text { Oil relative permeability endpoint } \\ k_{o i r} & \text { Oil relative permeability endpoint } & k_{r w}^{0} & \text { Water relative permeability endpoint } \\ L & \text { Core length } & M & \text { Mobility ratio } \\ N_{c} & \text { Capillary number } & n w & \text { Water exponent } \\ n o & \text { Oil exponent } & S_{w} & \text { Water saturation } \\ S_{o r} & \text { Residual oil saturation } & S_{e} & \text { Normalized water saturation }\end{array}$




$\begin{array}{llcc}S_{w i} & \text { Irreducible water saturation } & S_{i} & \mathrm{i}^{\text {th }} \text { phase saturation } \\ T & \text { Temperature } & V & \text { Fluid velocity }\end{array}$

\section{Greek symbols}

$\begin{array}{llll}\alpha & \text { Angle core makes with the vertical } & \mu & \text { Displacing fluid viscosity } \\ \mu_{w} & \text { Water viscosity } & \mu_{o} & \text { Oil viscosity } \\ v^{*} & \text { Constant superficial velocity } & v & \text { Fluid velocity } \\ v_{c} & \text { Characteristic velocity } & \rho_{o} & \text { Oil density } \\ \rho_{w} & \text { Water density } & \sigma & \text { Interfacial tension } \\ \theta & \text { Contact angle } & & \end{array}$

\section{Abbreviation}

$\begin{array}{llll}\text { CA } & \text { Cross section area } & \text { CSS } & \text { Cyclic steam stimulation } \\ \text { ID } & \text { Inner diameter } & \text { IFT } & \text { Interfacial tension } \\ \text { N/A } & \text { Information not available } & \text { PV } & \text { Pore volume } \\ \text { ROS } & \text { Residual oil saturation } & \text { SAGD } & \text { Steam assisted gravity drainage }\end{array}$

SARA Saturate-Aromatic-Resin-Asphaltene

\section{REFERENCES}

4 [1] Ramey H. In Situ Combustion. 8th World Petroleum Congress. World Petroleum 5 Congress; 1971.

6 [2] Akhlaghinia M, Torabi F, Chan CW. Effect of temperature on two-phase relative 7 permeabilities of heavy oil, water, carbon dioxide, and methane determined by 8 displacement technique. Energy \& Fuels 2013;27(3):1185-93.

9 [3] Ashrafi M, Souraki Y, Torsaeter O. Effect of Temperature on Athabasca Type Heavy OilWater Relative Permeability Curves in Glass Bead Packs. Energy and Environment Research 2012;2(2):113. 
[4] Ashrafi M, Souraki Y, Torsaeter O. Investigating the Temperature Dependency of Oil and Water Relative Permeabilities for Heavy Oil Systems. Transport in porous media 2014;105(3):517-37.

[5] Mosavat N, Mohsenzadeh A, Al-Wahaibi Y. Estimating Oil/Water Relative Permeability at SAGD Steam Chamber Edge. SPE Heavy Oil Conference and Exhibition. Society of Petroleum Engineers; 2016.

[6] Polikar M, Ali S, Puttagunta V. High-temperature relative permeabilities for Athabasca oil sands. SPE Reservoir Engineering 1990;5(01):25-32.

[7] Polikar M, Ferracuti F, Decastro V, Puttagunta R, Ali S. Effect of temperature on bitumenwater end point relative permeabilities and saturations. Journal of Canadian Petroleum Technology 1986;25(05).

[8] Maini BB, Okazawa T. Effects of temperature on heavy oil-water relative permeability of sand. Journal of Canadian Petroleum Technology 1987;26(03).

[9] Nazari J, Nasiry F, Seddiqi N, Honma S. Influence of relative permeability and viscosity ratio on oil displacement by water in petroleum reservoir. Proceedings of School of Engineering of Tokai University 2015;40:16-8.

[10] Odeh AS. Effect of Viscosity Ratio on Relative Permeability (includes associated paper 1496-G). 1959.

[11] Wang J, Dong M, Asghari K. Effect of Oil Viscosity on Heavy Oil-Water Relative Permeability Curves. SPE/DOE Symposium on Improved Oil Recovery. Society of Petroleum Engineers; 2006.

[12] Esfahani MR, Haghighi M. Wettability evaluation of Iranian carbonate formations. Journal of Petroleum Science and Engineering 2004;42(2):257-65.

[13] Hamouda AA, Karoussi O. Effect of temperature, wettability and relative permeability on oil recovery from oil-wet chalk. Energies 2008;1(1):19-34.

[14] Lo HY, Mungan N. Effect of temperature on water-oil relative permeabilities in oil-wet and water-wet systems. Fall Meeting of the Society of Petroleum Engineers of AIME. Society of Petroleum Engineers; 1973.

[15] Maini B, Batycky J. Effect of temperature on heavy-oil/water relative permeabilities in horizontally and vertically drilled core plugs. Journal of petroleum technology 1985;37(08):1,500-1,10.

[16] Poston S, Ysrael S, Hossain A, Montgomery III E. The effect of temperature on irreducible water saturation and relative permeability of unconsolidated sands. Society of Petroleum Engineers Journal 1970;10(02):171-80.

[17] Sola BS, Rashidi F, Babadagli T. Temperature effects on the heavy oil/water relative permeabilities of carbonate rocks. Journal of petroleum science and engineering 2007;59(1):27-42.

[18] Vega B, Anthony Robert K. Steady-state relative permeability measurements, temperature dependency and a reservoir diatomite core sample evolution. SPE Annual Technical Conference and Exhibition. Society of Petroleum Engineers; 2014.

[19] Watson R, Ertekin T. The effect of steep temperature gradient on relative permeability measurements. SPE Rocky Mountain Regional Meeting. Society of Petroleum Engineers; 1988.

[20] Weinbrandt R, Ramey Jr H, Casse F. The effect of temperature on relative and absolute permeability of sandstones. Society of Petroleum Engineers Journal 1975;15(05):376-84 . 
[21] Edmondson T. Effect of temperature on waterflooding. Journal of Canadian Petroleum Technology 1965;4(04):236-42.

[22] Hamouda A, Karoussi O, Chukwudeme E. Relative permeability as a function of temperature, initial water saturation and flooding fluid compositions for modified oil-wet chalk. Journal of Petroleum Science and Engineering 2008;63(1):61-72.

[23] Sinnokrot AA, Ramey Jr HJ, Marsden Jr S. Effect of temperature level upon capillary pressure curves. Society of Petroleum Engineers Journal 1971;11(01):13-22.

[24] Torabzadeh S, L.L. Handy. The effect of temperature and interfacial tension on water/oil relative permeabilities of consolidated sands. SPE Enhanced Oil Recovery Symposium. Society of Petroleum Engineers; 1984.

[25] Zhang L-h, Tong J, Xiong Y, Zhao Y-l. Effect of temperature on the oil-water relative permeability for sandstone reservoirs. International Journal of Heat and Mass Transfer 2017;105:535-48.

[26] Maini B. Is it futile to measure relative permeability for heavy oil reservoirs? Journal of Canadian Petroleum Technology 1998;37(04).

[27] Ahmed T. Reservoir engineering handbook. Elsevier; 2006.

[28] Dake LP. The practice of reservoir engineering (revised edition). Elsevier; 2001.

[29] Honarpour MM, Koederitz F, Herbert A. Relative permeability of petroleum reservoirs. 1986.

[30] Du Yuqi OB, Dacun L. Literature review on methods to obtain relative permeability data. 2004.

[31] Maini B, Coskuner G, Jha K. A comparison of steady-state and unsteady-state relative permeabilities of viscocities oil and water in ottawa sand. Journal of canadian petroleum technology 1990;29(02).

[32] Anderson WG. Wettability literature survey-part 6: the effects of wettability on waterflooding. Journal of petroleum technology 1987;39(12):1,605-1,22.

[33] Buckley SE, Leverett M. Mechanism of fluid displacement in sands. Transactions of the AIME 1942;146(01):107-16.

[34] Leverett MC. Flow of oil-water mixtures through unconsolidated sands. Transactions of the AIME 1939;132(01):149-71.

[35] Anderson W. Wettability literature survey-part 2: Wettability measurement. Journal of petroleum technology 1986;38(11):1,246-1,62.

[36] Glover P. Formation Evaluation MSC course notes. Aberdeen University 2001:84-94.

[37] Akin S, Castanier LM, Brigham WE. Effect of temperature on heavy-oil/water relative permeabilities. SPE Annual Technical Conference and Exhibition. Society of Petroleum Engineers; 1998.

[38] Bennion D, Thomas F, Schulmeister B, Ma T. A Correlation of the Low and High Temperature Water-Oil Relative Permeability Characteristics of Typical Western Canadian Unconsolidated Bitumen Producing Formations. Canadian International Petroleum Conference. Petroleum Society of Canada; 2006.

[39] Esmaeili S, Sarma H, Harding T, Maini B. A data-driven model for predicting the effect of temperature on oil-water relative permeability. Fuel 2019;236:264-77.

[40] Kumar S, Torabzadeh S, Handy L. Relative permeability functions for high-and lowtension systems at elevated temperatures. SPE California Regional Meeting. Society of Petroleum Engineers; 1985. 
[41] Nakornthap K, Evans RD. Temperature-dependent relative permeability and its effect on oil displacement by thermal methods. SPE Reservoir Engineering 1986;1(03):230-42.

[42] Torabi F, Mosavat N, Zarivnyy O. Predicting heavy oil/water relative permeability using modified Corey-based correlations. Fuel 2016;163:196-204.

[43] Abasov M, Tairov N, Abdullaeva A, Alieva S, Mamedov A. Influence of temperature on relative phase permeability at high pressures. Dokl. Akad. Nauk Azerb. SSR. 8. 1976:31-4.

[44] Bennion D, Moore R, Thomas F. Effect of relative permeability on the numerical simulation of the steam stimulation process. Journal of Canadian Petroleum Technology 1985;24(02).

[45] Cao L, Li S. The Effect of Temperature and Rock Permeability on Oil-Water Relative Permeability Curves of Waxy Crude Oil. International Journal of Engineering Research and Applications 2016;6(4):16-21.

[46] Frizzell D. Analysis of 15 years of thermal laboratory data: Relative permeability and saturation endpoint correlations for heavy oils. SPE Annual Technical Conference and Exhibition. Society of Petroleum Engineers; 1990.

[47] Kumar M, Inouye T. Low-temperature analogs of high-temperature water/oil relative permeabilities. SPE Annual Technical Conference and Exhibition. Society of Petroleum Engineers; 1994.

[48] Nourmohammad A, Vahidi A, Emadi M, Gerami S. Effect of Temperature on Two Phase Oil-Water Relative Permeabilities. 77th EAGE Conference and Exhibition 2015. 2015.

[49] Schembre J, Tang G-Q, Kovscek A. Wettability alteration and oil recovery by water imbibition at elevated temperatures. Journal of Petroleum Science and Engineering 2006;52(1):131-48.

[50] Schembre JM, Tang G-q, Kovscek AR. Effect of temperature on relative permeability for heavy-oil diatomite reservoirs. SPE Western Regional Meeting. Society of Petroleum Engineers; 2005.

[51] Sufi AH, Ramey Jr HJ, Brigham WE. Temperature effects on relative permeabilities of oilwater systems. SPE Annual Technical Conference and Exhibition. Society of Petroleum Engineers; 1982.

[52] Wang Y, Wang S, Jiang Z. The influence of temperature and interfacial tension on oilwater relative permeability. Oil Gasfield Surface Engineering 2004;23(4):11-2.

[53] Zeidani M, Maini B. SAGD Relative Permeability as a Function of Temperature. SPE Canada Heavy Oil Technical Conference. Society of Petroleum Engineers; 2016.

[54] Brown HW. Capillary pressure investigations. Journal of Petroleum Technology 1951;3(03):67-74.

[55] McCaffery FG. The effect of wettability on relative permeability and imbibition in porous media. 1973.

[56] Anderson WG. Wettability literature survey part 5: the effects of wettability on relative permeability. Journal of Petroleum Technology 1987;39(11):1,453-1,68.

[57] Donaldson EC, Crocker ME. Review of petroleum oil saturation and its determination. HEDB (Historical Energy Database (United States)); 1977.

[58] Donaldson EC, Thomas RD. Microscopic observations of oil displacement in water-wet and oil-wet systems. Fall Meeting of the Society of Petroleum Engineers of AIME. Society of Petroleum Engineers; 1971.

[59] Moore T, Slobod R. The effect of viscosity and capillarity on the displacement of oil by water. Producers Monthly 1956;20(10):20-30. 
[60] Mattax C, Kyte J. Ever see a water flood. Oil Gas J 1961;59(42):115-28.

2 [61] Honarpour M, Mahmood S. Relative-permeability measurements: An overview. Journal of 3

[62] Virnovsky G, Skjaeveland S, Surdal J, Ingsoy P. Steady-state relative permeability measurements corrected for capillary effects. SPE Annual Technical Conference and Exhibition. Society of Petroleum Engineers; 1995.

[63] Maini B, Kokal S, Jha K. Measurements and correlations of three-phase relative permeability at elevated temperatures and pressures. SPE Annual Technical Conference and Exhibition. Society of Petroleum Engineers; 1989.

[64] Muqeem M, Bentsen R, Maini B. Effect of Temperature On Th. ree-phase Water-oil-gas Relative Permeabilities of UnconsoUdated Sand. Journal of Canadian Petroleum Technology 1995;34(03).

[65] Caudle B, Slobod R, Brownscombe E. Further developments in the laboratory determination of relative permeability. Journal of Petroleum Technology 1951;3(05):14550 .

[66] Morse R. Relative permeability measurements on small core samples. Mineral Industries Experiment Station; 1947.

[67] Osoba J, Richardson J, Kerver J, Hafford J, Blair P. Laboratory measurements of relative permeability. Journal of Petroleum Technology 1951;3(02):47-56.

[68] Josendal V, Sandiford B, Wilson J. Improved multiphase flow studies employing radioactive tracers. Journal of Petroleum Technology 1952;4(03):65-76.

[69] Loomis AG, Crowell DC. Relative permeability studies: gas-oil and water-oil systems. Bureau of Mines, San Francisco, Calif.(USA). San Francisco Petroleum Research Lab.; 1961.

[70] Richardson J, Kerver J, Hafford J, Osoba J. Laboratory determination of relative permeability. Journal of Petroleum Technology 1952;4(08):187-96.

[71] Leas W, Jenks J, Russell CD. Relative permeability to gas. Journal of Petroleum Technology 1950;2(03):65-72.

[72] Hassler GL. Method and apparatus for permeability measurements. Google Patents; 1944.

[73] Rose W. Some problems in applying the Hassler relative permeability method. Journal of Petroleum Technology 1980;32(07):1,161-1,3.

[74] Saraf DN, McCaffery FG. Two-and Three-phase Relative Permeabilities: A Review. Petroleum Recovery Institute; 1981.

[75] Burdine N. Relative permeability calculations from pore size distribution data. Journal of Petroleum Technology 1953;5(03):71-8.

[76] Fatt I, Dykstra H. Relative permeability studies. Journal of Petroleum Technology 1951;3(09):249-56.

[77] Purcell W. Capillary pressures-their measurement using mercury and the calculation of permeability therefrom. Journal of Petroleum Technology 1949;1(02):39-48.

[78] Timmerman E. Practical reservoir engineering. PennWell Corporation; 1982.

[79] Wylie M, Gardner G. The generalized Kozeny-Carman equation: Its application to problems of multiphase flow in porous media. World Oil 1958;146:210-27.

[80] Firoozabadi A, Aziz K. Relative permeabilities from centrifuge data. Journal of Canadian Petroleum Technology 1991;30(05). 
[81] O'Meara Jr D, Lease W. Multiphase relative permeability measurements using an automated centrifuge. SPE Annual Technical Conference and Exhibition. Society of Petroleum Engineers; 1983.

[82] Davidson L. The effect of temperature on the permeability ratio of different fluid pairs in two-phase systems. Journal of Petroleum Technology 1969;21(08):1,037-1,46.

[83] Craig FF. The reservoir engineering aspects of waterflooding. HL Doherty Memorial Fund of AIME New York; 1971.

[84] Closmann P, Waxman M, Deeds C. Steady-state tar/water relative permeabilities in Peace River cores at elevated temperature. SPE Reservoir Engineering 1988;3(01):76-80.

[85] Farasat A, Shokrollahi A, Arabloo M, Gharagheizi F, Mohammadi AH. Toward an intelligent approach for determination of saturation pressure of crude oil. Fuel processing technology 2013;115:201-14.

[86] Wilson J. Determination of relative permeability under simulated reservoir conditions. AIChE Journal 1956;2(1):94-100.

[87] Ehrlich R. The effect of temperature on water-oil imbibition relative permeability. SPE Eastern Regional Meeting. Society of Petroleum Engineers; 1970.

[88] Lefebvre du Prey E. Factors affecting liquid-liquid relative permeabilities of a consolidated porous medium. Society of Petroleum Engineers Journal 1973;13(01):39-47.

[89] Amaefule JO, Handy LL. The effect of interfacial tensions on relative oil/water permeabilities of consolidated porous media. Society of Petroleum Engineers Journal 1982;22(03):371-81.

[90] Miller MA, Ramey Jr H. Effect of temperature on oil/water relative permeabilities of unconsolidated and consolidated sands. Society of Petroleum Engineers Journal 1985;25(06):945-53.

[91] Hawkins JT. Comparison of three methods of relative permeability measurement. The Log Analyst 1989;30(05).

[92] Siddiqui S, Hicks PJ, Ertekin T. Two-Phase Relative PermeabilityModels in Reservoir Engineering Calculations. Energy sources 1999;21(1-2):145-62.

[93] Karaei MA, Ahmadi A, Fallah H, Dabiri A. Experimental Study of Temperature Effect on Oil Relative Permeability in Porous Media. Defect and Diffusion Forum. 336. Trans Tech Publ; 2013:19-27.

[94] Li B, fen Pu W, xing Li K, Jia H, Wang KY, guang Yang Z. The Characteristics and Impacts Factors of Relative Permeability Curves in High Temperature and LowPermeability Limestone Reservoirs. Advanced Materials Research 2014.

[95] Moss JT. Predicting Oil Displacement by Thermal Methods. SPE California Regional Meeting. Society of Petroleum Engineers; 1981.

[96] Honarpour M, Koederitz L, Harvey AH. Empirical equations for estimating two-phase relative permeability in consolidated rock. Journal of Petroleum Technology 1982;34(12):2,905-2,8.

[97] Adamson AW, Gast AP. Physical chemistry of surfaces. 1967.

[98] Young T. III. An essay on the cohesion of fluids. Philosophical transactions of the royal society of London 1805;95:65-87.

[99] Nicholas MG, Drevet B. Wettability at high temperatures [electronic resource]. Pergamon; 1999.

45 [100] Amott E. Observations relating to the wettability of porous rock. 1959. 
[101] Donaldson EC, Thomas RD, Lorenz PB. Wettability determination and its effect on recovery efficiency. Society of Petroleum Engineers Journal 1969;9(01):13-20.

[102] Fulcher Jr RA, Ertekin T, Stahl C. Effect of capillary number and its constituents on twophase relative permeability curves. Journal of petroleum technology 1985;37(02):249-60.

[103] Brooks RH, Corey AT. Properties of porous media affecting fluid flow. Journal of the Irrigation and Drainage Division 1966;92(2):61-90.

[104] Corey AT. The interrelation between gas and oil relative permeabilities. Producers monthly 1954;19(1):38-41.

[105] Sigmund P, McCaffery F. An improved unsteady-state procedure for determining the relative-permeability characteristics of heterogeneous porous media (includes associated papers 8028 and 8777). Society of Petroleum Engineers Journal 1979;19(01):15-28.

[106] Chierici GL. Novel relations for drainage and imbibition relative permeabilities. Society of Petroleum Engineers Journal 1984;24(03):275-6.

[107] Lomeland F, Ebeltoft E, Thomas WH. A new versatile relative permeability correlation. International Symposium of the Society of Core Analysts, Toronto, Canada. 112. 2005.

[108] Jones S, Roszelle W. Graphical techniques for determining relative permeability from displacement experiments. Journal of Petroleum Technology 1978;30(05):807-17.

[109] Gomari KR, Hamouda A. Effect of fatty acids, water composition and pH on the wettability alteration of calcite surface. Journal of petroleum science and engineering 2006;50(2):14050.

[110] Karoussi O, Hamouda AA. Imbibition of sulfate and magnesium ions into carbonate rocks at elevated temperatures and their influence on wettability alteration and oil recovery. Energy \& fuels 2007;21(4):2138-46.

[111] Akhlaghinia M, Torabi F, Chan CW. Experimental investigation of temperature effect on three-phase relative permeability isoperms in heavy oil systems. Fuel 2014;118:281-90.

[112] Johnson E, Bossler D, Bossler V. Calculation of relative permeability from displacement experiments. 1959.

[113] Rapoport L, Leas W. Properties of linear waterfloods. Journal of Petroleum Technology 1953;5(05):139-48.

[114] Peters EJ, Flock DL. The onset of instability during two-phase immiscible displacement in porous media. Society of Petroleum Engineers Journal 1981;21(02):249-58.

[115] de Haan J. Effect of Capillary Forces in the Water-Drive Process. 5th World Petroleum Congress. World Petroleum Congress; 1959.

[116] Flock D, Peters E, Baird H, Wilborg R, Kloepfer J. The Influence of Frontal Instabilities During Viscous Oil Displacement. The Oil Sands of Canada-Venezuela 1977;1101(17):380-5.

[117] Qin Y, Wu Y, Liu P, Zhao F, Yuan Z. Experimental studies on effects of temperature on oil and water relative permeability in heavy-oil reservoirs. Scientific Reports 2018;8(1):12530. 